\title{
Active and Multi-View Machine Learning for microRNA Prediction
}

\author{
By \\ Mohsen Sheikh Hassani
}

A thesis submitted to the Faculty of Graduate Studies and Research in partial fulfillment of the requirements for the degree of

Masters of Applied Science

in Biomedical Engineering

Ottawa-Carleton Institute for Biomedical Engineering

Department of Systems and Computer Engineering

Carleton University

Ottawa, Ontario, Canada

August 2018 


\section{Abstract}

MicroRNA (miRNA) are short, non-coding RNAs involved in cell regulation at posttranscriptional and translational levels. MiRNA act on messenger RNA through a silencing mechanism, affecting biological activities such as cell cycle control, biological development, differentiation, and stress response. Experimental validation of predicted miRNA is a time and cost expensive procedure, as it requires wet-lab experiments. Therefore, a variety of computational approaches have been developed to increase prediction accuracy and reduce validation costs. While these methods are highly effective, they require large labelled training data sets, which are often not available for many species. Simultaneously, emerging wet-lab experimental procedures are becoming available that produce large unlabelled data sets of genomic sequence and RNA expression profiles. Existing methods are unable to leverage these unlabelled data.

This thesis explores two emerging trends in semi-supervised machine learning to maximize the utility of both labelled and unlabelled training data. Specifically, this thesis explores the application of active learning and multi-view co-training to microRNA prediction for the first time. Results show that our active learning approach is able to greatly improve classification performance using a small number of labeled instances, outperforming state-of-the-art methods under equivalent training data constraints. Multi-view co-training results also demonstrate improved performance compared to single view classifiers and yield high classification performance using a minimum number of labeled instances for classification. This thesis demonstrates that semi-supervised machine learning is likely be useful in creating predictors of novel miRNA, particularly for species where few training exemplars are available. 


\section{Acknowledgements}

I would like to dedicate this thesis to my father and mother, whom I would not be where I am today without them. They have always been my motivation and role models in life, and their never-ending, unconditional love and support is what has inspired me to move forward and try to make them proud.

I would like to thank my supervisor Dr. James Green for his patience, guidance and continuous support from the very first day. I consider myself lucky to have been able to work under the supervision of such an amazing professor.

A special thank you to my caring sister Masoomeh, as well as Saeed for their help and support during these two years. I would also like to thank all friends and members of my family for their never-ending love and support.

This study was supported by the Natural Sciences and Engineering Research Council of Canada. 


\section{Table of Contents}

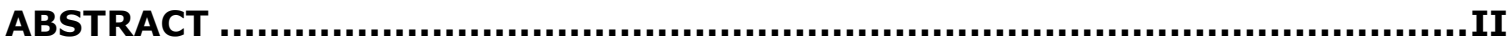

ACKNOWLEDGEMENTS.......................................................................... III

TABLE OF CONTENTS ............................................................................. IV

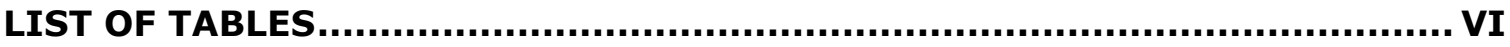

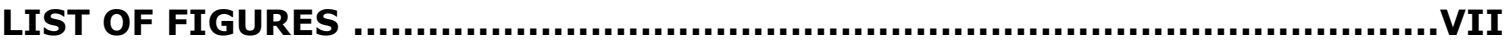

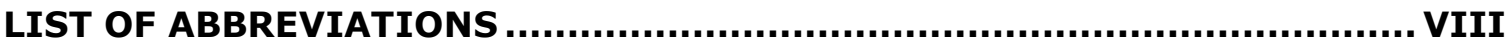

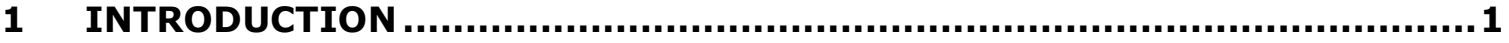

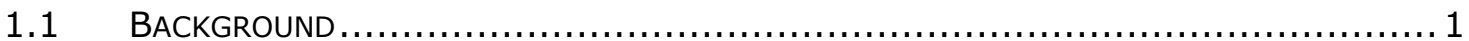

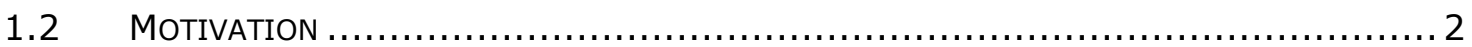

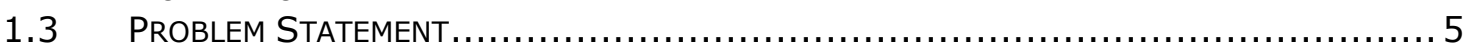

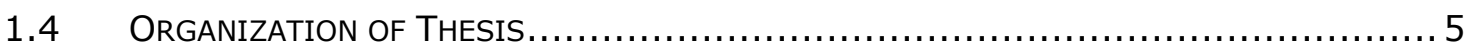

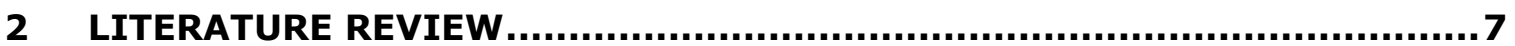

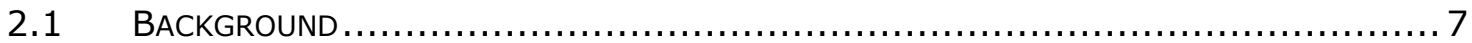

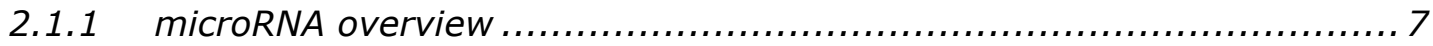

2.1.2 Pattern Classification ..................................................... 10

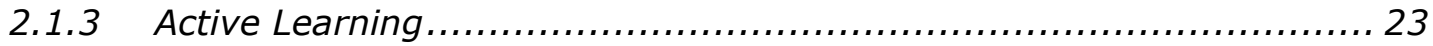

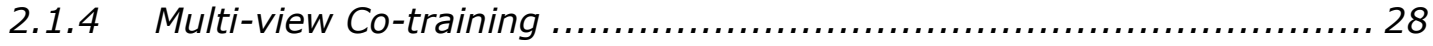

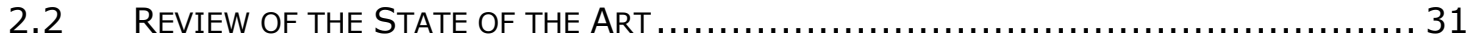

2.2.1 Prediction of miRNA....................................................... 31

2.2.2 Previous applications of co-training to machine learning .................. 38

2.2.3 Active learning in bioinformatics ............................................ 44

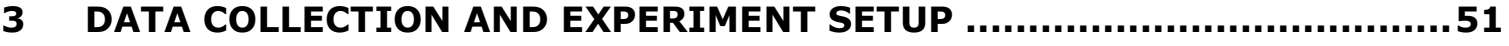

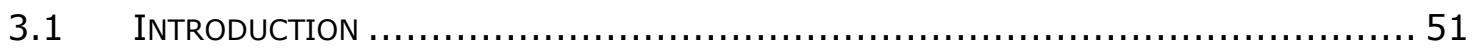

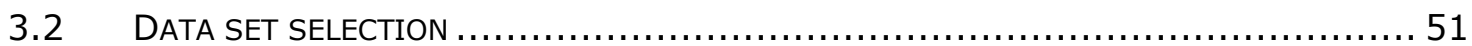

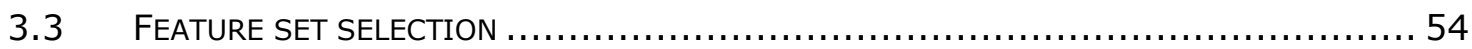

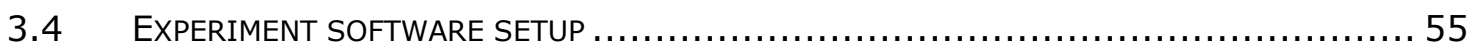

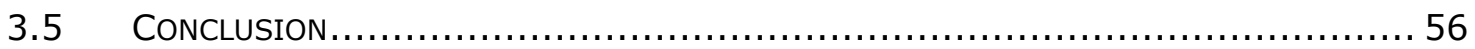

4 ACTIVE LEARNING FOR MIRNA PREDICTION ................................ 57

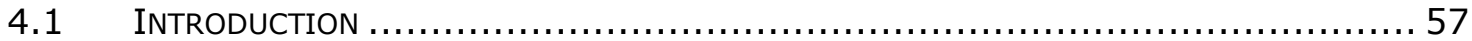

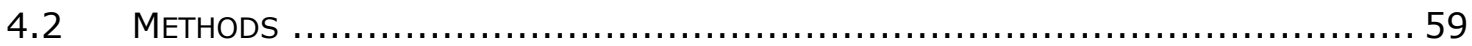

4.2.1 Selecting Size of Initial Seed Training Set ............................... 61

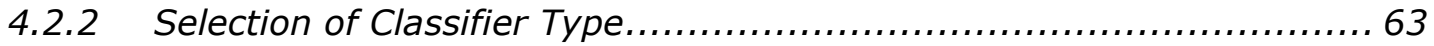

4.2.3 Selecting Stopping Criterion .................................................... 64

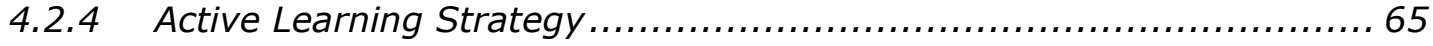

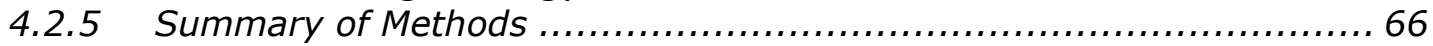

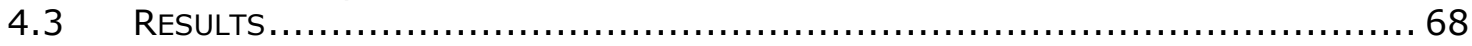

4.3.1 Comparing Uncertainty- and Certainty-based Active Learning ............68 68

4.3.2 Passive vs. Active Learning ............................................... 72

4.3.3 Benefits of an Integrated Feature Set .................................... 76

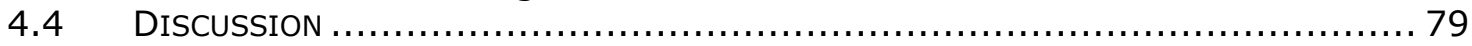

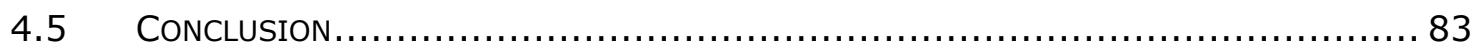


5 MULTI-VIEW CO-TRAINING FOR MIRNA PREDICTION $\ldots \ldots \ldots \ldots \ldots \ldots \ldots \ldots . . . . . . \ldots 4$

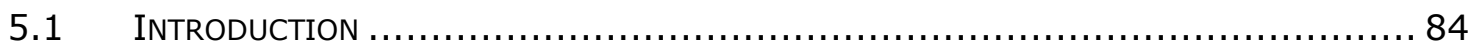

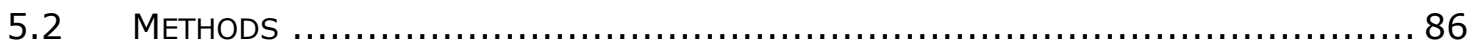

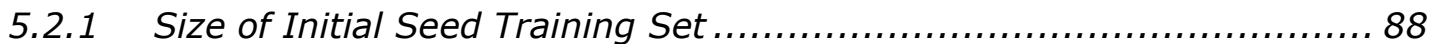

5.2 .2 Feature Selection ......................................................... 89

5.2.3 Classifier Model Selection ................................................ 90

5.2.4 Co-Training Stopping Criterion........................................... 92

5.2.5 Summary of Co-Training Approach ...................................... 93

5.2.6 Benchmark Algorithms....................................................... 94

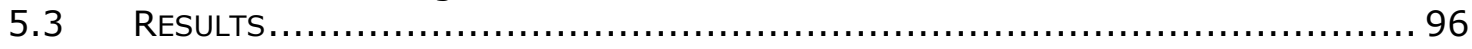

5.3.1 Establishing the Effectiveness of Co-Training for miRNA Prediction ..... 96

5.3.2 Comparing Co-Training with Benchmark Methods....................... 100

5.3.3 Combining Views for Consensus Decision ............................. 105

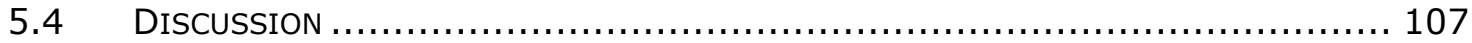

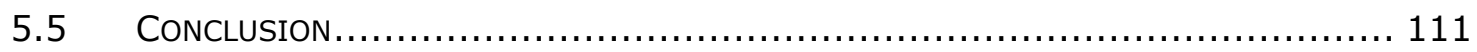

6 THESIS SUMMARY AND FUTURE RECOMMENDATIONS ....................112

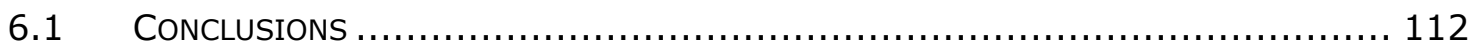

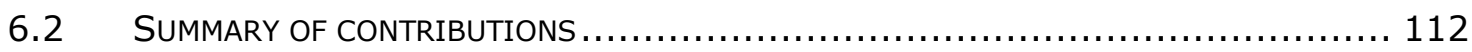

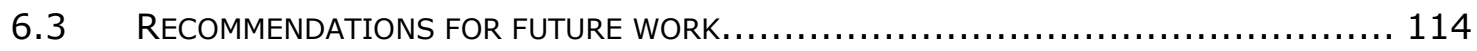

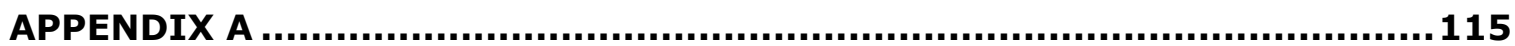

REFERENCES...................................................................................... 121 


\section{List of Tables}

TABLE 1. LIST OF DATASETS USED IN EXPERIMENTS. 52

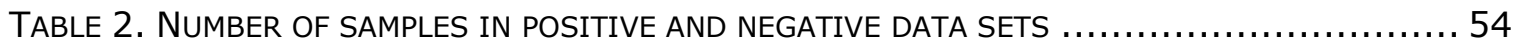

TABLE 3. AVERAGE AREA UNDER ROC AND PRECISION-RECALL CURVES FOR CLASSIFIER USING DIFFERENT TRAINING SET SIZES

TABLE 4. COMPARISON OF THE AREA UNDER PRECISION-RECALL FOR ACTIVE LEARNING USING SVM AND RANDOM FOREST CLASSIFIERS ON HSA DATA SET. MEANS \pm STANDARD DEVIATIONS ARE SHOWN, REPRESENTING 100 REPETITIONS OF EACH EXPERIMENT $\ldots \ldots \ldots \ldots \ldots \ldots \ldots \ldots \ldots \ldots \ldots$

TABLE 5. CERTAINTY AND UNCERTAINTY-BASED ACTIVE LEARNING QUERY STRATEGY RESULTS FOR HUMAN DATA SET. VALUES REPRESENT THE MEAN ( \pm STANDARD DEVIATION) OF 100

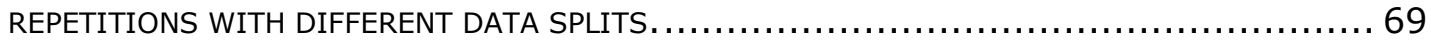

TABLE 6. FINAL CLASSIFICATION RESULTS USING CERTAINTY AND UNCERTAINTY-BASED ACTIVE LEARNING STRATEGIES, PASSIVE TRAINING, SELF-LEARNING AND "NO LEARNING" FOR ALL SIX

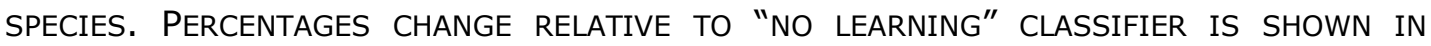

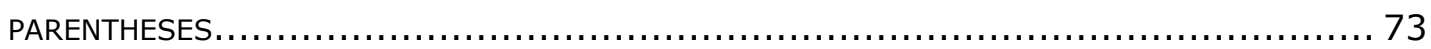

TABLE 7. COMPARING AVERAGE AUPRC FOR ALL SIX DATA SETS OVER THE FOLLOWING METHODS: ACTIVE LEARNING WITH INTEGRATED FEATURE SET, PASSIVE SEQUENCE-BASED ONLY CLASSIFIER, PASSIVE EXPRESSION-BASED ONLY CLASSIFIER, PASSIVE INTEGRATED CLASSIFIER, AND MIRDEEP2. MEANS \pm STANDARD DEVIATIONS ARE SHOWN, REPRESENTING 100

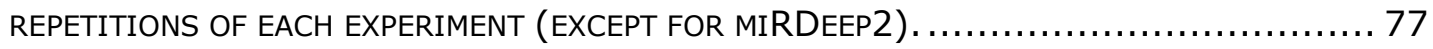

TABLE 8. AREA UNDER PRECISION-RECALL CURVE FOR SEQUENCE- AND EXPRESSION-BASED

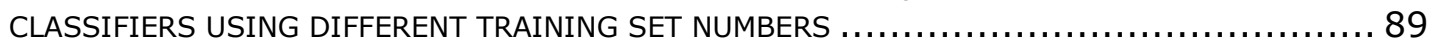

TABLE 9. PERFORMANCE OF SEQUENCE AND EXPRESSION-BASED VIEWS FOR RF AND SVM OVER 6 ITERATIONS OF CO-TRAINING ON THE HUMAN DATASET. MEANS \pm STANDARD DEVIATIONS ARE SHOWN, REPRESENTING 100 REPETITIONS OF EACH EXPERIMENT. 91

TABLE 10. CO-TRAINING PERFORMANCE RESULTS FOR HUMAN DATA SET. MEANS \pm STANDARD DEVIATIONS ARE SHOWN, REPRESENTING 100 REPETITIONS OF EACH EXPERIMENT.

TABLE 11. FINAL CLASSIFICATION RESULTS USING CO-TRAINING, SINGLE-VIEW (SELF-TRAINING), PASSIVE LEARNING AND NO LEARNING FOR EXPRESSION AND SEQUENCE-BASED VIEWS...... 101 


\section{List of Figures}

Figure 1. GenERAL STRUCTURE OF A MiRNA (ADAPTED FROM [8]) $\ldots \ldots \ldots \ldots \ldots \ldots \ldots \ldots \ldots \ldots \ldots$

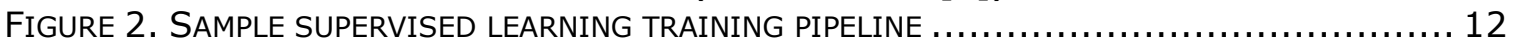

FIGURE 3. SAMPLE DECISION TREE FOR FRUIT CLASSIFICATION (ADAPTED FROM [29]) ............ 15

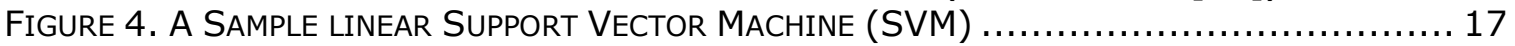

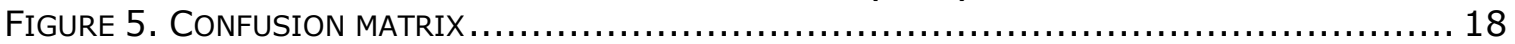

FIGURE 6. SAMPLE ROC CURVE WITH DIFFERENT CLASSIFIER PERFORMANCE DEMONSTRATION (ADAPTED FROM [34]). CURVE A REPRESENTS A PERFECT CLASSIFIER, CURVE B AND C REPRESENT REAL-WORLD CLASSIFIERS, WHERE THE PERFORMANCE OF CLASSIFIER B EXCEEDS CLASSIFIER C, AND CURVE D REPRESENTS A RANDOM CLASSIFIER $\ldots \ldots \ldots \ldots \ldots \ldots \ldots \ldots \ldots \ldots \ldots \ldots \ldots \ldots \ldots \ldots$

FIGURE 7. SAMPLE PRECISION RECALL CURVE WITH DIFFERENT CLASSIFIER PERFORMANCE DEMONSTRATION. CURVE A REPRESENTS A PERFECT CLASSIFIER, CURVE B AND C REPRESENT REAL-WORLD CLASSIFIERS, WHERE THE PERFORMANCE OF CLASSIFIER B EXCELS CLASSIFIER C,

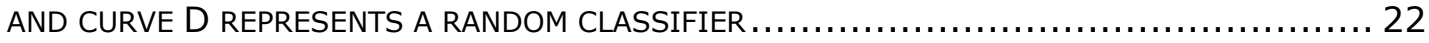

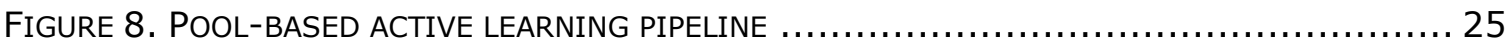

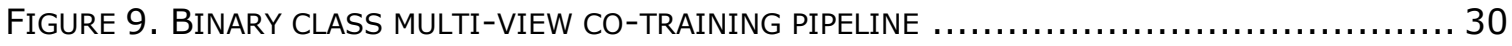

FIGURE 10. INFORMATION PROVIDED BY NGS EXPERIMENT REGARDING A MIRNA (REPRODUCED

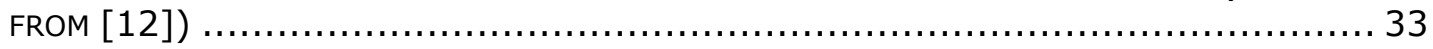

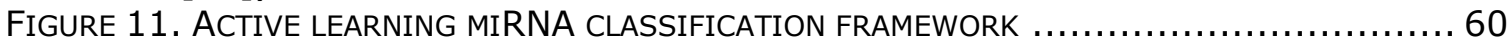

FIGURE 12. AVERAGE PRECISION-RECALL CURVE FOR HUMAN DATA SET AT SPECIFIC ITERATIONS. THE

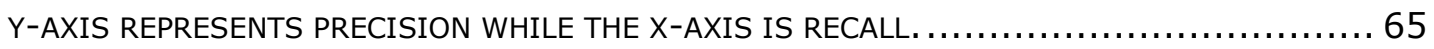

FIGURE 13. CERTAINTY AND UNCERTAINTY-BASED ACTIVE LEARNING QUERY STRATEGY RESULTS VS. PASSIVE AND SELF-LEARNING FOR ALL SIX DATA SETS OVER 11 ITERATIONS. ............... 70

FiguRE 14. PRECISION-RECALL CURVES FOR ALL SIX SPECIES AFTER COMPLETING CERTAINTY AND UNCERTAINTY-BASED ACTIVE LEARNING, PASSIVE LEARNING, SELF-TRAINING AND NO LEARNING. IN ALL PLOTS, THE Y-AXIS REPRESENTS PRECISION WHILE THE X-AXIS IS RECALL. 75

FIGURE 15. COMPARING AVERAGE PRECISION-RECALL CURVES FOR ALL SIX DATA SETS OVER THE FOLLOWING METHODS; ACTIVE LEARNING, SEQUENCE-BASED ONLY CLASSIFIER, EXPRESSIONBASED ONLY CLASSIFIER, INTEGRATED CLASSIFIER, MIRDEEP2. IN ALL PLOTS, THE Y-AXIS

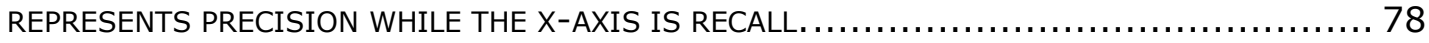

FIGURE 16. CO-TRAINING CLASSIFIER FRAMEWORK REPEATED UNTIL STOPPING CRITERION $\ldots \ldots \ldots . . .87$

FIGURE 17. AVERAGE PRECISION-RECALL CURVE FOR HUMAN DATA SET AT SPECIFIC ITERATIONS FOR EXPRESSION (LEFT FIGURE) AND SEQUENCE-BASED (RIGHT FIGURE) CLASSIFIERS. THE Y-AXIS REPRESENTS PRECISION WHILE THE X-AXIS IS RECALL.................................... 93

Figure 18. PERFORMANCE OF THE SEQUENCE AND EXPRESSION-BASED CLASSIFIERS AT EACH

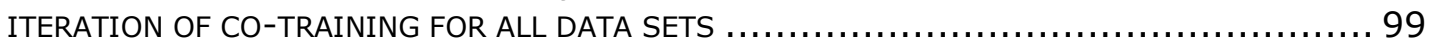

FIGURE 19. FINAL LEARNING CURVES FOR CLASSIFICATION USING CO-TRAINING, SINGLE-VIEW (SELF-TRAINING), PASSIVE LEARNING AND NO LEARNING FOR EXPRESSION AND SEQUENCEBASED VIEWS ON THE HUMAN, MOUSE AND FRUIT-FLY DATA SETS. IN ALL PLOTS, THE Y-AXIS REPRESENTS PRECISION WHILE THE X-AXIS IS RECALL. OPTIMAL PERFORMANCE IS IN THE TOP-

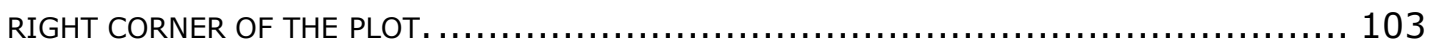

FIGURE 20. FINAL LEARNING CURVES FOR CLASSIFICATION USING CO-TRAINING, SINGLE-VIEW (SELF-TRAINING), PASSIVE LEARNING AND NO LEARNING FOR EXPRESSION AND SEQUENCEBASED VIEWS ON THE COW, CHICKEN AND HORSE DATA SETS. IN ALL PLOTS, THE Y-AXIS REPRESENTS PRECISION WHILE THE X-AXIS IS RECALL. OPTIMAL PERFORMANCE IS IN THE TOP-

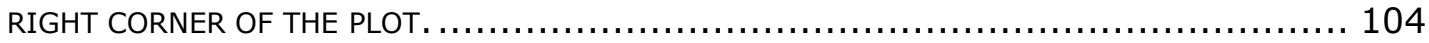

Figure 21. PERFORMANCE OF THE SEQUeNCE AND EXPRESSION-BASED CLASSIFIERS AND THE COMBINED CO-TRAINING CLASSIFIER AT EACH ITERATION FOR ALL DATA SETS $\ldots \ldots \ldots \ldots \ldots \ldots$ 


\section{List of Abbreviations}

\begin{tabular}{|c|c|}
\hline Abbreviation & Definition \\
\hline AUC & Area Under Curve \\
\hline AUPRC & Area Under Precision-Recall curve \\
\hline AUROC & Area Under Received Operating Characteristic Curve \\
\hline bta & Bos taurus (cow) \\
\hline C. elegans & Caenorhabditis elegans (roundworm) \\
\hline dme & Drosophila melanogaster (fruit fly) \\
\hline eca & Equus caballus (horse) \\
\hline FPR & False Positive Rate \\
\hline gga & Gallus gallus (chicken) \\
\hline hsa & Homo sapiens (human) \\
\hline miRISC & miRNA- induced silencing complex \\
\hline miRNA & Micro RNA \\
\hline $\mathrm{mmu}$ & Mus musculus (mouse) \\
\hline mRNA & Messenger RNA \\
\hline NGS & Next Generation Sequencing \\
\hline RNA & Ribonucleic Acid \\
\hline ROC & Received Operating Characteristic \\
\hline S. cerevisiae & Saccharomyces cerevisiae \\
\hline SVM & Support Vector Machines \\
\hline TPR & True Positive Rate \\
\hline
\end{tabular}




\section{Introduction}

\subsection{Background}

Deoxyribonucleic acid (DNA) is a double helix molecule that holds all the genetic information for a species. For this genetic information to be made usable by the cells, DNA is transcribed into a polymeric molecule named Ribonucleic acid (RNA). RNA can convert stored genetic information into proteins that define the cellular functions. MicroRNA (miRNA) are transcribed regions of DNA that, after some biological processes, ultimately turn into short non-coding RNAs typically in the range of 18 to 25 nucleotides. Through experimentation, it has been found that miRNA are involved in cell regulation at post-transcriptional and translational levels through the degradation and translation inhibition of messenger RNA (mRNA). MicroRNA act on mRNA through a silencing mechanism, and target a wide range of mammalian mRNA, affecting biological activities such as cell cycle control [1], biological development [2, 3], differentiation [4] and stress response [5-7]. Considering the significance of all these biological events which miRNA are involved in, miRNA prove to be critical to our understanding and knowledge of biological processes. Therefore, identifying greater numbers of miRNA will aid science in its goal of explaining natural and biological events.

The identification of novel miRNA requires an inter-disciplinary approach, in which both computational and experimental methods are involved. Through experimental approaches, a number of miRNA have already been identified for many distinct species, ranging from more than 2000 known human miRNA, to less than ten known Mallard miRNA [8]. These known miRNA have been used as a basis to create a number of computational methods to predict new miRNA. Such computational methods use machine learning as a tool to identify and abstract the patterns present in previously discovered miRNA. These models seek to identify 
new putative miRNA sequences exhibiting these patterns and classify arbitrary sequence windows as being either miRNA or non-miRNA.

There are generally two different approaches to developing computational predictors of miRNA. Methods that examine the RNA sequence directly are referred to as de novo predictors, while methods that look for evidence of miRNA biogenesis processing within next generation sequencing (NGS) expression data are referred to as NGS- or expression-based methods [9]. These techniques detect pre-miRNA sequences forming miRNA-like hairpins and classify them based on the presence or absence of sequence or expression-based features, respectively.

\subsection{Motivation}

The main challenge in the field of miRNA prediction is the abundance of unlabelled data and the scarcity of labeled examples of miRNA. This makes the task of miRNA classification a very difficult matter, particularly for newly sequenced species where there are few known miRNA exemplars available. According to miRbase [8], of the thousands of existing species, experimentally validated miRNA sequences are available for only 271 species. Approximately $30 \%$ of these species have 15 or fewer known miRNA sequences, meaning that in general most species have very few training exemplars available. The majority of current de novo and NGS-based miRNA prediction techniques use supervised learning methods for the detection of novel miRNA, thereby requiring a large database of known miRNA. In addition, these methods do not always achieve high accuracy, resulting in many sequences being falsely predicted to be miRNA. These false predictions represent $a$ substantial loss of resources, as they lead to unnecessary experimental validation costs. Previous work has demonstrated that relying on training exemplars from other species leads to reduced classification performance for the target species [10]. Therefore, if we are to 
create more effective miRNA predictors, either new miRNA sequences must be identified, or a method must be designed to create reliable miRNA predictors from smaller numbers of known miRNA exemplars.

Obtaining new miRNA examples remains a difficult task, as it requires wet-lab experiments that come at a high time and resource cost. This prompted us to propose two new semisupervised miRNA prediction techniques in this thesis that extract the most information from limited labelled miRNA data, therefore requiring very few known miRNA. We aim to not only minimize the number of labelled training exemplars required, but also to improve the performance of current prediction methods using fewer samples. In this way we can create a more efficient method for the identification of novel miRNA, particularly for species with few known miRNA, while maximizing the return on investment for costly wet-lab validation experiments. As mentioned, all previous methods use supervised machine learning methods, which only make use of labeled instances for classification. Semi-supervised machine learning methods also make use of unlabelled data for classification and are designed to work in situations where we have a small number of known exemplars and a large body of unlabelled data. Such methods aim to learn patterns not only from the few labelled exemplars, but also the unlabelled data when possible and have yet to be applied to the problem of miRNA prediction. We leverage two semi-supervised approaches for this purpose: active learning and multi-view co-training.

In active learning, we assume that we have a small pool of initially labelled training data, a large pool of unlabelled data, and access to an "all-knowing" oracle that can label any exemplar at a cost. In the case of miRNA research, this oracle takes the form of costly wetlab validation experiments. Active learning begins by training an initial predictive model using the small set of labeled training samples. The trained model is then applied to a set of unlabeled instances, predicting the class of each unlabelled instance. Depending on the 
active learning strategy, a number of these instances are selected to be queried for their true class and are added to the training data set. This process is repeated numerous times, resulting in a larger training set and therefore enhanced classification. Using active learning, we aim to utilize a small number of known-miRNA to help us select new instances for annotation. In other words, our active learning strategy aims to spend experimental resources efficiently. In this approach, we iteratively train the best possible miRNA predictor from available training data, then use this predictor to select unlabelled samples for experimental labelling that will provide maximal improvement in classifier prediction accuracy. Different active learning strategies are explored in this thesis to identify the optimal method for selecting new points to label. By selecting the most appropriate active learning strategy, our classifier will query more informative instances, resulting in enhanced performance and reduced cost and annotation effort.

Multi-view co-training makes use of multiple views of a problem to create distinct classifiers, one for each view. Each classifier is trained on a small set of labeled samples and applied to an unlabelled data set. The most confidently predicted unlabelled instances from each of these views are added to the training set without annotation, and this process is repeated multiple times. In multi-view co-training, we take advantage of the fact that the problem of miRNA prediction can be approached from two distinct views: sequence based de novo prediction or expression-based NGS prediction. This creates two views for classification, an expression-based view and a sequence-based view. It should be noted that both of these views have previously been independently applied for miRNA classification and have proven to be effective; however, multi-view co-training has yet to be attempted in the field of miRNA prediction. By applying multi-view co-training, we leverage each view using the other to create more powerful classifiers. The significant advantage of co-training is that there is no need for collecting any new labelled data (i.e. we do not require an oracle). Only a small initial set of labelled miRNA is required; the method uses these data to train two 
views of the problem. The initial training set is expanded by treating unlabeled exemplars confidently predicted by each view as labeled data and continuing this process through multiple iterations. By doing so, co-training fully overcomes the data availability limitation faced by other methods. It also requires no additional wet-lab validation costs, which is another advantage. We seek to demonstrate that leveraging multiple views of the miRNA prediction problem can be used, through multi-view co-training, to create more accuracy predictors without actually labelling more data.

\subsection{Problem Statement}

For the majority of species, a very limited number of known miRNA exists, meaning that we have limited labelled training data. Existing miRNA prediction methods require large training data sets, and therefore are not applicable to such species unless more training data are obtained, which is a costly process. Emerging NGS wet-lab techniques represent an opportunity, in that they can economically produce large unlabelled datasets of genomic and expression profiling data. Existing approaches to miRNA prediction cannot leverage unlabelled data since they employ strictly supervised learning, meaning they can only make use of labeled instances for classification. We therefore explore two semi-supervised machine learning strategies for the first time in the field of miRNA prediction. We use active learning and multi-view co-training in order to make use of easily available unlabelled data for miRNA prediction in different species. Ultimately, we aim to show that semi-supervised learning is an effective way to create miRNA predictors in the case of limited labelled training data.

\subsection{Organization of Thesis}

This thesis consists of 6 chapters. Chapter 2 provides an introduction to miRNA biology, pattern classification methods, and an overview of current state-of-the-art prediction tools. In addition, background information is presented to the reader on machine learning in 
general and a number of algorithms used in this thesis, as well as detailed background information on active learning and co-training algorithms and a review of the current literature. In chapter 3, we describe the experiment setup, including dataset collection and preparation, feature selection and the software framework developed for our work. Chapter 4 describes the active learning framework developed for miRNA classification, including results for different query strategies, and a comparison of the results obtained using our active learning strategy versus different classification strategies. Chapter 5 introduces our multi-view co-training approach for miRNA classification, and a comparison of results using this approach against other methods is presented. Chapter 6 presents a summary of contributions and provides recommendations for future work. 


\section{Literature Review}

This chapter discusses the background concepts present in this thesis and summarizes the relevant literature.

\subsection{Background}

This section will discuss two concepts required prior to discussing the state of the art and the contributions made in this thesis: miRNA prediction and pattern classification. The latter topic will specifically review the semi-supervised techniques of multi-view co-training and active learning.

\subsection{1 microRNA overview}

MicroRNA are short, non-coding RNAs typically in the range of 18 to 25 nucleotides in length. MicroRNA are relatively newly discovered molecules, with the first discovery dating back to 1993 . The next discovery was not made until 7 years later, and ever since there has been an exponential growth in miRNA discovery, with thousands of known miRNA for different species being identified today. Experimental evidence suggests that miRNA play a key role in cell regulation at post-transcriptional and translational levels through degradation of messenger RNA (mRNA) and translation inhibition. The number of miRNA is limited compared to mRNA in most species, with an estimated ratio of 1 miRNA per 30 mRNA in the human genome, however, each miRNA can target hundreds of mRNA. Evidence suggests that approximately $60-90 \%$ of mRNA have the potential to be targeted by miRNA $[11,12]$, ultimately affecting cell cycle control [1], biological development $[2,3]$ and differentiation [4]. Many studies have also found that miRNA act as tumor suppressors in different cancers such as liver [13], colon [14], prostate [15] and bladder cancers [16] to name a few. Additionally, miRNA have been reported to play a role in the expression regulation of proteins involved in disease pathogenesis [17], stress response [5-7] and 
adaptation to extreme environmental stresses, such as freezing, at the cellular level [18, 19].

It is believed that most miRNA share a similar biogenesis mechanism. Animal miRNA biogenesis is a process which starts in the nucleus and terminates in the cytoplasm. In the nucleus, the miRNA transcription (pri-miRNA) occurs either from regions of RNA or introns, which fold onto themselves to form hairpin-like structures. They are then cleaved by enzymes called Drosha in the nucleus which create a shorter segment of around 70 nucleotides. The structure at this point is called a pre-miRNA. The pre-miRNA forms a complex with the nucleocytoplasmic transporter factor Exportin-5 and RanGTP, which facilitates its export into the cytoplasm. Once in the cytoplasm, an enzyme named Dicer processes the pre-miRNA and cuts the loop, creating an imperfect base pair miRNA duplex of approximately 20 nucleotides in length. The miRNA duplex consists of two strands of mature miRNA. Experimental evidence suggests that the strand with the less stably basepaired $5^{\prime}$ end will be kept as the mature miRNA and is later incorporated into a miRNAinduced silencing complex (miRISC), while the other pair (known as the miRNA*) is typically released and degraded. The RISC proteins bind to targeted mRNA and either mark them for degradation or inhibit their translation. New evidence has also been found indicating mechanisms for exporting miRNA beyond the cell through exosomes [20]. The structure of a miRNA is depicted in Figure 1.

The main role of miRNA is therefore to modulate expression of messenger RNA, thereby controlling gene expression. Although the exact method of mRNA silencing remains ambiguous, evidence suggests that miRNA act as cell regulatory factors at both posttranscriptional and translational levels, through mRNA degradation and translation inhibition. As the first step, miRNA target mRNA by binding to their $3^{\prime}$ untranslated region. The regulation mechanism from that point on depends on base pairing. In posttranscriptional regulation, miRNA bind to mRNA through extensive base pairing and permit 
Ago2 mRNA cleavage, which is followed by deadenylation, and finally degradation [21]. In translational regulation, miRNA bind to mRNA through limited base pairing, and by forming semi loops in the unpaired areas, thereby prevent translation from occurring in ribosomes. Experimental evidence supports miRNA inhibition of translation at both the initiation and elongation stages [21].

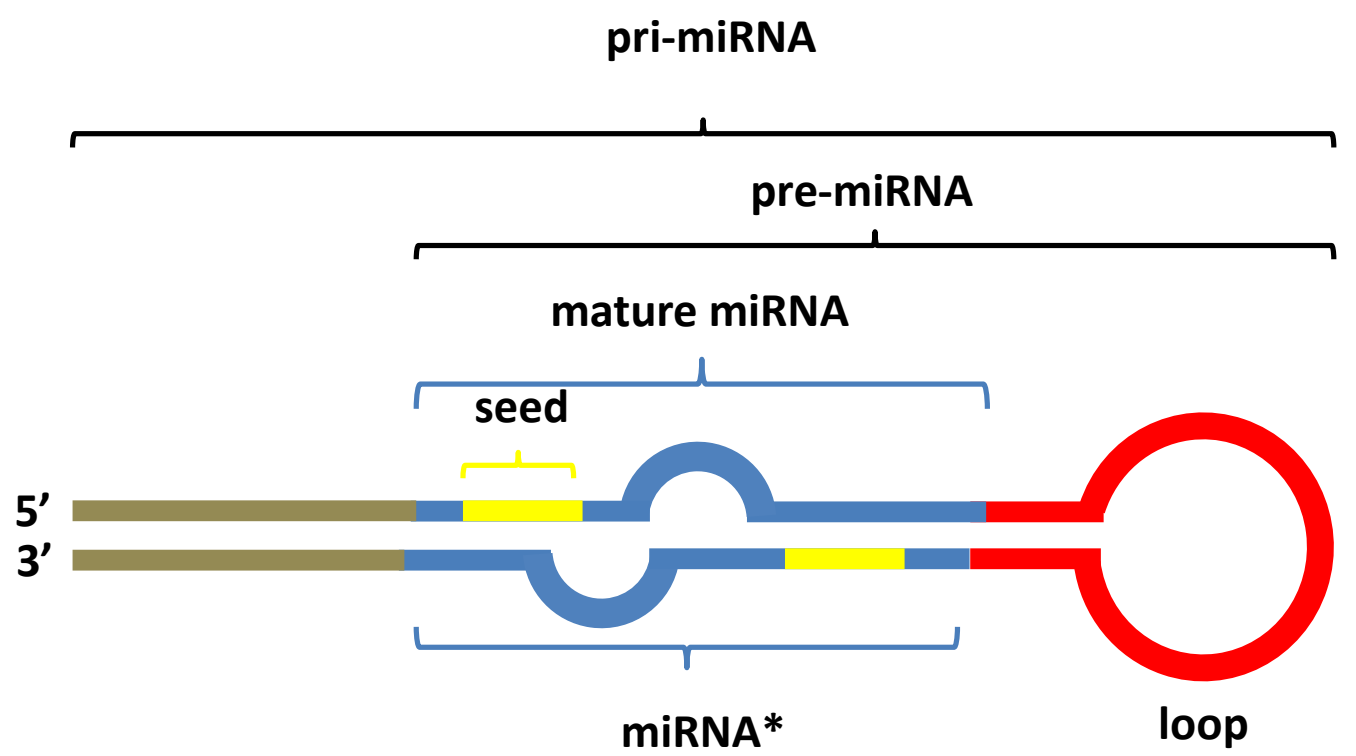

Figure 1. General structure of a miRNA (adapted from [8])

The detection and validation of miRNA is traditionally done by methods such as northern blotting [22], RT-PCR [23] and microarrays [24] to name a few. These methods are all costly with microarrays being the most expensive, while some of them such as northern blotting are also associated with contamination and low detection efficiency. More recently methods such as electrochemical methods [25], nanoparticle-derived probes [26] and isothermal amplification [27] have also been under development [28]. Although the recently developed methods have aimed to decrease the costs associated with miRNA detection, the process is still very costly and suffers low detection efficiency. 
As previously mentioned, through this silencing mechanism, miRNA target up to $90 \%$ of mammalian mRNAs, which in turn affects cell cycle control, biological development, differentiation, stress response and many more biological activities. Considering the important biological role of miRNA, identifying and accurately predicting their sequences is very valuable. Using prediction tools and conducting miRNA prediction studies can help improve our knowledge of biological pathways by discovering more novel miRNA in a costefficient way. Ultimately, identifying greater numbers of miRNA will help us better understand the biological processes within the human body and other organisms.

\subsubsection{Pattern Classification}

Recognizing patterns is a natural habit for humans, one which we instinctively repeat during everyday tasks. However, our limited memory and computational power has led us to train machines to perform such intensive pattern recognition tasks for us. Pattern classification is the technique of training machines and building models with the ability to predict the class of unlabeled data sets. Classification is based on features which are descriptive of the different classes and also have the potential to correctly discriminate between them. These features are derived from train and test samples, or exemplars, and are in the form of numerical or categorical values. A feature set consisting of $n$ features is represented using a feature vector also of length $n$. Features are utilized to devise a decision rule which is applied to all data points to classify them into one of the existing classes. Pattern classification falls into three general categories: Unsupervised learning, Semi-supervised learning, and Supervised learning. This thesis deals with the latter two approaches.

\subsubsection{Unsupervised learning:}

In unsupervised learning, the true nature of the data is always unknown, meaning that we are never certain of the correct label of the data in use. This is the more common scenario 
in real life pattern classification problems, where there is no primary knowledge or information regarding correct class labels. Therefore, the common aspects between the data points in hand become the area of focus and are used to form natural pattern groups based on the existence or absence of underlying similarities [29]. This is referred to as "clustering", meaning a number of instances are separated into clusters, where samples within a cluster should be more similar to each other than samples in different clusters. Unsupervised learning is the process of clustering or grouping samples without knowledge of any true class labels.

\subsubsection{Supervised learning:}

In supervised learning, the primary data set in hand is accompanied by the true labels of its data points, referred to as the training set [29]. The algorithm is trained to learn the characteristics of the different classes of data in the training set and create a decision rule. This decision rule is then applied to future unlabeled data sets for true class label identification. Two methods of classification exist for supervised learning; parametric and non-parametric methods. In the parametric approach, each class is modeled by making assumptions based on the underlying class distributions and estimating the model parameters using the training sample. Non-parametric methods attempt to learn the classification boundary directly from the labelled training data without making assumptions about the underlying distribution of data. A sample training pipeline for a pattern classification problem can be seen in Figure 2.

Pre-processing refers to the manipulation performed on the raw input data to extract the valuable components and simplify the data set. This step helps increase the classifier performance by removing any outliers which may distort the classifier's performance. The pre-processing techniques used for each pattern classification problem vary and are fully dependent on the problem. The feature extraction step identifies the measurable quantities 
capable of identifying patterns within the data, and aims to characterize the instances using these discovered features.

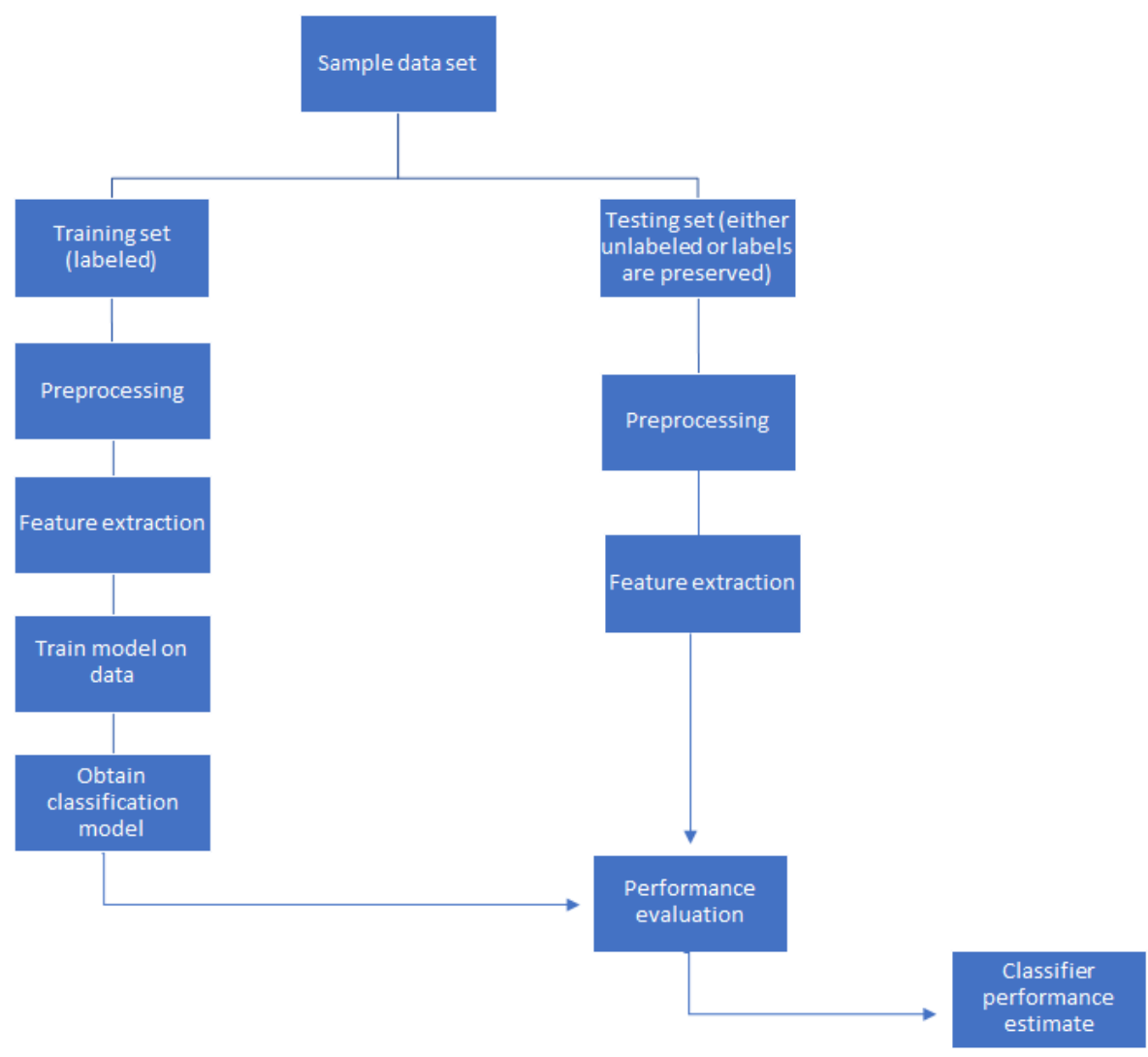

Figure 2. Sample supervised learning training pipeline

\subsubsection{Semi-supervised learning:}

Semi-supervised learning is when both labeled and unlabeled data are used for classification. Semi-supervised learning can be applied in a number of ways. For example, the unlabeled data can be used for clustering purposes and to capture the shape of the underlying data distribution and achieve a preliminary class association, while the labeled data are used to label each of the clusters based on how the few labelled training samples were distributed among the clusters. Another application of semi-supervised learning is in the case where it is possible to interactively query the true class of additional samples. Such a situation requires an all-knowing oracle that can provide the true label of any exemplar at 
a cost. In this scenario, supervised machine learning is initially carried out on a sample set and the trained algorithm is then applied to a larger set of unlabelled data. After classification, a number of the unlabelled samples (different criteria exist for this selection which will be covered in Chapter 4 of this thesis) are queried for their true class, labeled, and added to the labelled training sample set to increase the classifier's robustness. This process is referred to as active learning and is explored in this thesis.

\subsubsection{Pattern Classification for miRNA Prediction}

In this thesis, pattern classification is used to detect whether an RNA sequence represents a microRNA or not. In cases such as this, where two distinct classes exist, the two classes are commonly referred to as the negative class and positive class. Pre-microRNA hairpin therefore represent the positive class, and all other small RNA segments that are not true pre-microRNA hairpins represent the negative class or "pseudo miRNA". Since in such a problem we have access to a labeled sample set which consists of known miRNA (positives) and small RNA regions known not to be miRNA (negatives), supervised learning is commonly used for the purpose of classification. Numerous supervised learning algorithms can be used for binary classification. The goal is typically to identify the approach that most accurately distinguishes between the different class labels, making the least errors in classification. As the famous "No Free Lunch" theorem states, no single model works best on all problems [30]. Therefore, when tackling a pattern classification problem, different algorithms must be studied and tested to identify the optimal solution for that specific problem. In this regard, a brief overview of a few of the supervised learning methods which are relevant to this thesis are described below. 


\subsubsection{Decision Trees}

When faced with a pattern classification problem in real life, a natural approach is to ask a series of "yes/no" questions that lead to the identification of the object's pattern class. For example, let's say we are trying to guess what fruit is in a plastic bag. The instinctive approach we take is to start asking questions descriptive of different fruits to either identify them or rule them out, questions regarding the fruit's colour, weight, shape etc. The decision tree algorithm follows a similar concept, where it devises a sequence of questions descriptive of the instance it is trying to classify, where the next question always depends on the answer to the previous question. The answers to the questions are either "true" or "false", and these sequences of true and false questions continue until the class is detected.

The classification process in a decision tree starts with a root node at the top of the tree, with a series of branches connected to that node. All instances are checked against the root node features to determine the branch they must follow. The instance only follows the branch where the answer to the corresponding branch's question is "true". Nodes are connected by successive branches, and this process continues until a terminal node is reached that has no further branches. Such a node is called a "leaf" and provides the predicted class of the instance. A sample decision tree for the fruit example given above can be seen in Figure 3.

In addition to being simple to design and implement, decision trees have an advantage over other methods, which is interpretability. All the information in the tree can be viewed as a series of logical expressions, which provides an intuitive means to understand the model. One disadvantage can be oversensitivity to input data, such that the entire structure of the tree may be affected by a slight change in the input. Another downside of decision trees is that they often demonstrate lower accuracy compared to other classifiers, mainly due to their instability as mentioned above and difficulty in modeling class-overlap [31]. 


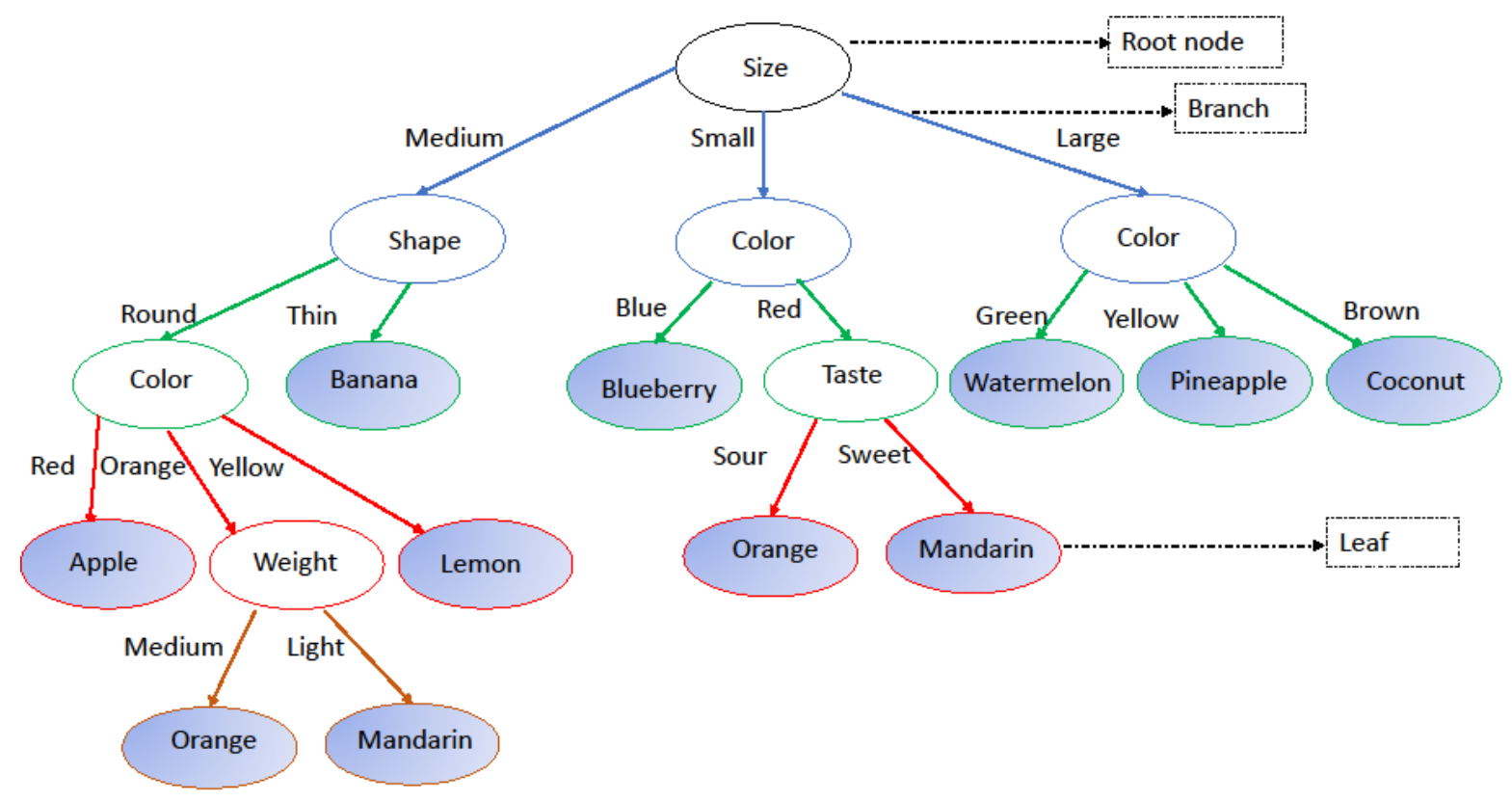

Figure 3. Sample decision tree for fruit classification (adapted from [29])

\subsubsection{Random Forest}

To overcome the instability and class-overlap issues of decision trees and still maintain their logic, the random forest classifier was proposed. Random forest classifiers are a combination of tree predictors, hence their name [32]. Since a random forest combines multiple trees for prediction, it is considered a meta-learning approach. A random forest classifier is trained by repeatedly selecting a subset of the training data and features, and using them to train individual decision trees, thereby creating numerous (in the range of hundreds) of simple decision trees. Once the individual trees are trained, the test data will be given to the trees and the final result of the classifier is determined by a vote amongst the trees. The important parameters for designing a random forest classifier are determining the number of trees, the depth of each tree, the number of data points, and the number of features used in each tree. Some criteria exist for determining the optimal parameters based on the sample set being used, but often experimental testing is applied to 
find the parameters that yield optimal performance. In this thesis, random forest has been used as the primary algorithm for miRNA classification.

\subsubsection{Support Vector Machines (SVM)}

Through training on a labeled sample set, SVM learn a decision boundary and separate the different classes using a surface that maximizes the margin between them [33]. The goal of SVM is to both maximize the margin between different classes and to generalize well on all data points, including those not in the training sample. The method follows the structural risk minimization induction principle, with the end goal of minimizing the generalization error of a model [33]. SVM classifiers have been shown to outperform other methods in many pattern classification problems.

An example of an SVM classifier for a two-dimensional training set is given in Figure 4. The solid line represents the separating hyperplane or, in other words, the classifier. The two dashed lines indicate the distance between the classifier and the closest training feature vectors. The maximum distance between the two dashed lines is referred to as the margin. Depending on the hyperplane chosen, the size of the margin will differ. This is evident in Figure 4 , as the hyperplane chosen in Figure $4 \mathrm{~b}$ results in a larger margin than that of Figure 4a. As previously mentioned, SVM aims to maximize the margin and therefore will choose the hyperplane in Figure $4 a$. As it can be observed, both hyperplanes in Figure 4 will have optimal performance when applied to this particular data set and will therefore yield the same result here; however, by choosing the larger margin the algorithm attempts to minimize the model's generalization error. This will cause the model to perform better on future data points and is the reason behind SVM's robust performance in many classification problems. SVM was used in this thesis in order to compare against Random Forest in terms of classification performance, as further discussed in chapters 4 and 5 . 
(a)

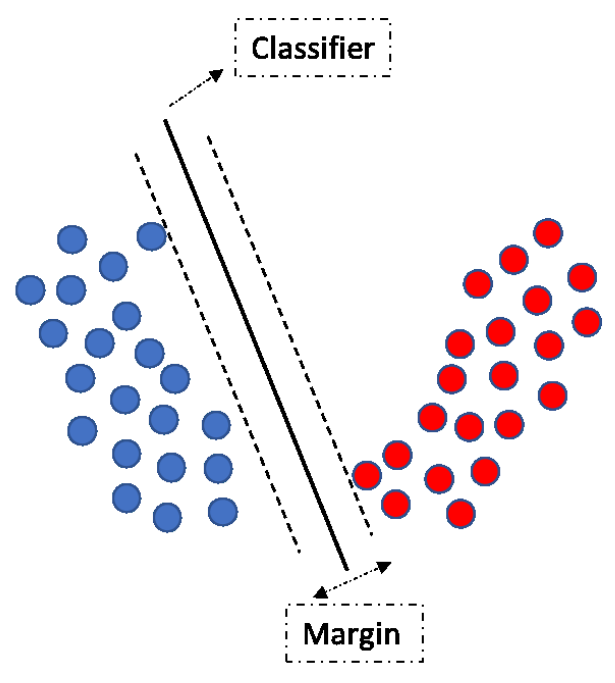

(b)

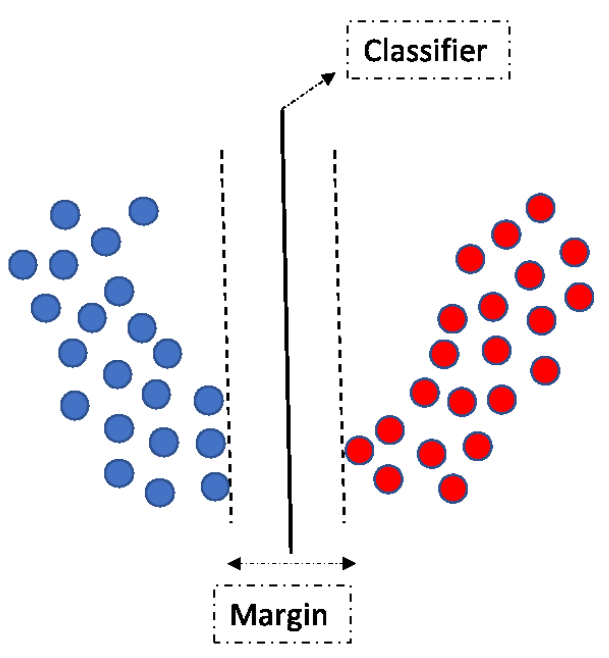

Figure 4. A Sample linear Support Vector Machine (SVM)

\subsubsection{Performance metrics}

Accurate performance estimation is a key factor, since it is a measure of a classifier's success in solving the problem, and all the steps taken in the model design are focused towards maximizing the performance with regards to these metrics. A variety of performance metrics exist and there is no single performance metric for quantifying classification performance across all problems. Each metric puts more focus on some aspects of the classifier's performance, and choosing the best metric depends on the type of problem and data in hand. These metrics are derived from the confusion matrix, which gives a complete visualization of the performance of a model, for a given decision threshold. A binary confusion matrix is demonstrated in Figure 5 , which is the basis for all performance evaluation performed in this thesis. 


\section{True class}

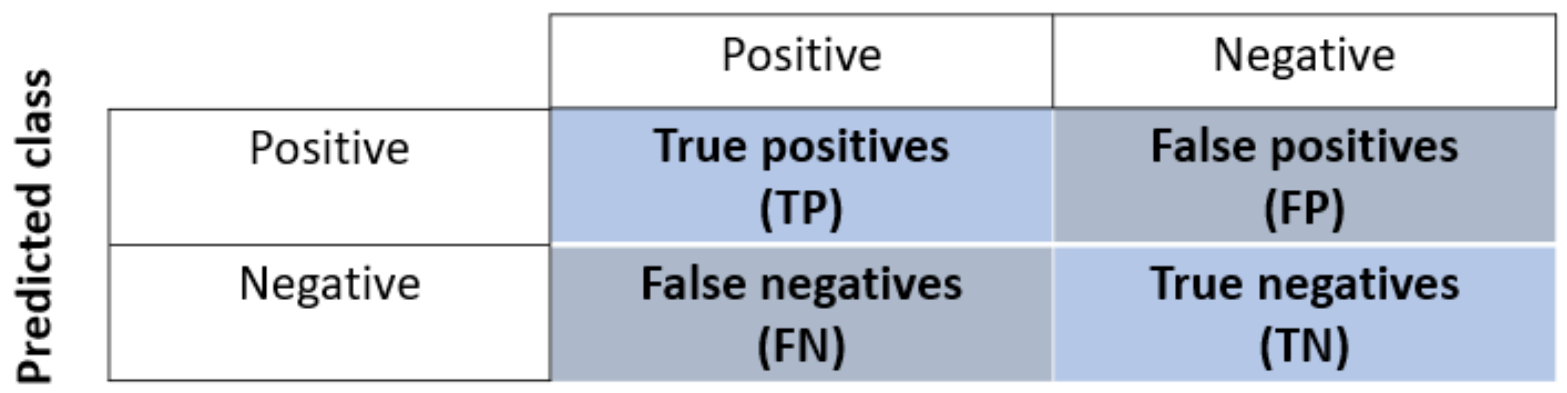

Figure 5. Confusion matrix

Many classifiers produce a continuous-valued score for each input test sample, where a higher score indicates an increased likelihood that the sample comes from the positive class. Therefore, to compute a confusion matrix, a decision threshold score must first be determined. All samples with a score above the chosen threshold will be predicted to be miRNA (positive), and all samples with a score lower than the threshold will be predicted to be a pseudo miRNA (negative). Classifier performance can be measured at various cut-off thresholds, and the final threshold depends on the performance desired by the classifier. Varying the decision threshold typically trades sensitivity (i.e. the ability to detect positive samples) for specificity (i.e. the ability to reject negative samples).

By applying a decision threshold to a predicted score, the following cases will become possible:

- True positive (TP): positive sample which was above the threshold and therefore correctly predicted (correctly predicted novel miRNA)

- $\quad$ True negative (TN): negative sample which was below the threshold and therefore correctly predicted (correctly identified not to be a miRNA)

- False positive (FP): negative sample which was above the threshold and therefore incorrectly predicted (not a miRNA but falsely predicted to be a miRNA) 
- False negative (FN): positive sample which was below the threshold and therefore incorrectly predicted (a novel miRNA but falsely predicted not to be a miRNA)

Various performance metrics can be derived from the confusion matrix and those most relevant to performance estimation in this thesis will be introduced here. The true positive rate (TPR), also referred to as sensitivity $(\mathrm{Sn})$ or recall, measures the errors on the positive set and the false positive rate (FPR) measures errors on the negative set. The true positive rate is an estimate of the probability of the classifier's performance on detecting positive samples with never before seen positive instances. The false positive rate is an estimate of the probability of the classifier incorrectly classifying a never before seen negative sample. Their equations can be defined as given below:

$$
\begin{gathered}
\text { Sensitivity }(S n)=\text { recall }=T P R=\frac{T P}{P} \\
F P R=\frac{F P}{N}=1-\text { Specificity }
\end{gathered}
$$

The total number of positives in a sample, $P$, will equal the sum of true positives and false negatives, and similarly for the negatives in a sample, $N$, will equal the sum of true negatives and false positives. Therefore, the above equations can be re-written as:

$$
\begin{gathered}
\text { Sensitivity }(S n)=\text { recall }=T P R=\frac{T P}{T P+F N} \\
F P R=\frac{F P}{T N+F P}
\end{gathered}
$$

A more commonly used metric for quantifying a classifier's performance on negative data is Specificity (Sp), which describes a classifier's ability to detect negative samples. Specificity is defined as follows: 


$$
\operatorname{Specificity}(S p)=\frac{T N}{N}=\frac{T N}{T N+F P}=1-F P R
$$

The precision of a classifier is the ratio of the true predicted positive samples to the total number of positive instances. Precision can be derived using different metrics, as shown below:

$$
\text { Precision }=\frac{\mathrm{TP}}{\mathrm{TP}+\mathrm{FP}}=\frac{1}{1+\frac{\mathrm{FP}}{\mathrm{TP}}}=\frac{1}{1+\left(\frac{1-\mathrm{Sp}}{\mathrm{Sn}} \times \frac{\mathrm{N}}{\mathrm{P}}\right)}
$$

The latter form of equation 6 is a function of the relative prevalence of the negative class, defined as $\rho=N / P$. The accuracy of a classifier is the ratio of the total number of correct predictions (both positive and negative) to the total number of instances, and can be derived as follows:

$$
\text { Accuracy }=\frac{\mathrm{TP}+\mathrm{TN}}{\mathrm{P}+\mathrm{N}}=\left(\operatorname{Sn} \times \frac{\mathrm{P}}{\mathrm{P}+\mathrm{N}}\right)+\left(S p \times \frac{\mathrm{N}}{\mathrm{P}+\mathrm{N}}\right)
$$

Prevalence-corrected precision is another metric which is used in the case where the class imbalance of the sample data in hand does not reflect the real imbalance of the data. The formula is defined as shown below, where again here $\rho$ is the relative prevalence of the negative class:

$$
\text { Prevalence }- \text { corrected Precision }=\frac{S n}{S n+\rho(1-S p)}
$$

A classifier's performance over all decision thresholds can be visualized by a Receiver Operating Characteristic (ROC) curve, which is a visualization of the trade-off between sensitivity and specificity as the decision threshold changes. An example of an ROC curve is demonstrated in Figure 6. As can be seen, a random classifier would result in a line going 
from the bottom left corner to the top right corner of the ROC curve. Increase in the classifier's performance will shift the curve towards the top left-hand corner, approaching the perfect classifier. It must be mentioned that ROC curves are not sensitive to class imbalance.

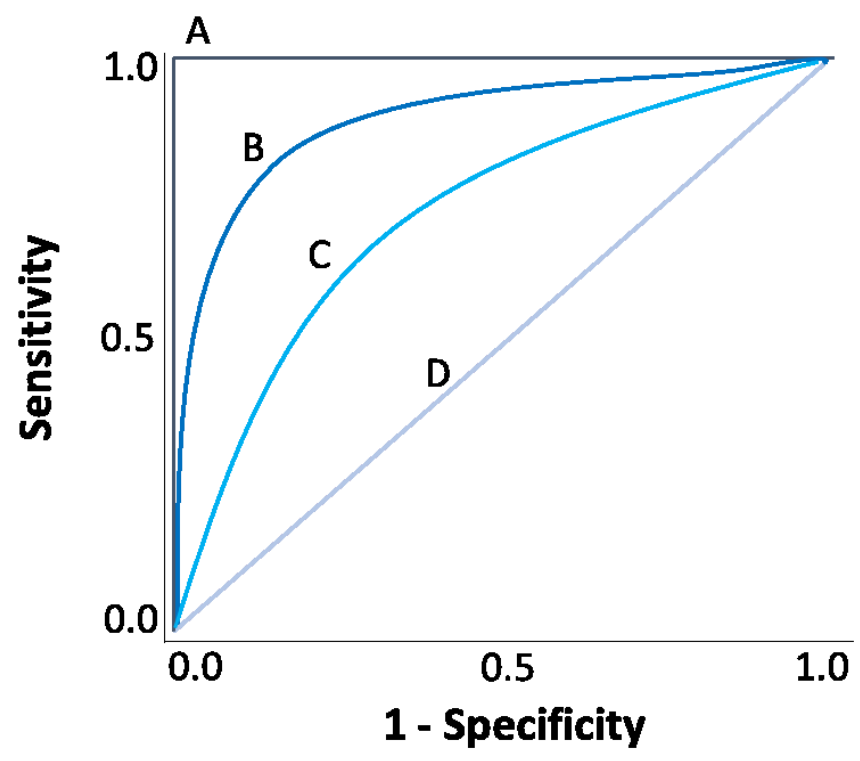

Figure 6. Sample ROC curve with different classifier performance demonstration (adapted from [34]). Curve A represents a perfect classifier, curve $B$ and $C$ represent real-world classifiers, where the performance of classifier B exceeds classifier $C$, and curve D represents a random classifier

Precision-recall (PR) curves are another performance visualization metric, where a PR curve displays the classifier's performance at different cut-off thresholds. An example of a precision-recall curve for different classifier performances can be seen in Figure 7. As demonstrated, a random classifier would result in a straight line going from the bottom left corner to the bottom right corner. Increase in the classifier's performance will shift the curve towards the top right-hand corner towards the perfect classifier. Unlike ROC curves, precision recall curves reflect the sample set's class imbalance. Each curve represents the classifier's performance at only one specific class imbalance; therefore, to obtain a better estimate of the classifier's performance on a real-world problem, the precision-recall curves should be calculated at the actual positive to negative ratio of the related problem. 


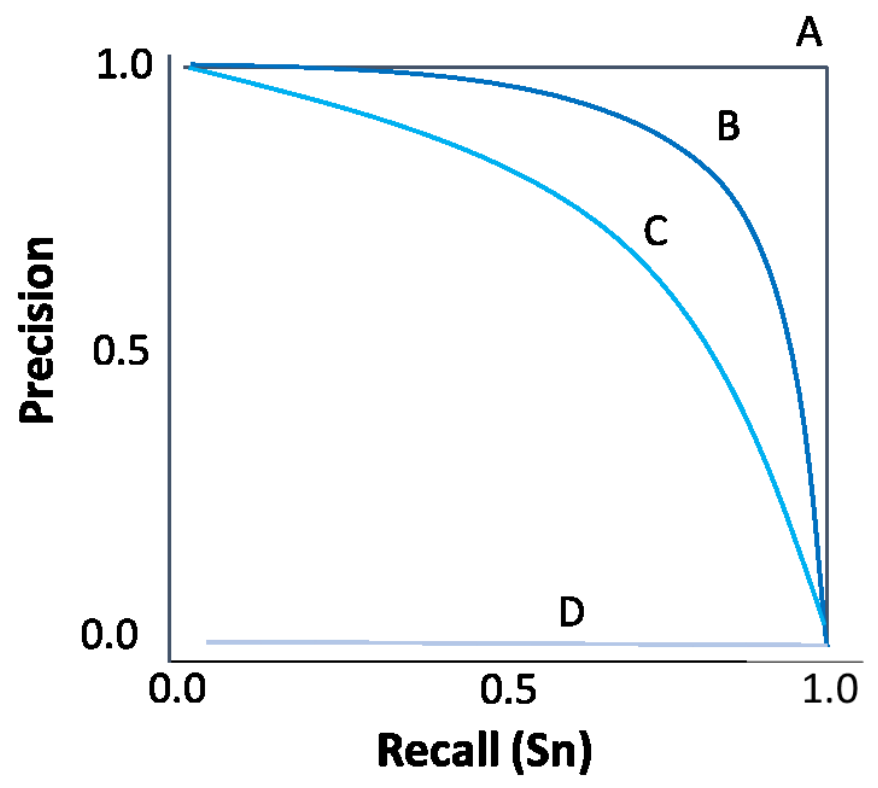

Figure 7. Sample precision recall curve with different classifier performance demonstration. Curve $A$ represents a perfect classifier, curve $B$ and $C$ represent real-world classifiers, where the performance of classifier $B$ excels classifier $C$, and curve $D$ represents a random classifier

The area under the ROC and precision-recall curves (AUC) can also be used to summarize the classifier's performance. AUC is a good estimator of a classifier's performance because it evaluates the performance over all decision thresholds. A larger AUC is representative of a better performance and hence a more effective classifier.

An ideal classifier would have both a Sn and Sp of 1, resulting in perfect accuracy. In a real world scenario, however, this is unlikely to happen, and the cut-off threshold must be set by considering a trade-off made between TPR and FPR. Determining a suitable operating threshold is a case-specific problem. A good example would be a cancer detection model, where detection of cancer would be a true positive and not detecting it would be a true negative. In such a case, a false negative would mean the subject did indeed have cancer but was not detected by the model. This will result in the treatment process being delayed, resulting in non-compensable damage to the subject's health. A false positive on the other 
hand would mean diagnosing a healthy subject with cancer. In this case, the subject will undergo more extensive testing and at some point, the initial diagnosis will be overruled. Evidently, in this scenario a false negative would have far more serious consequences compared to a false positive, therefore the cut-off threshold would be set at a more conservative score to minimize the number of false negatives, accepting that this will come at the cost of more false positives.

Such decisions are to be taken in all pattern classification problems, and such a tradeoff always exists. In the case of miRNA prediction, ultimately the predicted miRNA will be tested in a wet-lab for validation. This is a timely and costly process, especially if a high number of predictions are submitted for validation. In addition, having a low false positive rate is important because of the high ratio of negatives to positives, since most putative miRNA regions will not be actual miRNA. Therefore, a false positive is associated with time and money loss, whereas a false negative will result in the miRNA not being detected in that specific experiment. It is due to this fact that we prefer to set a more conservative cut-off score to minimize the number of false positives.

\subsubsection{Active Learning}

Obtaining a desirable performance from a classifier is generally achieved by providing it with a sufficiently-large labeled training set. In many real-world problems however, determining the true class of a training sample is an expensive process; therefore, labeled data samples come at a high price. In many cases, one has access to large amount of data (e.g. sensor measurements), of which only a small portion is actually labelled (e.g. actual seismic events among hours of seismic data). One such real-world example is miRNA prediction, where there is very limited labeled data in hand, however unlabelled (unannotated) DNA sequence data can be readily obtained through high-throughput NGS experiments. Obtaining labeled data for this purpose requires large investment in annotation, where wet-lab experiments 
are required to validate whether a small miRNA sequence is an actual miRNA (positive class) or pseudo miRNA (negative class). Unlabeled data however is abundant and easy to access, with millions of small RNA sequences extracted from different species being readily available. An ideal approach towards solving this pattern classification problem would therefore be one that makes the best use of the available unlabeled data and requires the minimum number of labeled instances to save in costs.

Active learning is a semi-supervised learning method that combines both labeled and unlabeled data to improve the classifier's performance through experience and training. Active learning iteratively determines which of the unlabelled samples would be most valuable to have labelled, for example via wet-lab experiments, such that the newly labelled sample is most likely to improve the capabilities of the classifier. The final goal is to extract the most information possible from unlabeled instances and maximize the performance of the classifier using a minimum number of labeled samples.

Different settings can be applied for active learning, of which only pool-based learning [35, 36] will be described here due to its direct relevance to this thesis. In pool-based learning, the classifier is trained using a small number of labeled instances, named the seed set [35]. The trained model is then applied to a larger unlabeled data set, called the active pool [35]. At this step, the performance of each of the active pool instances is observed and the most informative instances are selected and labeled. Once these points are labeled with their true class, they are added back to the initial labeled training set, and the classifier is re-trained using this updated training set. This cycle is repeated multiple times and, as the training set size grows with the addition of labeled data at each stage of active learning, it is expected that the classifier's performance will also improve. The model's performance evaluation is performed on a test set which is independent of the seed set and the active pool. An overview of the pool-based active learning pipeline is presented in Figure 8. 


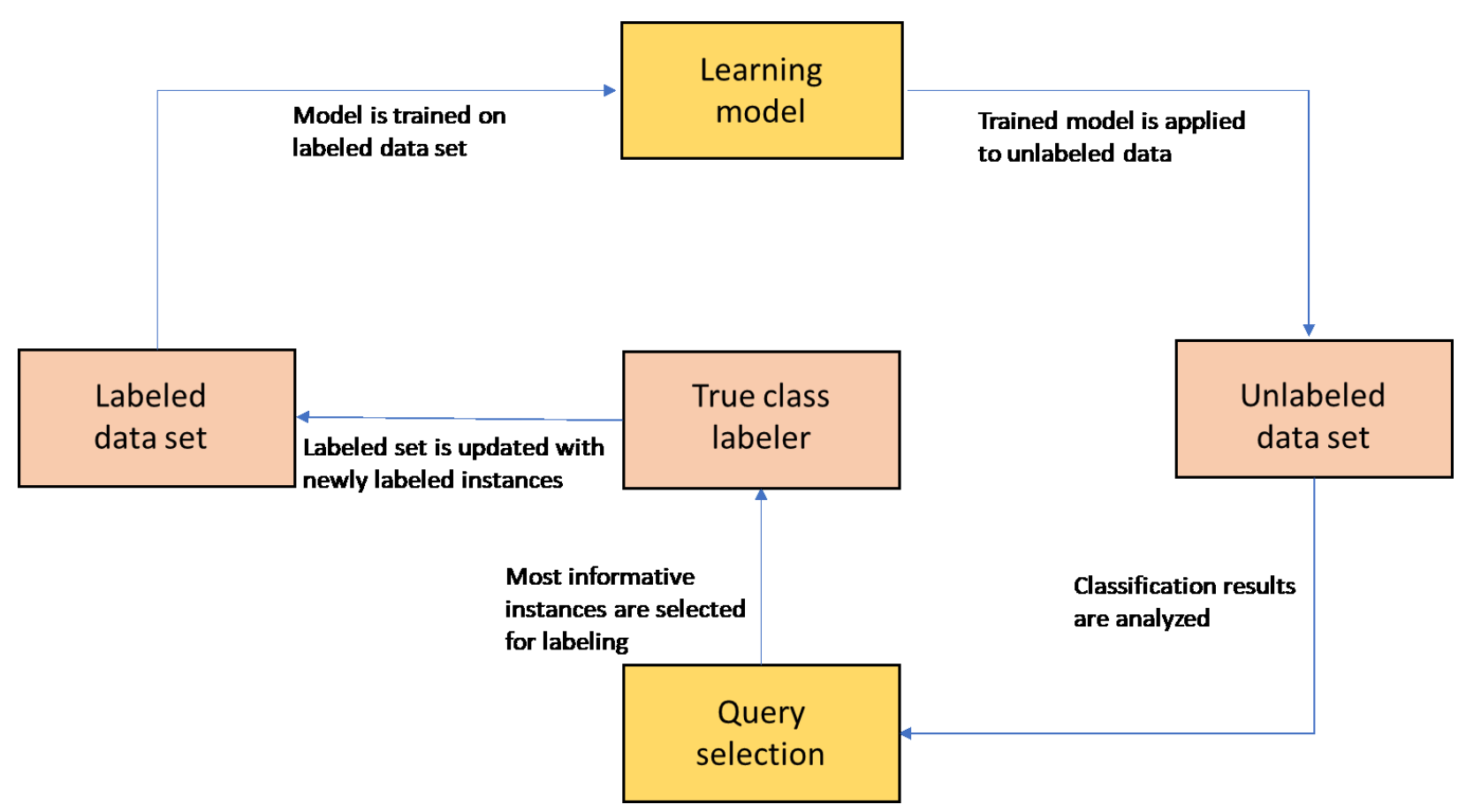

Figure 8. Pool-based active learning pipeline

\subsubsection{Query strategies in active learning}

In order to minimize the number of labeled instances required, active learning must aim to identify and label the most informative sample that will result in the largest increase in the classifier's performance. Therefore, choosing which unlabeled instance to query is an important problem in active learning. There are different approaches to determine which points are the most informative for labeling, and therefore a variety of query strategies exist. In this thesis, the Uncertainty Query Strategy and Certainty-based query strategies have been used and will therefore be briefly explained.

In the uncertainty query strategy, informative instances are those for which the classifier is most uncertain in their prediction $[37,38]$. At the query stage of each iteration, the most uncertain points will be chosen from the active pool for annotation. Uncertain points will be those closest to the decision boundary. Labeling and adding such instances to the training 
set is more likely to improve the performance of the classifier with regards to boundary points in the next iterations [39].

In the Certainty-based Query Strategy, the most confidently predicted points will be chosen from the active pool for annotation. These points will be the ones which are the most likely to have been correctly predicted to be in their respective classes [40]. In binary problems, these selected instances will have a high probability of belonging to their predicted class. Labeling and adding such instances to the training set is expected to further reinforce the classifier in more confidently predicting the true class of many of the instances, but may not increase the classifier's performance over instances falling close to the decision boundary region.

\subsubsection{Stopping criterion in active learning}

The most obvious stopping criterion for active learning is to establish a budget for labelling points before beginning, however, more advanced criteria have been proposed. With the goal of active learning being requiring the minimum number of labeled samples to maximize performance, it would be ideal to stop the labeling process at the earliest possible stage. However, it must also be noted that we want to stop the labeling process only when adding new labels will not significantly enhance the classifier's performance. Different ideas have been proposed in this regard. Zhu et al. [41] and Lewis and Gale [38] have suggested stopping the learning when the expected error of a classifier has been minimized. This maximum effectiveness and minimum error rate is determined by testing on a labeled data where the labels are preserved and only used for performance evaluation. It must be noted that the sample set used for performance evaluation must be independent of the training set and active pool, referred to as the hold-out test set. 
Schohn and Cohn [42] proposed an alternative stopping criterion and disagreed with the concept of creating a hold-out test set for performance evaluation. In addition to runtime arguments, they argued that requiring an independent labeled data set is itself contradictory to active learning's purpose, which was minimizing the number of labeled instances required. Their alternative proposal was to stop active learning once the active pool contains no more unlabeled instances close to the decision boundary (within the margin of the classifier). They explained this proposal by arguing that only instances within the margin may shift the decision boundary of an SVM classifier. Therefore, all instances within the margin have been labelled, labeling additional instances from the active pool will have no affect on the classifier's decision boundary and, hence, will not increase its performance.

Vlachos [43] made the same arguments as Schohn and Cohn [42] against creating a labeled hold-out test set to estimate the performance of the classifier, and instead suggested using the confidence of a classifier as the stopping criterion. Vlachos suggested estimating the confidence of the classifier over a separate, unlabeled dataset after each stage of active learning. The argument was that since only confidence estimation is desired and not performance estimation, this data set does not need to be annotated, and only feature extraction need be applied [41]. Whenever the confidence of the classifier drops or remains constant over a few stages, this means the remaining instances in the active pool cannot further contribute to the performance of the classifier and active learning should be stopped. He argued that, in the case of uncertainty-based sampling, since the instances added to the training set in each iteration are the most uncertain ones, eventually only instances will be left in the active pool that the classifier is confident of their labels. Therefore, at such a point, adding instances will not contribute to increasing the classifier's confidence since they do not contain new information. If, in a rare case the labels for these instances contradict the predictions, they will drop the confidence level of the classifier 
overall. Therefore, once the optimal number of points have been added to the training set, from that point on the confidence of the classifier will either drop or remain the same, notifying the user when to stop.

\subsubsection{Multi-view Co-training}

Co-training[44] is another strategy for tackling pattern classification problems in which labeling is expensive and only a small set of labeled data is available. It was previously mentioned that the ideal approach for solving such problems would be one that makes optimal use of the available unlabeled data and requires a minimum number of new annotations. The concept of co-training perfectly fits this goal description, as it aims to improve the classifier's performance without using any additional labeled data. Multi-view co-training uses the unlabeled data set to increase the size of the labeled dataset, therefore labeling instances comes at no cost. The main innovation of this method is that it creates multiple views of a classification problem, then generates different classifiers for each view, creating the advantage of being able to look at a problem from different perspectives. To better explain the concept of multi-view co-training, the same example given by Mitchell and Blum [44] in their introduction of co-training will be re-stated here.

Let us assume we are faced with the problem of classifying a webpage to either be a course homepage or not. In this case, a course homepage would be a positive instance and all other webpages will be negative instances. It would be expensive to annotate webpages manually, yet there are millions of unannotated instances freely available on the web. With co-training, a small number of labeled webpages will be created as the initial labeled data set, and a large pool of unlabeled webpages will be used as the test set. Whether a webpage is a course homepage or not can be detected from different views, and different kinds of information. One view could be the words which appear on the homepage, such as 
"outline, deadlines, course, etc.". Another view can be the words appearing on the link leading to the course homepage, such as "course, syllabus, etc.". Two different classifiers will be trained, each based on one of these views. At each step, the classifiers will be trained on the labeled set and applied to an unlabeled set. Confident predictions from each classifier will be added to the labeled set of the classifier. The models will be re-trained using a random selection of the new labeled sets and this process will continue until the desired performance of the classifier is reached. The multi-view co-training pipeline for a binary class is demonstrated in Figure 9. As it can be observed, at each stage the labeled set of the classifiers are updated by adding the top predicted instances of each view. The unlabeled sets of each classifier are also updated by removing these top points.

The successful implementation of multi-view co-training on a pattern classification problem requires a few conditions to be applicable to the problem. One evident pre-requisite is that a minimum of two different views of the problem must exist in order to be able to create a multi-view approach. Furthermore, each view must be independent of the other and in itself sufficient for correct classification on its own. In addition, the maximum effectiveness of cotraining compared to other approaches is observed when the classification problem has a small labelled and a large unlabeled dataset, and annotation is expensive. Implementation of multi-view co-training would theoretically result in successful performance on a pattern classification problem if these criteria exist. This is why in this thesis it is believed that this method can be applicable to the problem of miRNA prediction. Firstly, there is very limited labeled miRNA data available and obtaining labels would require costly wet-lab experiments, whereas unlabeled data are abundant with millions of small RNA sequences available. Secondly, current miRNA prediction methods are mainly based on either sequence-based or expression-based features (as further explained in section 2.2.1). Most methods select one of these feature sets for prediction and both methodologies have proven to be rather successful. This indicates that there are indeed two independent miRNA classification views 
available, and each view itself has proven capable of prediction. This evidence supports the view that the problem of miRNA prediction matches all the criteria for the successful implementation of multi-view co-training.

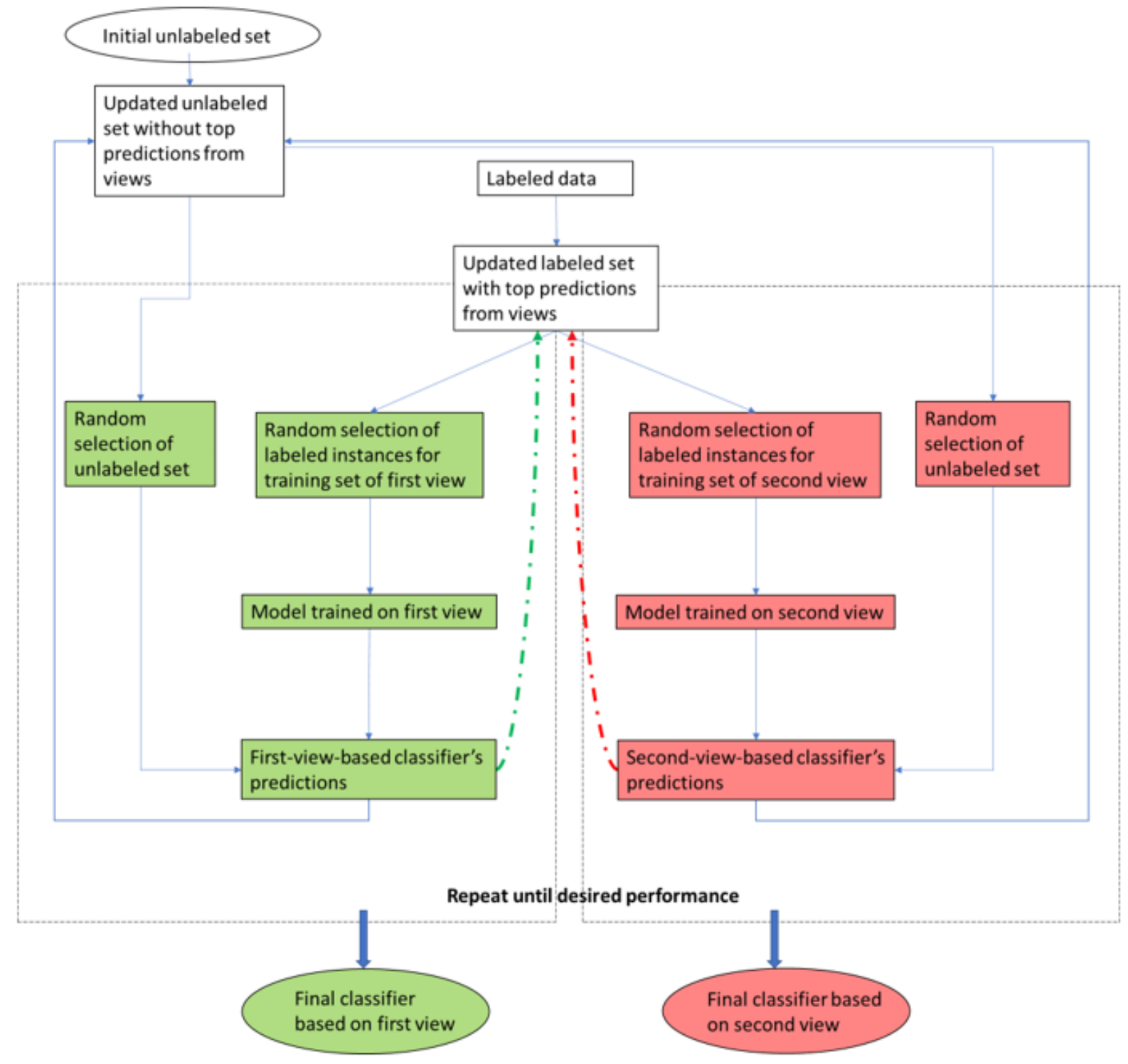

Figure 9. Binary class multi-view co-training pipeline 


\subsection{Review of the State of the Art}

In this subsection, an assessment of the state of the art will be presented for each major theme of this thesis.

\subsubsection{Prediction of miRNA}

MiRNA identification is a task which requires both experimental strategies and computational methods. The process for miRNA identification consists of first predicting the candidate miRNA from the genome sequences based on specific features (which will be further discussed), and then validating these predictions using wet-lab experimentation. Computational miRNA discovery techniques can be categorized as either homology-based, de novo miRNA prediction or NGS-based miRNA prediction. Since the work produced in this thesis is based on de novo and NGS-based prediction, they will be explained in more detail.

Homology-based techniques formed the majority of early methods and have since been largely outperformed by the other two methods. This approach relies on sequence conservation criteria and identifies miRNA homologs across species based on sequence similarities between them [45]. Amongst the first computational methods using this approach was MiRscan [46], which sequenced 3423 miRNA clones in the $C$. elegans species and detected 35 candidate miRNA genes [46]. This method also identified 107 human candidate miRNA in another experiment, while estimating the total number of human miRNA to be 250 [47]. MiRSeeker was another conservation-based method applied to the $D$. melanogaster genome, where 48 novel miRNA candidates were predicted, and it was estimated that the $D$. melanogaster genomes contained approximately 110 miRNA genes [48]. Snarloop was another similar software which was implemented on C. elegans and identified 214 candidate miRNA [49]. A number of homology-based algorithms specializing in plant homologs such as MicroHARVESTER [50], findMiRNA [51] and MIRcheck [52] have 
also been developed. This prediction strategy was mostly utilized in the first few years of computational miRNA discovery and was soon sidelined by NGS and de novo approaches. The homology approach results in the prediction of small but high-quality sets of miRNA. Therefore, although quite accurate, the total miRNA count estimates generated by this approach were later proven to be too low by the other methods.

\subsubsection{De Novo prediction}

The de novo prediction method is a more widely used approach compared to the previously mentioned. In this method, candidate pre-miRNA sequences, which are identified by forming miRNA-like hairpins, are extracted from available genomic data sets [53]. The extracted sequences are checked for different structural features and are classified based on those same features which can be extracted directly from unannotated RNA sequence data. Thus, the significant advantage of this method is that it only requires genomic sequence as input for miRNA prediction and requires no annotation. The disadvantage of this sole reliance on genomic sequence, however, is that the expression profiling of pre-miRNA will be ignored. Due to this, greater numbers of candidate miRNA must be explored, many of which may never be expressed in vivo but are still consuming extra computational space and time. Another disadvantage of relying only on the genomic sequence is the large class imbalance that is present, and this limits the performance of such classifiers [54].

\subsubsection{NGS-Based prediction}

The other main method for miRNA prediction is expression-based prediction, also called NGS-based prediction since the inputs are next-generation sequencing (NGS) experiments. This data holds information regarding the abundance of expressed RNA in a sample and can be used for miRNA identification at the transcriptome level [53]. Classification is performed 
based on features such as read depth, miRNA duplex strength and other expression-based characteristics. A diagram of a miRNA regions with corresponding read depth is shown in Figure 10. As observed, normalized read depth at each nucleotide is a strong indicator of the location of the mature miRNA. Such patterns in read depth are valuable information that can be used as features for miRNA prediction. This technique holds a great advantage over the de novo approach, since only expressed sequence regions need be examined for putative miRNA. This greatly improves the class imbalance, since the class imbalance within the transcriptome is less than the entire genome. The disadvantages compared to de novo prediction are the need for the expression profiling of miRNA which requires further experimentation, and the effect of specific experimental conditions on miRNA expression. For instance, the lifetime varies between different miRNA, meaning that some types of miRNA are more likely to be detected by NGS experiments, depending on experimental conditions.

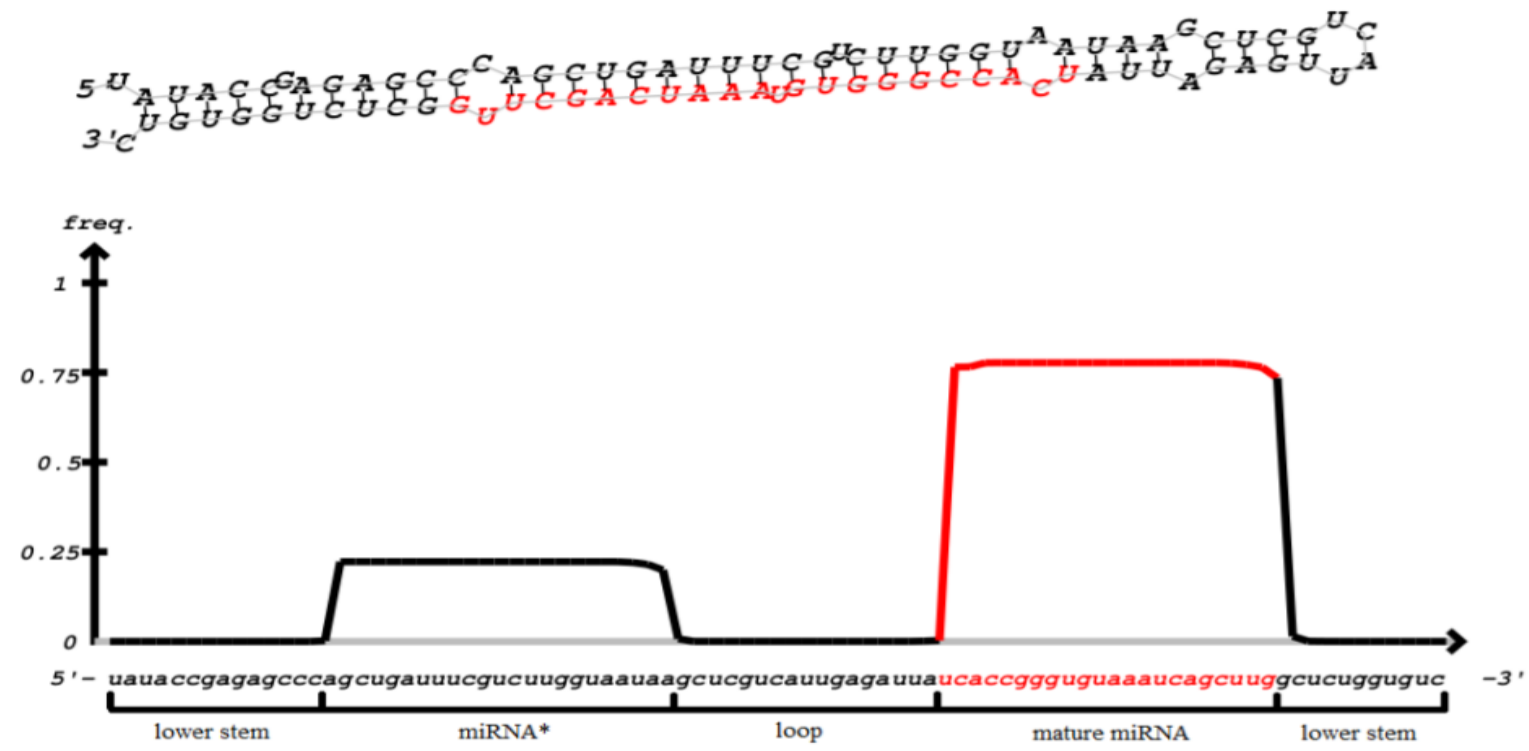

Figure 10. Information provided by NGS experiment regarding a miRNA (reproduced from [12]) 


\subsubsection{A review of de novo and NGS-based methods}

A brief review of the development trend of miRNA classification tools using de novo and NGS-based methods is presented in this section. An interested reader is referred to a recent review of the state of the art in miRNA prediction for more details [9].

In 2005, Xue et al. [55] used local structure-sequence features to classify real and pseudo microRNA precursors. They collected sets of human pre-miRNA and pseudo miRNA hairpins and extracted a total of 32 features derived directly from the genomic sequence and predicted secondary structure. These features contained information on local continuous structure and sequence information of the stem-loops, which was used to train an SVM classifier. They tested the performance of the classifier on a single human data set and achieved an accuracy of $90 \%$ when distinguishing real vs. pseudo human miRNA.

Jiang et al. developed MiPred [56] in 2007, which used a total of 34 sequence- and structure-based features for miRNA prediction, focusing on features such as the minimum free energy of the predicted RNA secondary structure and local contiguous triplet structure composition. They used these features to train a random forest classifier on a single human data set, which contained 163 real and 168 pseudo pre-miRNA. When tested on a data set containing 263 real and 265 pseudo pre-miRNA, MiPred achieved an accuracy of $91.29 \%$.

In 2009, Kadri et al. developed HHMMiR [57] for de novo prediction of miRNA. They extracted sequence- and structure-based features from pre-miRNA hairpins which provided information on region-based structure as well as sequence of the precursors. The features were extracted from a single human data set and used to train a Hidden Markov Model (HMM) classifier. When tested on a separate human data set, the method yielded a sensitivity of $84 \%$ and specificity of $88 \%$ with 10 -fold cross-validation. 
MicroPred [58] was presented by Batuwita et al. in the same year, introducing a new algorithm with a more diverse feature set for miRNA prediction. They used a subset of the features used in MiPred [56], choosing 17 sequential features, 6-folding measures, one topological descriptor and five normalized variants of those features. In addition to the 29 from MiPred [56], they considered 19 new features, focusing on minimum free energy and other secondary-structure-related features. After feature selection, a final feature set of 21 features was used to train an SVM classifier on a human data set much larger than the previously mentioned methods. They used 5-fold cross validation for performance estimation and reported a better performance than MiPred [56] on the same human data set.

A year later, Xiao et al. [59] proposed a miRNA identification method using secondary structure parameters. In this method, the structural features of pre-miRNA are extracted from their stem-loop secondary structure resulting in 24 features. These network parameters are then used to train a random forest classifier using 3000 animal samples for each of the positive and negative classes. The performance of the classifier was estimated on two independent datasets containing 1646 plant and 196 virus pre-miRNA sequences. The results showed an average AUC-ROC value of 0.944 on the test sets.

MiRDeep2 was developed in 2012 by Friedländer et al. [60] and is widely referred to as the most commonly used NGS-based analysis pipeline for miRNA prediction. This tool is an updated version of the original miRDeep [61] algorithm which was published in 2008. The algorithm consists of a pre-processing step and a scoring step. In the pre-processing step, bowtie [62] is used to map NGS data to the genome, and the regions of the reads with a length of 18 to 25 nucleotides which map to the genome are identified as candidate miRNA. Candidate pre-miRNA are identified by scanning the two genome strands of each genome at the location of the mature miRNA. This is done by once scanning 70 nucleotides from the $5^{\prime}$ 
end and 10 nucleotides from the $3^{\prime}$ end, and doing so once again but this time reversing the scan numbers (10 from the $5^{\prime}$ end and 70 nucleotides from the $3^{\prime}$ end). At the scoring step, each candidate miRNA is given a score which will be used to predict its class. A rules-based algorithm is used for the scoring process, based on features related to mature region read count, loop regions, miRNA* reads matching dicer processing and ratio of pre-miRNA region reads consistent with dicer processing. Seven species were used for training and testing the model, which included human, mouse, sea squirt, fruit fly, nematode, planarians and sea anemone. This algorithm has resulted in the identification of hundreds of novel miRNA.

MiRanalyzer, developed by Hackenberg et al. [63] in 2009 is another widely used NGSbased miRNA identification web server tool. MiRanalyzer first detects known microRNA, maps them against libraries of transcribed sequences using bowtie [62] and uses the obtained information to predict new miRNA. The features used in this method are based on nucleotide sequence, structure and energy, similar to those used by [55]. Three species (human, C. elegans and rat) which made up three different data sets were used to train random forest classifiers comprised of 100 trees. 10-fold cross-validation was used to estimate the classifier's performance and an AUC value of 0.979 was observed at a TPR of 0.79 and FPR of 0.007 when the threshold was set at 0.9 . The tool is applicable to the following seven species; human, mouse, rat, fruit-fly, round-worm, zebrafish and dog.

In 2013, Gudyś et al. presented a method called HuntMi [64] for pre-miRNA identification. They used 21 microPred [58] features, and added seven additional sequence- and structure-related attributes related to frequencies of secondary structure triplets, amino acid string length and the size of internal loops found in the secondary structure. Their training data set consisted of the same training set used in microPred [58], plus additional real and pseudo pre-miRNA regions from human, arabidopsis and numerous other animal, plant, and virus species. The aforementioned features and training set were used to train a random 
forest classifier which was applied to all the species used for training. 10-fold crossvalidation was used to assess the performance of the classifier, and the method outperformed microPred [58] on all test sets. In 2014, Zou et al. developed miRClassify [65], a web server for miRNA classification. K-grams were extracted as features for the RNA sequences, with $\mathrm{k}$ experimentally determined to be optimal at 6 , meaning 6 consecutive nucleotides were examined for each sequence. Their data set consisted of 21,264 miRNA, all retrieved from miRBase [66] and was used to train a random forest classifier. 10-fold crossvalidation was used for performance evaluation, where they obtained an average accuracy of $90.5 \%$ over the first two layers.

In 2015 Peace et al. developed SMIRP [10], a framework for microRNA prediction in nonhuman genomes. Their main goal was to sustain the performance of a human-trained classifier on other species. This is because in such instances, sensitivity is typically conserved, whereas specificity drops significantly. Therefore, they aimed to design a species-specific miRNA prediction system. The HuntMi [64] feature set was used for training the model. The following four species representing distinct phyla were created as the holdout test species for performance testing; $A$. carolinensis, D. melanogaster, $A$. thaliana and R. Iymphocryptovirus. The species-specific training sets consisted of positive data which were phylogenetically similar to each corresponding hold-out species, and the negative data were selected from closely related species to the corresponding hold-out test species. 10fold cross-validation was applied to three distinct prediction systems for performance evaluation. Support vector machines and Random Forest were trained based on three different feature sets, using human-specific and taxon-wide training data. The results demonstrated that using this approach, apart from precision and specificity, sensitivity was also sustained when estimating the performance of human-trained classifier on other species. 
In 2017, Paicu et al. developed miRCat2 [67] to predict plant and animal microRNA using NGS datasets. They proposed an entropy-based approach for miRNA loci identification to better differentiate between miRNA candidates and background sequences. The extracted features related to hairpin structure, hairpin length, maximum AMFE and precursor loop qualities. The performance of this algorithm was tested against multiple methods on different species (including mouse and human) using the same input datasets used in each method. In their results, they demonstrate that miRCat2 is able to predict more high confidence miRNA compared to other methods, most notably compared to miRDeep2 [60]. For example, the algorithm was able to identify 159 high confidence miRNA versus 149 by miRDeep2 on the human data set. It must be noted however that they reported a specificity of $78.6 \%$ and sensitivity of $30.6 \%$, compared to $94 \%$ and $26.5 \%$ for miRDeep2, respectively.

Amongst all the named tools above, none have considered using both sequence and expression-based features for miRNA prediction. The only method to do so is miPIE [12, 68], which takes advantage of the full range of evidence available for classification and prediction by integrating both sets of features. This tool uses an integrated feature set containing 223 sequence and expression-based features, and applies this feature set to six distinct species. A random forest classifier is used for all experiments, and the results are compared against miRanalyzer [63] and miRDeep2 [60]. The results demonstrate that miPIE outperforms both methods, therefore suggesting that using an integrated feature set improves classification performance.

\subsubsection{Previous applications of co-training to machine learning}

Co-training has been used in many different pattern classification problems over the past few years, especially those in which labeled data is either rare or comes at a very high 
expense. The application of co-training has mostly been focused on areas such as naturallanguage processing and signal processing, where data have multiple views, such as text data and web data [69]. Fewer contributions have been made using this approach in the field of bioinformatics compared to the aforementioned areas. One would expect greater contributions to be made in the field of bioinformatics using this method in the future, since lack of labeled data is a concern in most problems in this field. A number of the previous applications of co-training in the field of bioinformatics will be discussed in this section.

Sokolov and Ben-Hur [70] presented their findings on using a variation of multi-view cotraining for the prediction of protein function in 2011. They chose co-training as an approach to this problem because experimentally determining the function of proteins requires significant effort and expense. In addition, a variety of biological data can be used to gain information about a protein's function, such as protein sequence, protein-protein interactions, sequence similarity and gene expression. Therefore, the two main requirements for co-training are present, which are multiple independent views of a problem and a large unlabeled data set with a tendency to keep the number of required labels limited. In this model, cross-species features which characterize a protein's sequence are chosen as one view, and species-specific features which contain information about interactions and gene expression data are chosen as the second view. S. cerevisiae (yeast) and M. musculus (mouse) were chosen as the two test species. For $S$. cerevisiae, $D$. melanogaster and S. pombe were chosen as training species, while human data were used as the test species for mouse. Two SVM classifiers were trained using one view for each classifier, and at each iteration the most confidently predicted instance was added to the training pool of the other classifier. Confidence estimation was performed using 5 -fold cross validation. They compared the results of their co-training model with each of the single views and observed a higher AUC in the co-training model compared to all the other individual views. 
Note that the multi-view co-training approach of Sokolov and Ben-Hur described above differs from the original co-training algorithm proposed by Mitchell and Blum [44] in that it directly adds high confidence instances from one view to the training set of the other view, rather than having one training pool for both views. We have elected to use this newer approach in the present thesis, as described in Chapter 5.

In another application of co-training, Kim and Shin [71] targeted the prediction of breast cancer survivability using patient data. Cancer studies are greatly influenced by the lack of labeled samples, due to a variety of factors. Firstly, it takes an estimated 5 years to be able to label a patient as either a survived patient or otherwise. Second, a large debate always surrounds doctor-patient confidentiality agreements, making it difficult to gain access to samples for research purposes. If the high cost of oncology therapies is also considered, it would be reasonable to opt for a method such as co-training to deal with this problem with the least number of labeled samples required. The two classes were either survived or deceased. From a total of more than 150,000 samples, 40,000 were selected for training the classifier and 10,000 were drawn for performance evaluation. The samples were chosen randomly and without replacement. Sixteen features were obtained from the data, including tumor size, age at diagnosis, marital status, race, etc. A selection of features were chosen to train each independent classifier. After the method was implemented, its performance was evaluated and compared to three other classification techniques: ANN, SVM and ordinary single-view semi-supervised learning. Co-training demonstrated a mean accuracy and AUC of $76 \%$ and 0.81 respectively, outperforming the other three methods.

In 2015, Xu et al. [72] applied another form of multi-view co-training to help detect mislocalized proteins in human cancers using bio-images. The reason they decided to use cotraining for this problem was that large-scale biomarker screening typically lacks location 
annotations for cancer tissue bioimages, and therefore annotation is a difficult task. Therefore, they tried to make use of semi-supervised learning by creating two or three separate classifiers and using them to add labeled samples to the training set. The dataset used for training consisted of 21,920 cancer images. They also created a set of 147 images relating to 10 biomarker proteins in normal and cancerous tissues. The second dataset was used for performance validation at each stage and to help with identifying the stopping criterion. All datasets were from 11 different human tissues: breast, colon, liver, lung, lymph node, ovary, pancreas, prostate, kidney, thyroid gland, and urinary/bladder. The classifiers were trained on either high reliability, medium reliability, or low reliability level images, and at each step high confidence predictions were added to the training set. The 3classifier method included all three whereas the two classifier method used only high and medium level images. After the addition of pseudo-labeled samples to the training set at each stage, the performance was estimated on the test set they had created. The reason behind this was to stop the learning process at the stage at which the addition of newly labeled samples no longer increased performance. The results showed that, in most cases, classification performance was improved by adding medium and even low quality pseudolabeled images. This suggests that pseudo-labeling based on multiple classifiers can be effective.

In another experiment in 2013, Ibrahim et al. [73] examined applying co-training to miRNA and gene expression data for cancer sample classification. MicroRNA and gene expression data are commonly used for cancer classification and are considered to be strongly relevant to the problem. However, microarray experiments are limited, costly, and time consuming, leading to a small number of samples being available. In addition to this sample limitation, distinctive features exist for discriminating cancer subtypes, meaning the problem can be looked at from different views and, thus, co-training is a feasible method to apply. In their experiment, both self-learning and active learning were applied to the data, in addition to 
simple classifiers to detect which method performs better. In self-learning, a single classifier based on one view is trained using labeled data, and applied to the dataset for classification. The training consists of multiple stages, where at each stage a few of the most confidently predicted samples are added to the labeled training set of the initial model to enhance its accuracy. In co-training, the two different classifiers are created based on either miRNA or gene expression profiles. Three different cancer types were used in this experiment: breast cancer, hepatocellular carcinoma and lung cancer. Both random forest and SVM were applied to each method to check which one produces a better result, and they were also individually used to train models. The results demonstrated that co-training outperforms the other methods both using random forest and SVM. This suggests the advantage of using multiple views for solving a pattern classification problem within bioinformatics. Also, on average, the random forest classifier performed better than SVM over all methods.

In 2011, Du et al. [69] developed a co-training approach for classifying gene expression data called "Gene Co-AdaBoost". By nature, co-training is not a suitable method for classifying gene expression data and is expected to show limited performance improvements over self-training. This is because gene expression data by itself lacks the existence of different independent views which can be used for pattern detection. In addition, most data sets include very noisy samples which may result in the addition of misclassified samples to the training set during co-training and will disrupt the performance of the classifier. To overcome these limitations and make co-training possible, Gene CoAdaBoost split the existing gene features into independent views. To split the features into independent views, the genes were ranked with signal to noise ratio, the most informative of the genes were selected and then they were separated into multiple views. Additionally, to overcome the noise issue, a cascade structure classifier [74] was used to decide whether to add a predicted sample to the training set or not to avoid adding misclassified points to the training set. Learning was applied until there were no more unlabeled samples left. The 
model was trained on leukemia, colon, lung and ovarian expression datasets. They compared their results against three other semi-supervised learning algorithms: Tri-training [75], a Semi-Gaussian Fields Approach [76] and Self-training. A combination of 3-NN, Naive Bayes and decision trees were used for classification. The results verified that Gene CoAdaBoost performed better compared to the other three methods. Analyzing the method's performance at different stages of learning also indicated that the addition of the cascade structure classifier was helpful in sustaining performance and preventing the addition of mislabeled samples to the training set.

Roqueiro et al. [77] applied co-training to in silico phenotyping in order to improve phenotype prediction from genotype. Disease phenotype prediction requires data from both genotyped and phenotyped patients. Genotyping has become very common and therefore strong datasets have been created, however this is the case for phenotyping. This imposes a serious limitation for disease phenotype prediction in terms of data availability. To overcome this issue, Roqueiro et al. proposed using a second view for phenotype prediction, which is the patient's clinical health record. Their idea was that, by integrating two views using co-training, missing disease phenotype data can be compensated using the patient's genotype. The dataset used in this experiment consisted of data from patients with two types of migraine: migraine patients with and without aura. The dataset included clinical covariates and genotype data for all patients. Bagging predictors [78] were used as classifiers, where the goal of the final classifier was to correctly predict which type of migraine each patient should be diagnosed with. The training data included a set of clinical covariates and genotype data, whereas the evaluation dataset contained the patients genotype and disease phenotype. The results demonstrated that implementing co-training resulted in higher phenotype prediction accuracy in cases where patients were missing disease phenotypes. 
Despite the mentioned applications of multi-view co-training in the field of bioinformatics, this approach has never been used for miRNA prediction. In the field of miRNA prediction specifically, very few of the existing prediction methods have taken advantage of the full range of evidence available for classification and prediction, and none of the methods have focused on the multiple views available for approaching this classification problem. The problem of limitations in prediction validation and number of known miRNA have also always limited the performance of existing methods, especially in less-sequenced species. The existence of two independent views of the problem and the high expense of annotation make co-training a very suitable approach for miRNA prediction. Co-training holds the ability to use each distinct view of this problem to strengthen the other without the need for any extra annotation in the process, therefore taking full advantage of all the existing evidence to reduce the need for labeling. With the growing availability of unlabelled miRNA data due to NGS experiments, co-training is a valuable approach for taking full advantage of this readily available data, especially for species with only a handful of known miRNA. Chapter 5 of this thesis is dedicated towards implementing this approach for miRNA classification for the first time.

\subsubsection{Active learning in bioinformatics}

Active learning has been more widely used in the field of bioinformatics compared to cotraining. Among the earliest applications of active learning to bioinformatics, in 2002 Forman suggested applying active learning to studies in the field of chemistry and specifically to reduce biochemistry lab costs in drug discovery experiments [79]. The goal of these experiments are to identify the organic compounds that bind to a target site (labelled as the positive class). Therefore, biochemists will be in search of all the positives in a large data set. Applying active learning would improve the current experimental method by identifying the positive instances amongst a large number of samples and reducing the laboratory testing required, rather than using the entire data set for training and testing. An 
experiment was performed on a set of organic compounds to predict which samples bind to thrombin, which is a protease related to blood clotting. The data set consisted of 634 compounds, of which 150 were known to be active in binding and a total of 8000 features were used (the experiment was also separately conducted using 2000 features). Three classifiers were used in three distinct active learning experiments to determine the best classifier, which included Naïve Bayes, Nearest Neighbor and SVM. The classifiers were also compared with a random active learning algorithm. The performance of these classifiers were compared based on plots demonstrating the number of positive binding compounds found over time, in addition to ROC curves. 600 iterations were performed, and the results demonstrated that all active learning algorithms performed much better than the random algorithm. Within the classifiers, SVM had the best performance and Naïve Bayes was second best.

In 2004 Liu et al. [80] proposed using an active learning approach for gene expression profiling of cancer to minimize the labor intensive and expensive task of labeling instances. The data set used for learning consisted of gene expression profiles of colon, lung and prostate cancer samples. Both active learning and passive learning were applied to the data set for performance comparison. In passive learning, the samples chosen for annotation and addition to the training set were selected randomly, whereas an uncertainty query strategy was used for active learning. Both methods were trained using SVM, and their classification performance was compared. The results showed that, given the same number of labeled samples, active learning produced an AUC of 0.81 compared to only 0.50 for passive learning. Active learning greatly decreased the number of annotations required for a specific performance score over all three data sets, with the best results being achieved on the lung data set with an $82 \%$ reduction in annotations required. For lung cancer classification, active learning required only 31 annotations to predict $96 \%$ of the total positives in the data, whereas passive learning required 174 annotations to yield the same performance. 
Overall, the results demonstrated how active learning can reduce annotation costs while achieving high accuracy at the same time.

In 2009 Doyle et al. [81] developed an active learning framework to accurately detect cancerous regions on prostate histopathology samples. The complexity of histopathology images and the need for expert medical knowledge for diagnosis make the annotation process relatively costly. In addition, within this type of data, typically one of the classes, which is understandably the cancer class images in this case, are underrepresented. To overcome these problems, class-balanced active learning (CBAL) was applied to this problem, to make optimal use of manually annotated histopathology images and to address the minority class problem. Analyzing 100 images resulted in a dataset of over 12,000 prostate images, and 14 highly discriminating features were extracted from these images to be used for training decision trees and SVM. By applying CBAL, at each query stage equal numbers of instances from the two classes (cancer or normal) were added to the training set from each class. Depending on the experiment, the learning continued until either the unlabeled set became empty or the maximum number of iterations set as the stopping criteria were reached. The results demonstrated increase in accuracy and AUC compared to passive learning. The method achieved a similar accuracy to the other methods by using two orders of magnitude fewer samples (50 compared to 1200). Therefore, the method increases accuracy as well as reducing annotation cost.

Mohamed et al. [39] used active learning for human protein-protein interaction prediction in 2010. Protein-protein interactions are believed to be useful in understanding disease mechanisms leading to drug discovery. The problem is that out of the expected hundreds of thousands of interactions that take place, only around 40,000 are known and validating predicted interactions requires wet-lab experiments. This means a strictly limited labeled dataset is in hand, making active learning an understandable choice for use as a prediction 
technique. The goal of a classifier in this problem would be to correctly label a protein pair as either interacting or non-interacting. The data set consisted of 14,600 pairs of interacting protein pairs referred to as the positive class and 400,000 non-interacting pairs as the negative class. Different active learning algorithms were created using random forest classifiers, using uncertainty-based query sampling and density seed active learning strategies. These methods were compared to the case of randomly adding samples to the training set. For all algorithms, 250 samples were annotated and added to the training set in each iteration. Twelve iterations were performed in total, resulting in a total of 3000 labeled samples being added to the training set. The results demonstrated that all active learning algorithms performed significantly better than the random method, by needing only 500 queries to achieve a higher harmonic mean of precision and recall score than 3,000 randomly chosen samples. This indicates a $15 \%$ increase in performance when using active learning compared to random sample selection. The results of this experiment suggest that active learning algorithms may result in more accurate classifiers while requiring significantly less labelled data.

In another study, active learning was used for the characterization of endothelial cells in human tumors [82]. Visual identification of endothelial cells is difficult due to their low numbers and dispersal throughout tumors, and biomarker staining is necessary for identification. Biomarkers however make the visual inputs more complex, and their analysis requires expertise, time and expert supervision. These limitations demand a method which can minimize the number of labeled samples required and therefore active learning is applied to the problem in this study. To build the model, 8,360 human cells were segmented by a nuclear segmentation algorithm and were then manually classified by a pathologist. A logistic regression classifier was used and the number of iterations for active learning was set to 50. The results of this method were compared to a logistic regression classifier trained on the same data but without active learning. The results demonstrated that the 
active learning algorithm outperformed the simple classifier in both specificity and sensitivity.

Pertusi et al. [83] used active learning to create a larger training set to use for the prediction of novel substrates for enzymes. Enzymatic substrate provides important information for understanding metabolism and bio catalysis, which explains the importance of characterization of enzyme promiscuity. This is currently done with a restricted number of compounds which are not guaranteed to fully represent the enzyme's versatility. Testing large numbers of compounds is costly and difficult, and therefore this study suggests using active learning on existing data to identify informative substrates for testing. The purpose of this study is to expand the labeled data set using active learning rather than performing classification. In the study, active learning was applied to 4 data sets, where SVM was used as the classifier and one iteration was performed per enzyme. The performance of this model was compared to a random learning classifier, where points were added randomly at each iteration. It was observed that active learning was successful in adding highly informative data to the training set, and greatly outperformed the random algorithm in doing so. This enlarged training set was then used to train SVM classifiers. The overall method resulted in reaching a maximum accuracy of $80 \%$ using $33 \%$ fewer compounds than existing methods.

Xiong et al. [84] also used active learning on proteins, this time for protein function prediction in protein-protein interaction networks. As previously mentioned, such problems will not have optimal performance when labeled proteins are limited, therefore strategies which minimize the number of required labeled data and increase the training data, such as active learning are preferred. Similar to the previously mentioned study, active learning was used to increase the size of the training set for better classification. After the training set was enlarged, a collective classification algorithm was applied to this set to predict protein 
function. The results demonstrated that this approach performed much better than the baseline approach which did not apply active learning. Iyuke et al. [85] also applied active learning to a protein related problem, where they identified hydroxylation sites on proteins using this method. They tested different active learning strategies, such as certainty and uncertainty-based query strategies, using SVM classifiers to predict asparagine/aspartate hydroxylation sites on proteins. Their results showed that active learning using an uncertainty-based selection criterion, and a density-based approach to forming the initial labelled seed set, was the best active learning strategy and highly effective for identifying hydroxylation sites on proteins.

In another protein-related study, Reker et al. [86] used active learning for computational chemo genomics. Computational chemo genomics has wide applications in drug discovery, since it models the compound-protein interaction space. Existing computational chemo genomics approaches model large datasets to incorporate more features, whereas by using active learning fewer yet more informative examples can be used for modeling. Random forest classifiers were trained on ligand-target bioactivity datasets. 10,000 iterations were performed in the active learning process, with the goal of terminating the learning when approximately 10 to $20 \%$ of the most informative compound-protein pairs were labeled and added to the training set. From the results it was observed that by using active learning, predictive models can be developed which have the same performance of a model trained on a large bioactivity dataset but using only approximately $25 \%$ the data.

As it can be observed from the aforementioned examples, active learning has been implemented in a variety of bioinformatics related studies. Active learning benefits from a low demand of initially labeled examples and provides an effective strategy for spending an annotation budget on the instances which maximize classification performance. These factors become especially important in areas of research where annotation comes at a large 
cost, or where labeled data is scarce in general. These facts hold true in miRNA prediction where, for less-sequenced species, there are very few known miRNA to use for classification. For species that have been well sequenced such as human or mouse, there is a high cost associated with validating new predictions through wet-lab experiments. Therefore, active learning is proposed as a suitable machine learning approach for miRNA classification. In Chapter 4 of this thesis, we use active learning for miRNA classification and report the results. 


\section{Data collection and experiment setup}

\subsection{Introduction}

In order to apply any machine learning algorithm to a problem, a dataset containing labelled data from each class of the problem is required. The accuracy of its labeled samples is one of the main factors contributing to any resulting classifier's performance. A single misclassified instance in the training dataset can cause the classifier to learn to misjudge all similar instances, therefore significantly decreasing performance. It is important to gather a comprehensive data set which includes a wide variety of instances so that the classifier can generalize well on all future instances. In addition to the data set, the feature set used for training an algorithm also plays a vital role in a classifier's performance. It is critical to use a feature set that is descriptive of the instances, while also permitting discrimination between samples from each class; failing to do so would result in instances being classified based on criteria which are insignificant towards identifying the correct pattern.

In this chapter, the data set and feature set selection and preparation are described. Data sets of RNA sequences from distinct species were used to extract the positive and negative instances to use in classification. Positive instances were known pre-miRNA sequences and negative samples were pseudo miRNA sequences. These data sets were used to extract suitable features. In addition, the software aspects of our experiments is briefly explained.

\subsection{Data set selection}

To create the data sets, a large number of RNA sequences were required. Not all RNA sequences were desired, however, as only sequences that resemble miRNA would be useful for training as the negative and positive data. Therefore, in order to have a more relevant preliminary data set in hand, experiments with small RNA sequences were selected. Six 
sample datasets were download from the NCBI GEO database [87], from six different species: human, mouse, fruit-fly, cow, horse and chicken. The datasets used in this thesis are presented in Table 1. These species were selected since there are sufficient miRNA and known non-coding functional RNA data available for them, allowing us to construct robust positive and negative data sets.

\begin{tabular}{c|c|c|c|} 
Data set & Accession id & Organism name & Number of reads \\
\hline hsa (human) & GSM1820470 & H. sapiens & 38210937 \\
mmu (mouse) & GSM1528810 & M. musculus & 54947527 \\
dme (fruit-fly) & GSM1123781 & D. melanogaster & 18723989 \\
bta (cow) & GSE74879 & B. taurus & 43164654 \\
gga (chicken) & GSM2095817 & G. gallus & 27937224 \\
eca (horse) & GSE100852 & E. caballus & 42178766 \\
\hline
\end{tabular}

Table 1. List of datasets used in experiments

In addition to RNA sequences, the sequences for all known miRNA were downloaded from miRbase (release 22) [8] and the full genome for each species was downloaded from the UCSC genome browser database [88]. Only high-confidence known miRNA were selected for download from miRbase. It must also be noted that all the downloaded data used in this thesis were converted to FASTA format for further manipulation. The genome data and the data presented in Table 1 were used to obtain a positive and negative data set for each of the species. This was done using some of the functions provided in the miRDeep 2 software [60]. miRDeep2's "mapper.pl" algorithm was used to map each read stack from the NGS data to the reference genome of the species. The algorithm removes redundancy by collapsing reads that have identical sequences, and shows the number of times a sequence was present in the data set in the resulting file. The algorithm also predicts RNA secondary structures for each of the mapped regions. 
When the mapping is done, either a score is obtained for each of the potential precursors or they are discarded. The precursors are discarded if less than $60 \%$ of its mature parts' nucleotides are base-paired, since true miRNA have a significant base-paired "stem" (see Figure 10). The score threshold can be adjusted by the user; the default value was used in this thesis. The final output of this algorithm is a set of candidate pre-miRNA accompanied by their pre-miRNA structure, sequence, and set of reads mapping to the sequence. All these candidate pre-miRNA were then compared to all known miRNA sequences retrieved from miRbase for that species. Candidates that matched to a known miRNA sequence were selected as true positives. All the other candidate pre-miRNA which did not map to a known miRNA were retrieved to create a candidate negative set. This candidate negative set was then aligned against the coding region data of the related species, which was downloaded from the Ensembl sequence FTP database [89]. The mapping was performed using bowtie [62] and a maximum of two mismatches were allowed. All the sequences from the candidate negative set that aligned to a coding region were selected as our final true negative data, since sequences that are known to be mRNA fragments are unlikely to also form miRNA. This procedure was performed to ensure that no actual miRNA that have gone undetected by the miRdDeep2 algorithm are in our negative dataset. By performing this alignment, only candidates which are known to be coding regions are selected, therefore they cannot be miRNA, since a miRNA is by definition a small "non-coding" RNA. In addition to these sequences, a number of functional non-coding RNA were downloaded from Rfam [90] and added to the negative data sets to make sure the algorithm would not be trained to detect only coding regions as negatives. These non-coding RNA have already been identified not to be miRNA, but rather other type of functional non-coding RNA such as Transfer RNAs (tRNA) and Small Nucleolar RNAs (snoRNA).

After obtaining the positive and negative datasets, the CD-HIT Suite [91] program was used to remove redundant and highly similar sequences. The sequence identity threshold was set 
to $90 \%$, and all other parameters were set as default. This resulted in a decrease in the number of true positive and negative samples in some species. This step is important since we do not want to have nearly identical sequences in both our training and testing data. Table 2 includes a list of the number of true positives and negatives derived for each of the species using the method explained. The final number of true positives and negatives used throughout this thesis is the numbers that are given after using CD-HIT. The preparation of each data set required between 16 to 20 hours, depending on the data set size.

\begin{tabular}{c|c|c|c|c} 
Data set & $\begin{array}{c}\text { \# of positive } \\
\text { samples }\end{array}$ & $\begin{array}{c}\text { \# of negative } \\
\text { samples }\end{array}$ & $\begin{array}{c}\text { \# of positive } \\
\text { samples after CD- }\end{array}$ & $\begin{array}{c}\text { \# of negative } \\
\text { samples after CD- }\end{array}$ \\
& & & HIT \\
\hline Hsa (human) & 553 & 950 & 509 & 842 \\
\hline mmu (mouse) & 384 & 872 & 367 & 844 \\
\hline dme (fruit-fly) & 110 & 97 & 110 & 97 \\
bta (cow) & 341 & 683 & 332 & 650 \\
\hline gga (chicken) & 193 & 104 & 193 & 104 \\
\hline eca (horse) & 364 & 228 & 364 & 224 \\
\hline
\end{tabular}

Table 2. Number of samples in positive and negative data sets

\subsection{Feature set selection}

Different feature sets were used in chapters 4 and 5 of this thesis, therefore the description of the final feature set selection used in each chapter will be discussed in that chapter itself. But generally speaking, in this thesis both expression-based and sequence-based features are used for miRNA prediction. The sequence-based features are obtained from HeteroMiRPred [92], which has developed a large feature vector drawn from previous studies and also their own features. Their feature vector includes sequence-based, secondary-structure-based, base-pair-based, triplet-sequence-structure-based and structural-robustness-related features. Using this feature vector provided us with 215 
sequenced-based features. Eight expression-based features derived by [12] were also considered for addition to the full feature set, which contain information on the mature and lower stem regions and the expression pattern of miRNA region. In Chapter 4 , these features are combined to create an integrated set of 223 features for active learning experiments. In Chapter 5, these expression- and sequence-based features provide our two independent views of the problem for co-training.

\subsection{Experiment software setup}

All algorithms developed and implemented in this thesis are implemented using the scikitlearn package [93] in Python. This package includes a large variety of data analysis tools and classification algorithms. The NumPy library, which is a suitable framework for the handling and manipulation of large data using multi-dimensional arrays, is also used. All instances and their features are stored in NumPy arrays for each experiment, whereas they would be too large to handle using normal Python arrays. During our experiments, we developed numerous SVM and Random Forest classifiers. These classifiers are built using the SKLearn SVM and SKLearn random forest libraries. Standard values are used for classifier parameters without being tuned in order to avoid over-fitting to our particular datasets. All SVM classifiers are built using the SKLearn SVM library's default parameters, with the kernel set to linear. Similarly, for all the random forest classifiers used in this thesis, the maximum number of trees is set to 500 and all other parameters are set to the default of SKLearn. In the default settings for the random forest, the maximum depth of each tree is set to "none", meaning that nodes are expanded until all leaves contain less than two samples. The number of features to use in each tree is set to the square root of the number of features. Bootstrapping is used when building the trees and the minimum number of samples for a node split is set to 2 . The deployment of our method required between 4 to 6 hours depending on the data set size using a $3.30 \mathrm{GHz} 12$ core processor. 
In all the classifications we perform on putative miRNA regions in Chapters 4 and 5 , a prediction score is given to each instance. This score is the classifier's prediction of the class probability of that instance. This score is obtained using SKLearn's "predict_proba(X)" function. For random forests, this probability represents the mean class probabilities of the decision trees forming the random forest. The probability is given as a percentage between 0 to 100 for each class, which represents the probability of that instance belonging to either class. For example, if an instance has a probability score of $90 \%$ for the positive class, it indicates that the classifier gives a $90 \%$ probability that the instance is a miRNA, and a $10 \%$ probability that it is not. Therefore, all instances having a $50 \%$ or more positive probability are viewed as predicted miRNA, and the rest which are below that score are viewed as predicted pseudo miRNA. This probability score is used as a basis for both active learning and co-training strategies implemented in Chapters 4 and 5 of this thesis.

\subsection{Conclusion}

In this chapter, we provide an overview of the data set selection applied for the six species used in our experiments. RNA deep sequence reads, known miRNA regions, and the complete genome sequence for these six species were obtained and processed through the explained methods to extract positive and negative instances required for classification. The general feature set selection methods are also briefly discussed; since we use different features for classification in our proposed active learning and co-training frameworks, further discussion of this topic is provided in the related chapters. In general, the same data sets that are developed are used to extract the most informative features descriptive of premiRNA sequences, and form our feature sets. The software implementation of our methods are also discussed. All classifications performed in this thesis are developed and implemented using Python's scikit-learn machine learning tool [93]. 


\section{Active Learning for miRNA Prediction}

\subsection{Introduction}

MiRNA are difficult sequences to experimentally validate, considering their short size of around 18 to 22 nucleotides and their degenerate sequence. Wet-lab validation experiments are expensive, difficult, and error-prone. The current state-of-the-art miRNA prediction tools generally require a large set of known miRNA sequences. For some species, where there is already a large number of identified miRNA, this may seem quite feasible. Although, even for such species, the improvement of miRNA prediction methods requires the constant increase in the number of known miRNA, and multiple experimental validations for the predictions made. The problem becomes more complex when dealing with species with less data availability, where a large cost must go into first identifying enough data for primary predictions and then experimentally validating them. These experimental validations are very costly and repeating them several times for acquiring labeled data would not be feasible. Considering the high costs associated with experimental validation of miRNA, we would at least want to perform these validation experiments on instances which will maximize performance increase. Considering these factors, a classification method which minimizes labeling costs, chooses the most informative instances for labeling and at the mean time maintains performance would be ideal.

We hereby propose the use of active learning for miRNA classification to make the most efficient use of wet-lab experimental validation of miRNA. Active learning is a semisupervised machine learning approach; therefore, it does not require as much labelled data as other supervised methods currently in use. In addition, by using active learning, we have the potential to maximize the improvement in our classifier's prediction accuracy using a minimum number of informative annotations. In this chapter, we will study the effectiveness 
of applying active learning to miRNA classification. We aim to study how utilizing this approach can limit the number of required labeled instances while increasing classification accuracy. We will also consider different active learning strategies, in order to find the optimal strategy to maximize classification performance in return for the resource we spend when labeling new data. Furthermore, this thesis also explores another novel approach to miRNA prediction, which is the combination of expression and sequence-based features for prediction. This idea was first proposed in Robert Peace's PhD thesis [12] and is further explored here and in a separate publication currently under peer review [68].

As mentioned in chapter 2, de novo and NGS-based miRNA prediction techniques are currently the leading computational methods for miRNA prediction. These techniques detect pre-miRNA sequences forming miRNA-like hairpins and classify them based on the presence or absence of sequence or expression-based features. Each of these classification methods hold some advantages over the other and still have deficiencies for high-performance miRNA prediction. De novo prediction methods hold the significant advantage of only requiring genomic sequence as input; however, they suffer from large class imbalance and do not leverage expression profiling information. NGS-based prediction methods require transcriptomic data in addition to the genomic sequence but, in turn, improve class imbalance and only require the evaluation of expressed regions as opposed to all the putative regions. Most current miRNA classification tools only use one line of evidence, being either sequence or expression-based qualities of the pre-miRNA sequence. Although each of these features will provide valuable information towards being able to correctly classify a pre-miRNA sequence, their performance is limited due to focusing on only one line of evidence. A new approach which makes use of the strengths of each of these techniques could therefore enhance miRNA prediction. Such a classifier will take advantage of the presence of more diverse features for classification, and it is expected that features from these different views will contain less overlapping information. 
In this chapter we propose an active learning approach suitable for developing a novel highperformance miRNA prediction tool. In this method we use both genomic and transcriptome data to incorporate the advantages of de novo and NGS-based prediction methods and try to cover the deficiencies of each method. An integrated feature set comprised of both sequence and expression-based features is used for better classification, and by reducing the number of labeled instances required in the training process, the overall cost of miRNA prediction is minimized. We also aim to identify an active learning approach that will identify and label those instances that will maximize performance increase of the classifier. In this chapter, we intend to improve the performance of current state-of-the-art of miRNA prediction methods using active learning, and compare our results with various learning strategies to evaluate the potential of applying active learning to miRNA prediction.

\subsection{Methods}

Since performing actual wet-lab experiments to validate novel miRNA predictions falls outside of the scope of this thesis, the process of active learning is here simulated using high confidence labelled data. We begin with a large set of labelled data. We then simulate the case where only a small subset of the data is labelled (forming our seed training set and

independent test set), whereas the majority of data are simulated to be unlabelled (forming our active set). During active learning, when a sample is selected for labelling by wet-lab experiments, we simulate those experiments by simply looking up the true known label for that sample.

To successfully apply active learning to miRNA classification, a data set must first be assembled including high-confidence true positive and negative instances for training and testing. The size and composition of this data set is important since the ratio of negative to positive instances affects the class imbalance. In addition, some methods may be sensitive 
to the size of the initial seed training set. Next, feature selection must be applied to identify the appropriate features for training the classifier. The choice of classifier is also important, as there is no a single algorithm which is always guaranteed to perform better than others. After the framework is setup, the number of active learning iterations to perform and the number of newly labeled instances to add at each iteration must also be considered. The general framework of our proposed classifier is presented in Figure 11.

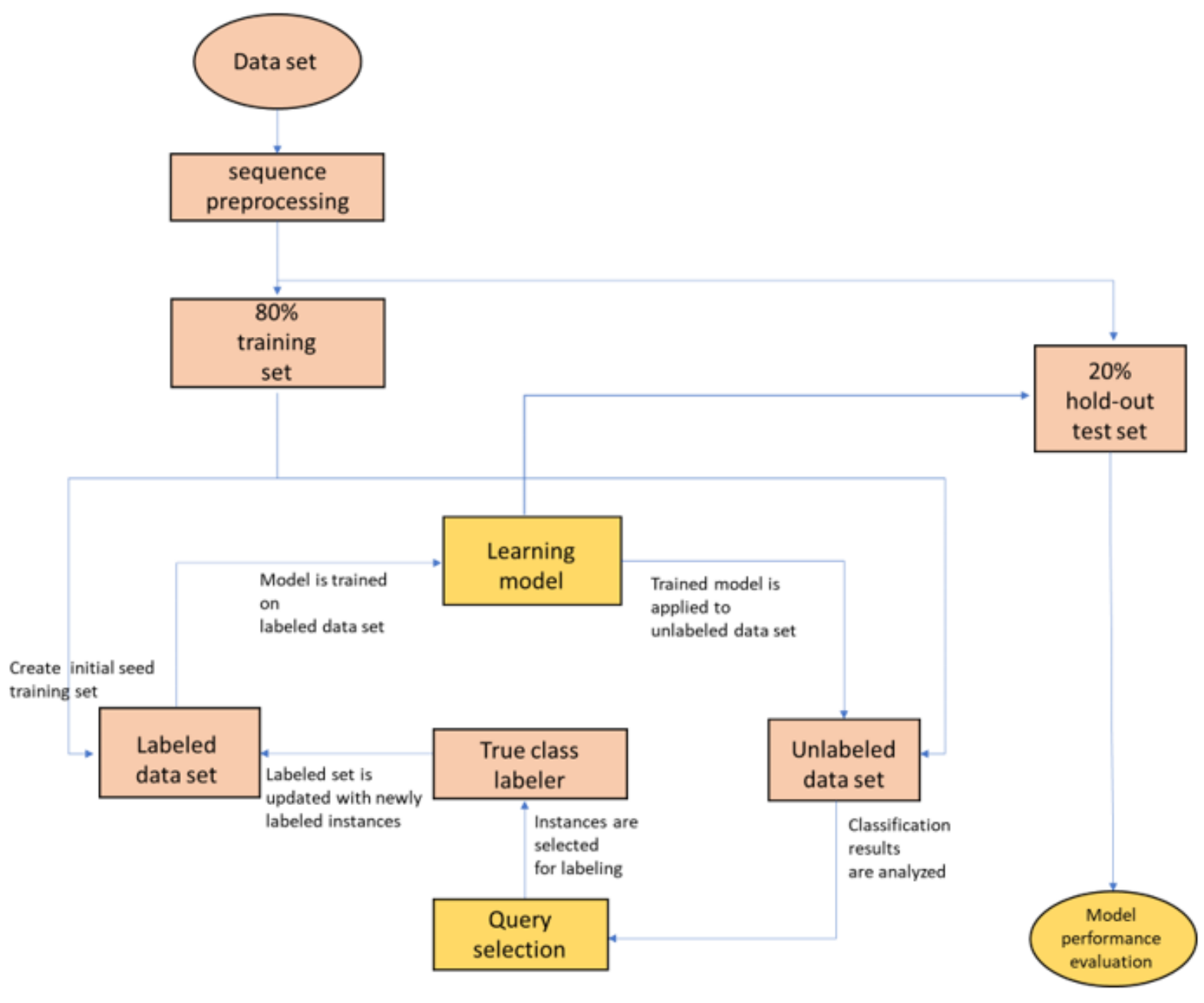

Figure 11. Active learning miRNA classification framework

Feature selection is applied to identify an optimal feature set for the training of the classifiers. As mentioned in Section 3.3, 215 sequenced-based and 8 expression-based features are derived in total from all the data sets. We use a method similar to the one 
proposed by [12] to maximize the performance of the classifier. This method consists of integrating the different lines of evidence to create a more robust feature set. Therefore, we create an integrated feature set comprising both expression and sequence-based features. According to previous work, it is expected that this integrated classifier will show an enhanced performance $[12,68]$. However, using all 223 features would be computationally expensive and may be unnecessary. In order to obtain the most informative features, the correlation-based feature subset selection method in the Weka package [94] is applied to all the data from the six species. This algorithm selects features based on the lack of intercorrelation between them and their predictive ability. To obtain a single feature set with a good generalization across all species, the entire training set (representing $80 \%$ of the total data) from all six species is used. The algorithm results in a total of 19 features being identified as the strongest predictors with the lowest inter-correlation between them. From the 19 features, 6 expression-based and 13 sequence-based features are chosen by the algorithm. This integrated feature set is used for all experiments conducted in this chapter.

Our proposed active learning algorithm is applied to 6 different species, as specified in Table 2 in Section 3.2. Six active learning models are built for these six species in a similar way to ensure the consistency of our results. Positive and negative labeled instances are collected as described in Section 3.2. After removing redundant sequences and obtaining the final labeled set, an initial split of the data is applied. For each species, the data is split into $20 \%$ hold out data, which is used for testing the performance of the classifier, and $80 \%$ which is used for training the classifier.

\subsubsection{Selecting Size of Initial Seed Training Set}

The size of the initial seed training set is an important factor in performance evaluation, as a large training set would provide sufficient data to create a strong classifier in the initial iteration of training and negating the need for active learning to add more training samples. 
Therefore, the existence of too many labeled instances for training in the first iteration will result in a classifier that has already achieved a high performance and allows little room for improvement [77]. We therefore ran an experiment on the human species data set to determine the ideal size of the initial training set. Random forest classifiers were used for testing with all parameters set to the default of the SKLearn random forest library [93] and the number of trees set to 500 . Random seeds were selected from the $80 \%$ split as the training data set to train random forest classifiers. The number of labeled instances were incrementally increased, starting from 5 positive and 5 negative instances and adding 5 to each class until reaching 50 labeled instances in each class. This procedure was repeated 100 times using different seed samples. This was done to see how the classifier performs using different numbers of labeled instances. The results for these experiments can be viewed in Table 3. As it can be seen, when increasing the training set size from 10 to 20, the classifier's performance undergoes a large increase. Further increases in training set size do not exhibit such strong increases in classification accuracy, indicating that the classifier performance quickly saturates with increasing training set size. We therefore decided to use 5 positive and 5 negative labeled instances for our initial seed training set to use fewer labeled instances and to allow room for improvement through active learning.

\begin{tabular}{|c|c|c|}
\hline $\begin{array}{c}\text { \# of } \\
\text { instances in } \\
\text { training set }\end{array}$ & $\begin{array}{c}\text { Classifier's average area } \\
\text { under precision-recall curve }\end{array}$ & $\begin{array}{c}\text { Classifier's average area } \\
\text { under ROC curve }\end{array}$ \\
\hline $\mathbf{1 0}$ & $0.6964( \pm 0.022)$ & $0.8280( \pm 0.020)$ \\
\hline $\mathbf{2 0}$ & $0.7786( \pm 0.011)$ & $0.8639( \pm 0.010)$ \\
\hline $\mathbf{3 0}$ & $0.7901( \pm 0.007)$ & $0.8816( \pm 0.007)$ \\
\hline $\mathbf{4 0}$ & $0.8083( \pm 0.007)$ & $0.8871( \pm 0.007)$ \\
\hline $\mathbf{5 0}$ & $0.8239( \pm 0.005)$ & $0.8953( \pm 0.005)$ \\
\hline $\mathbf{6 0}$ & $0.8333( \pm 0.005)$ & $0.9080( \pm 0.004)$ \\
\hline $\mathbf{7 0}$ & $0.8350( \pm 0.004)$ & $0.9099( \pm 0.004)$ \\
\hline $\mathbf{8 0}$ & $0.8375( \pm 0.004)$ & $0.9146( \pm 0.004)$ \\
\hline $\mathbf{9 0}$ & $0.8446( \pm 0.004)$ & $0.9122( \pm 0.004)$ \\
\hline $\mathbf{1 0 0}$ & $0.8586( \pm 0.004)$ & $0.9185( \pm 0.004)$ \\
\hline
\end{tabular}

Table 3. Average area under ROC and precision-recall curves for classifier using different training set sizes 


\subsubsection{Selection of Classifier Type}

Classifier selection is also done through experimentation, similar to determining the initial training set size. It has been proposed that SVM generally shows optimal performance in an active learning setup [80], and some experiments have compared SVM in active learning against other methods and have approved this theory $[79,83]$. Experiments performed in the field of miRNA classification have also suggested that SVM generally performs well when used for miRNA classification $[55,58]$. Others have suggested that random forest results in a better balance between sensitivity and specificity for miRNA prediction[10, 64, 65], and applying active learning using decision trees has also shown effective [81]. Therefore, we decided to test both methods to identify the best classifier for our problem. To do so, SVM and random forest classifiers were tested on our human data set.

To compare the performance of the two classifiers, two active learning frameworks were developed. For the SVM classifier, a linear kernel classifier with all other parameters set to the default of the SKLearn SVM library [93] was developed. The random forest classifier was also built using the default parameters of the SKLearn random forest library [93], with the number of trees set to 500 . The training set of each classifier consists of the same 5 positive and 5 negative labeled samples, and the integrated feature set previously obtained is used for classification. The $80 \%$ split of the data is used for training and $20 \%$ is used for performance evaluation. At each iteration, the classifiers are trained based on the training data, and are then applied to the remaining $80 \%$ of the data for prediction scores. The labels for the $80 \%$ split have been held back and are used whenever a prediction is queried. The hold-out $20 \%$ data is used for performance estimation at each iteration. A self-learning strategy is used for this comparison, whereby the most confident positive and negative predicted instances are added to the training set of the classifier at each iteration without being queried for their true class. Six iterations are performed in this manner to compare 
the two classifiers and choose the best performing one to use for all experiments. The results of these experiments can be viewed in Table 4. As it can be observed, the random forest classifier outperforms the SVM classifier using the same samples. Considering these results, we therefore used random forest classifiers for all subsequent experiments.

\begin{tabular}{|c|c|c|}
\hline Iteration & $\begin{array}{c}\text { SVM classifier's } \\
\text { AUPRC }\end{array}$ & $\begin{array}{c}\text { RF classifier's } \\
\text { AUPRC }\end{array}$ \\
\hline $\mathbf{1}$ & $0.6792( \pm 0.023)$ & $0.6964( \pm 0.020)$ \\
\hline $\mathbf{2}$ & $0.7880( \pm 0.020)$ & $0.8159( \pm 0.022)$ \\
\hline $\mathbf{3}$ & $0.7619( \pm 0.013)$ & $0.7951( \pm 0.013)$ \\
\hline $\mathbf{4}$ & $0.7265( \pm 0.014)$ & $0.7998( \pm 0.015)$ \\
\hline $\mathbf{5}$ & $0.7985( \pm 0.015)$ & $0.8190( \pm 0.012)$ \\
\hline $\mathbf{6}$ & $0.8071( \pm 0.013)$ & $0.8147( \pm 0.014)$ \\
\hline
\end{tabular}

Table 4. Comparison of the area under precision-recall for active learning using SVM and random forest classifiers on hsa data set. Means \pm standard deviations are shown, representing 100 repetitions of each experiment.

\subsubsection{Selecting Stopping Criterion}

To determine when to stop active learning, we decided to follow the suggestion of Lewis and Gale [7]. In this strategy, labeling will stop once it is determined that the classifier has reached its maximum performance effectiveness. The effectiveness of the classifier is evaluated by testing its performance at each iteration on an independent labeled data set with preserved labels. This data set consists of all the instances in the $20 \%$ split of the data. All labels in this data set are held back and only used for evaluation. We record the AUPRC at each iteration to construct the learning curve of the classifier to view its estimated performance. Once maximum effectiveness is reached and the new iterations do not significantly increase performance, the learning and annotation process will be stopped. When this procedure was applied to our data sets, it was found that in most cases maximum performance effectiveness is reached around the $11^{\text {th }}$ iteration. Figure 12 demonstrates this fact for the human data set as an example, where it can be seen that 
performance remains relatively constant towards the final iterations. Therefore, we set the number of learning iterations to 11 for all experiments. More practically, often active learning is stopped when annotation resources are exhausted. In the present thesis, all experiments were run for 11 iterations. For some of the experiments, use of a dynamic stopping criterion may have resulted in slightly earlier or later stopping than this point.

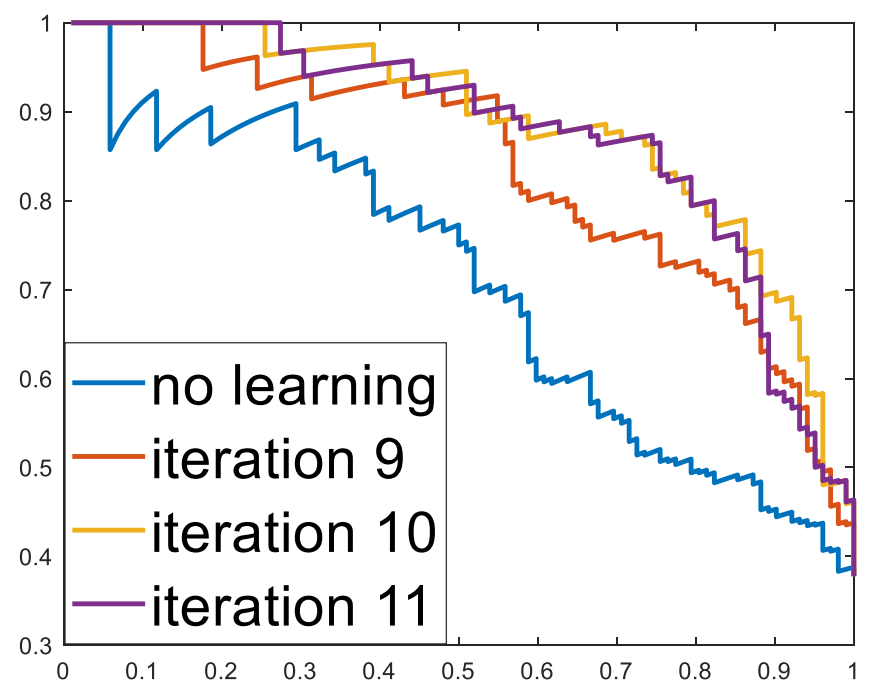

Figure 12. Average precision-recall curve for human data set at specific iterations. The yaxis represents precision while the $\mathbf{x}$-axis is recall.

\subsubsection{Active Learning Strategy}

Another decision that needs to be made for an active learning experiment setup is which points to query to reach the classifier's maximum performance and how many new samples to label in each iteration. In uncertainty query strategies, the instances which the classifier is least confident of in its prediction are queried $[37,38]$. In this strategy, the two most uncertain points will be chosen from the $80 \%$ unlabelled data for annotation. These points will either be those with a $50 \%$ percent chance of being in the positive or negative class, or those closest to the decision boundary. In the Certainty-based Query Strategy, the two most confident points (one positive, one negative) will be chosen from the $80 \%$ unlabeled 
data set for annotation. These instances will be the classifier's most confident predictions, with a prediction score close to $100 \%$ for one of the classes. Both of these strategies are tested on all the experiments in order to determine which query strategy is more effective. The active learning strategy which provides an improved performance using similar data will be the more effective strategy.

\subsubsection{Summary of Methods}

As a summary, our main classifier is built using a random forest classifier with 500 trees and the default parameters of the SKLearn random forest library [93], and an initial seed training set of 10 labeled samples (5 positive, 5 negative) randomly selected from the $80 \%$ split. The initial model is built on this seed training set using the integrated feature set. After the model is built, it is applied to the $80 \%$ unlabeled set (minus the initial training set). Depending on the query strategy, either the most or least confident positive and negative predictions will be selected for annotation. Once the true classes of the samples are determined, they will be removed from the unlabeled set and added to the training set, and this procedure will continue throughout the iterations. In a real-life experiment, obtaining the true class of an instance would require wet-lab experiments and is therefore a lengthy and costly process. In our case however, all labels are available but withheld, therefore our query process only required looking up the true class of the instance. After each iteration, the model is re-trained on the new training set and the performance of the classifier is noted in the learning curve of the classifier. The trends of the learning curve is studied, and the learning process is stopped once it is observed that maximum effectiveness is reached and performing extra iterations does not result in performance increase.

We applied our proposed active learning strategy to the data sets pertaining to six distinct species as described in section 3.2. Both certainty and uncertainty query strategies were applied to each data set to compare the results and identify the best strategy for this 
problem. In addition, a self-training (self-learning) strategy was also implemented to the data sets. The self-training strategy used in these experiments is built using the same setup used for the active learning strategies. We used the same random forest classifier, integrated feature set and training and test sets as the active learning classifiers. In this approach, we do not query the true labels of the instances at each iteration and add samples to the training set by assuming that the predicted label is correct. To further explain, at each iteration, the random forest built using the integrated feature set and training data will be applied to the $80 \%$ unlabeled set. The two instances that the classifier most confidently predicts as a positive and negative will be labeled according to the prediction and added directly to the training set without being checked for their true label. This process is repeated for the same number of iterations as the active learning strategy to compare the performances on the final training set using the same number of training instances. A passive learning classifier is also constructed following a nearly identical approach. The only difference in the passive classifier is that, at each iteration, two instances are randomly selected from the unlabeled data to be labelled by the oracle and added to the training data.

The final performance of all methods are plotted on a precision-recall curve, and the performance at each iteration is reported for the certainty and uncertainty query strategies, passive learning and self-training. The "no learning" classifier demonstrates classification on the initial training set before any learning iteration is applied. The classifier's performance at each iteration is evaluated by calculating the area under the precision-recall and ROC curves. Prevalence-corrected precision is calculated instead of precision in all experiments to compensate the fact that the experiment sets may not accurately reflect the true class imbalance. This distribution of data is randomly repeated 100 times, resulting in different seed training sets, test sets and unlabeled sets. Therefore, each learning strategy is evaluated 100 times using different initial seed training sets. In the next section, we will 
observe the results of our proposed active learning method and will compare the results of certainty and uncertainty-based query strategies as well as passive learning and no active learning.

\subsection{Results}

We first compare the two different active learning strategies, uncertainty- vs. certaintybased learning. Next, active learning is compared with passive- and self-learning strategies, where the latter two represent two different baseline methods. Lastly, we demonstrate that using an integrated feature set outperforms classifiers trained solely using sequence- or expression-based features, and show that active learning further improves the integrated classifier's performance.

\subsubsection{Comparing Uncertainty- and Certainty-based Active Learning}

To demonstrate the results of our active learning strategies, we have included a table reporting performance at each iteration for the human species. To confirm our results, we also applied the same method to the other five species and have included the resulting tables in Appendix A of this thesis. The results show the performance of the classifiers using area under precision-recall and ROC curves at each iteration. A total of 11 iterations are performed for each strategy, resulting in a total of 22 instances being added to the initial training set. Therefore, the results on the last iteration show the performance of a classifier trained using 32 labeled instances. Table 5 shows the results obtained at each iteration by applying our certainty and uncertainty-based active learning query strategy to the human data set. As expected, a trend of increasing performance with increasing training set size is observed; however, this increase in performance is not uniformly monotonic. 


\begin{tabular}{|c|c|c|c|c|}
\hline $\begin{array}{c}\text { Active } \\
\text { learning } \\
\text { iteration }\end{array}$ & $\begin{array}{c}\text { certainty-based } \\
\text { classifier's } \\
\text { average AUPRC }\end{array}$ & $\begin{array}{c}\text { uncertainty- } \\
\text { based } \\
\text { classifier's } \\
\text { average AUPRC }\end{array}$ & $\begin{array}{c}\text { certainty-based } \\
\text { classifier's } \\
\text { average AUROC }\end{array}$ & $\begin{array}{c}\text { uncertainty- } \\
\text { based } \\
\text { classifier's } \\
\text { average AUROC }\end{array}$ \\
\hline $\mathbf{1}$ & $0.696( \pm 0.02)$ & $0.696( \pm 0.02)$ & $0.765( \pm 0.02)$ & $0.765( \pm 0.02)$ \\
\hline $\mathbf{1}$ & $0.795( \pm 0.01)$ & $0.701( \pm 0.01)$ & $0.850( \pm 0.02)$ & $0.774( \pm 0.01)$ \\
\hline $\mathbf{3}$ & $0.794( \pm 0.01)$ & $0.810( \pm 0.01)$ & $0.846( \pm 0.01)$ & $0.874( \pm 0.01)$ \\
\hline $\mathbf{4}$ & $0.818( \pm 0.01)$ & $0.813( \pm 0.01)$ & $0.869( \pm 0.01)$ & $0.874( \pm 0.01)$ \\
\hline $\mathbf{5}$ & $0.813( \pm 0.01)$ & $0.833( \pm 0.01)$ & $0.868( \pm 0.01)$ & $0.884( \pm 0.01)$ \\
\hline $\mathbf{6}$ & $0.810( \pm 0.01)$ & $0.807( \pm 0.01)$ & $0.850( \pm 0.01)$ & $0.857( \pm 0.01)$ \\
\hline $\mathbf{7}$ & $0.800( \pm 0.01)$ & $0.832( \pm 0.01)$ & $0.850( \pm 0.01)$ & $0.872( \pm 0.01)$ \\
\hline $\mathbf{8}$ & $0.800( \pm 0.01)$ & $0.839( \pm 0.01)$ & $0.857( \pm 0.01)$ & $0.894( \pm 0.01)$ \\
\hline $\mathbf{9}$ & $0.812( \pm 0.01)$ & $0.840( \pm 0.01)$ & $0.861( \pm 0.01)$ & $0.897( \pm 0.01)$ \\
\hline $\mathbf{1 0}$ & $0.822( \pm 0.01)$ & $0.829( \pm 0.01)$ & $0.866( \pm 0.01)$ & $0.884( \pm 0.01)$ \\
\hline $\mathbf{1 1}$ & $0.817( \pm 0.01)$ & $0.867( \pm 0.01)$ & $0.869( \pm 0.01)$ & $0.906( \pm 0.01)$ \\
\hline & $0.797( \pm 0.01)$ & $0.875( \pm 0.01)$ & $0.830( \pm 0.01)$ & $0.917( \pm 0.01)$ \\
\hline
\end{tabular}

Table 5. Certainty and uncertainty-based active learning query strategy results for human data set. Values represent the mean ( \pm standard deviation) of 100 repetitions with different data splits.

Figure 13 is constructed using the data from Table 5, in addition to results for the other species. This figure illustrates the performance of the certainty and uncertainty-based active learning query strategies at each iteration for the six data sets, in addition to the same results for passive and self-learning classifiers. From the plots, it is evident that both active learning strategies clearly outperform the other two classifiers over five of the six data sets. For the human data set, 509 positive and 842 negative labeled instances are available in total. By applying the initial data split, $20 \%$ of the data is held back for performance evaluation, meaning the model is tested on 102 positive and 168 negatives at each iteration. As it can be observed, after 11 iterations of learning, the certainty-based classifier increases the classifier's average AUPRC by $14.4 \%$ and its AUROC by $8.49 \%$. There is a large increase in performance after the first iteration and, during the next iterations, small increases are observed. After the same number of iterations, the uncertainty-based classifier increases average AUPRC by $25.7 \%$ and AUROC by $19.9 \%$. Performance results in the uncertainty-based strategy show a monotonic increasing trend throughout the iterations, resulting in a significantly higher performance increase. 
hsa

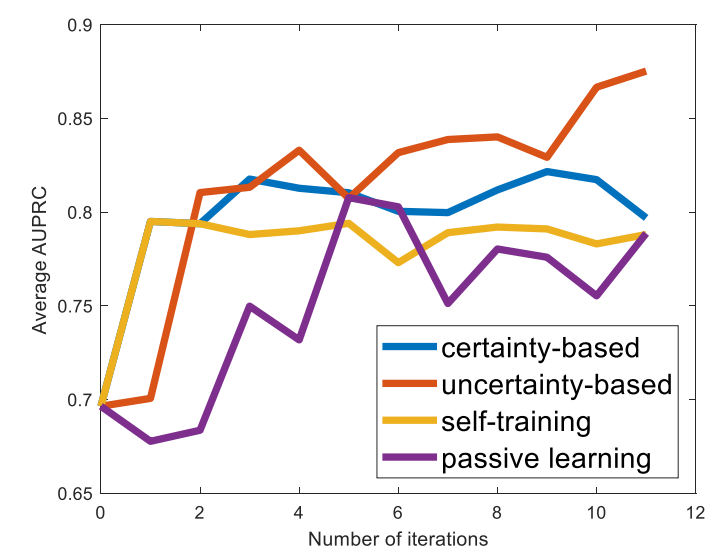

dme

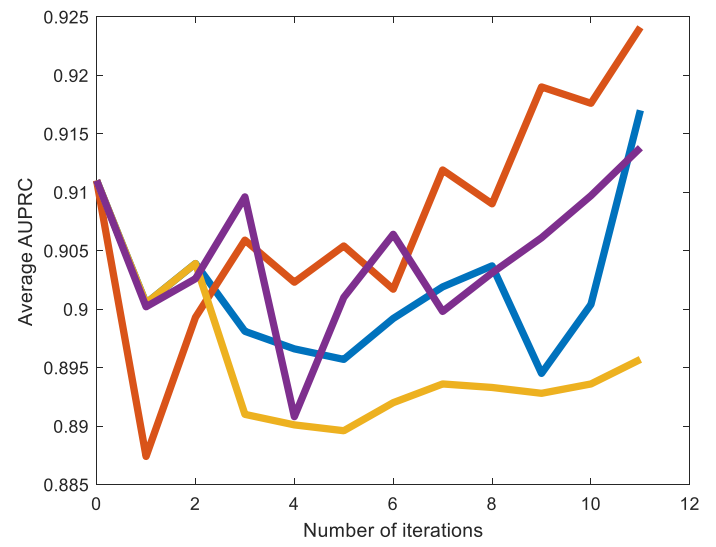

gga

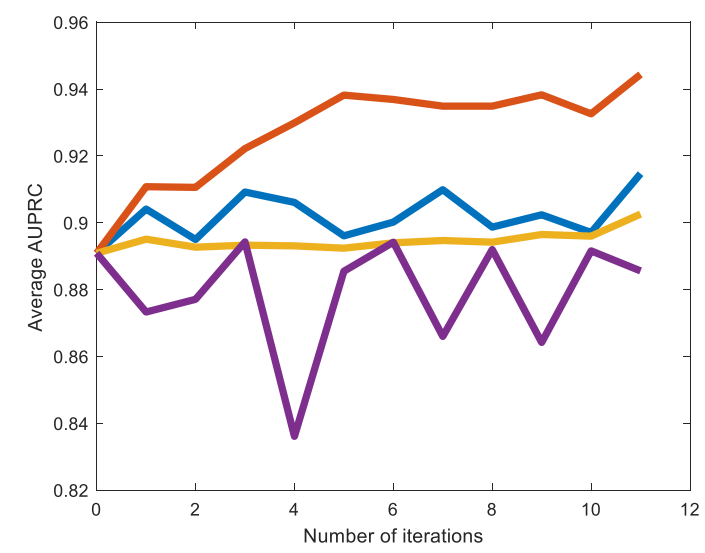

$m m u$

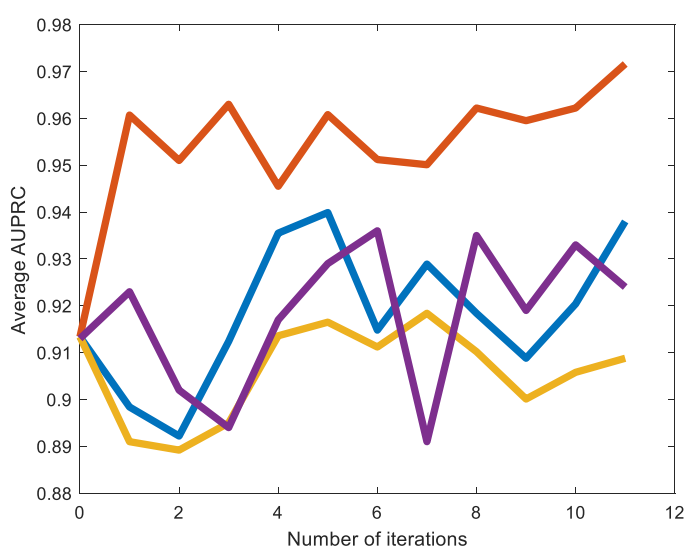

bta

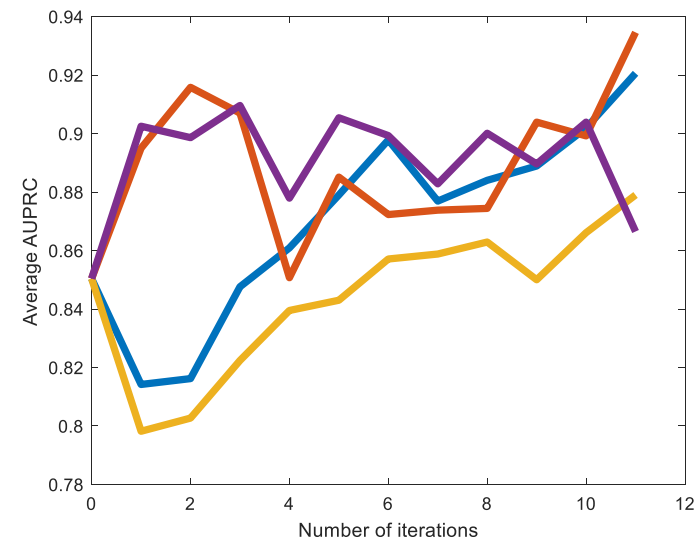

eca

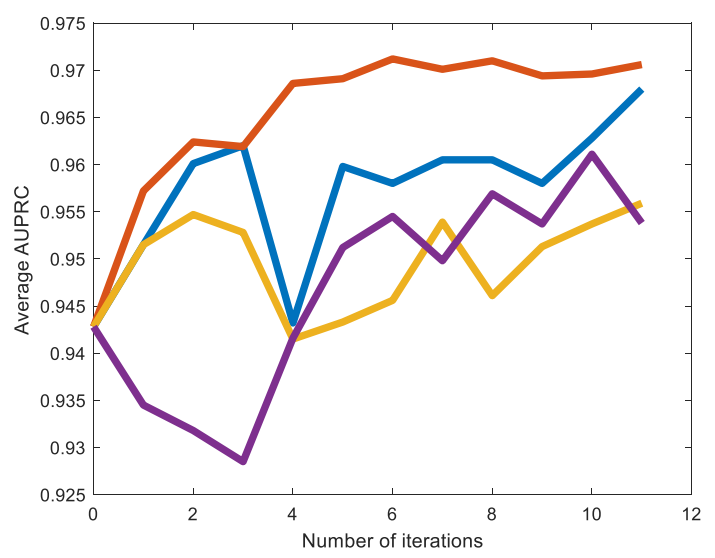

Figure 13. Certainty and uncertainty-based active learning query strategy results vs. passive and self-learning for all six data sets over 11 iterations. 
When tested on the mouse data set containing 73 positive and 169 negative instances, after 11 learning iterations, the certainty-based classifier increases average AUPRC by $2.70 \%$ and AUROC by $2.04 \%$. The uncertainty-based classifier increases AUPRC by $6.37 \%$ and AUROC by $4.02 \%$. When tested on the fruit-fly data set consisting of 22 positive and 19 negative instances, the certainty-based classifier increases average AUPRC by $0.65 \%$ and it's AUROC by $1.02 \%$ after 11 iterations are performed. The uncertainty-based classifier increases AUPRC by $1.44 \%$ and AUROC by $1.51 \%$. Both learning methods show a high level of fluctuation within their results during the different iterations, and a drop is observed in the performance after the start of the learning iterations. After around six iterations, both methods recover from the performance drop and slowly approach better results during the last iterations, especially the uncertainty-based classifier.

The performance of the two active learning strategies over the cow data set (bta) is measured over a test set of 66 positive and 130 negative instances. The certainty-based classifier demonstrates an 8.25\% improvement in the average AUPRC, and 3.05\% improvement in average AUROC after 11 iterations. The uncertainty-based classifier increases the average AUPRC and AUROC by $9.90 \%$ and $5.04 \%$, respectively. On this data set, passive learning performs unusually well and nearly matches the performance of active learning over all iterations save the last. The chicken test set consists of 39 positive and 21 negative instances, and by applying 11 iterations of the certainty-based classifier to this test set, average AUPRC and AUROC are boosted by $2.67 \%$ and $1.97 \%$, respectively. The uncertainty-based strategy increases AUPRC by $6.00 \%$ and AUROC by $9.00 \%$ once it is applied for 11 iterations. The uncertainty-based classifier shows less fluctuation and a more monotonic trend compared to the certainty-based classifier. The certainty-based classifier applied to the horse test set containing 73 positive and 46 negative instances increases average AUPRC by $2.67 \%$ and AUROC by $1.42 \%$ after 11 iterations are performed. The uncertainty-based classifier increases AUPRC by $2.95 \%$ and AUROC by $1.28 \%$. A relatively 
monotonic increasing trend is observed in performance over both strategies for the horse data set, although some fluctuations are observed after the seventh iteration, which may suggest the maximum effectiveness has been reached.

\subsubsection{Passive vs. Active Learning}

Table 6 compares the final result of classification using certainty and uncertainty-based active learning strategies, as well as passive, self-learning, and the initially trained classifier ("no learning") for all six species. From the results, it is evident that both active learning strategies perform better than the passive learning classifier on each of the experiments, yielding a higher average performance. Considering the "no learning" classifier as the baseline performance, the uncertainty-based classifier demonstrates the best increase in performance amongst the four classifiers, increasing the average performance amongst all six species by $8.72 \%$. The certainty-based classifier comes in second with an average improvement of $5.23 \%$, followed by the passive learning and self-training classifiers that surpasses the "no learning" classifier by $2.86 \%$ and $2.83 \%$, respectively. The maximum performance increase produced by the uncertainty-based strategy is $25.7 \%$ ( $h s a$ ), where that maximum drops to $14.4 \%, 13.24 \%$ and $13.11 \%$ for the certainty-based, passive and self-learning classifiers, respectively. In addition to performing worse than the two active learning strategies on all data sets, the self-training classifier actually performs worse than the original "no learning" classifier on the mouse and fruit-fly data sets, decreasing average AUPRC by $0.50 \%$ and $1.68 \%$, respectively. The same occurs for the passive learning classifier on the chicken data set. The performance of the methods as given in Table 6 on both the independent data sets and on average suggest that the uncertainty-based active learning strategy is a better approach for miRNA classification compared to the other three methods. These results represent the mean performance over 100 repetitions of the 
experiment. For clarity, the standard deviations are not shown; however, they are included in Table A6 in Appendix A.

\begin{tabular}{|c|c|c|c|c|c|}
\hline Data set & $\begin{array}{l}\text { No } \\
\text { learning } \\
\text { average } \\
\text { AUPRC }\end{array}$ & $\begin{array}{l}\text { Self-training } \\
\text { average AUPRC }\end{array}$ & $\begin{array}{l}\text { Passive learning } \\
\text { average AUPRC }\end{array}$ & $\begin{array}{l}\text { Certainty-based } \\
\text { active learning } \\
\text { average AUPRC }\end{array}$ & $\begin{array}{l}\text { Uncertainty- } \\
\text { based active } \\
\text { learning average } \\
\text { AUPRC }\end{array}$ \\
\hline $\boldsymbol{h s a}$ & 0.696 & $0.788(+13.1 \%)$ & $0.789(+13.2 \%)$ & $0.797(+14.4 \%)$ & $0.875(+25.7 \%)$ \\
\hline $\boldsymbol{m m u}$ & 0.913 & $0.909(-0.50 \%)$ & $0.924(+1.16 \%)$ & $0.938(+2.69 \%)$ & $0.972(+6.37 \%)$ \\
\hline $\boldsymbol{d m e}$ & 0.911 & $0.896(-1.68 \%)$ & $0.914(+0.30 \%)$ & $0.917(+0.66 \%)$ & $0.924(+1.44 \%)$ \\
\hline $\boldsymbol{b t a}$ & 0.850 & $0.879(+3.36 \%)$ & $0.867(+1.89 \%)$ & $0.921(+8.25 \%)$ & $0.935(+9.90 \%)$ \\
\hline $\boldsymbol{g g a}$ & 0.891 & $0.903(+1.31 \%)$ & $0.886(-0.60 \%)$ & $0.915(+2.67 \%)$ & $0.944(+6.01 \%)$ \\
\hline eca & 0.943 & $0.956(+1.39 \%)$ & $0.954(+1.17 \%)$ & $0.968(+2.67 \%)$ & $0.971(+2.95 \%)$ \\
\hline $\begin{array}{c}\text { Avg. } \\
\text { increase }\end{array}$ & - & $+2.83 \%$ & $+2.86 \%$ & $+5.23 \%$ & $+8.72 \%$ \\
\hline
\end{tabular}

Table 6. Final classification results using certainty and uncertainty-based active learning strategies, passive training, self-learning and "no learning" for all six species. Percentages change relative to "no learning" classifier is shown in parentheses.

Figure 14 compares the final performance curves of the two active learning strategies against passive learning, self-training and no learning for all the six data sets. The active learning strategies outperform the other two strategies over all data sets. These results demonstrate how the two characteristics of active learning, being the ability to query points and to select which points to query, give it an advantage over the other methods. In the self-training method, although only the most confident samples are selected, they cannot be queried for their true class and are added to the training set based on their predicted labels. This poses the threat of adding mislabeled points to the training data, and clearly has a negative impact on performance. In passive learning, although instances are queried before addition to the training set, they are selected randomly. The fact that randomly selected instances are not capable of increasing performance by a margin similar to that of our two active learning strategies despite enlarging the training set by the same size, demonstrates the importance of the studied selection of instance annotation in active learning. 
Overall, the results in Table 6 and Figures 13-14 demonstrate that the uncertainty-based active learning classifier outperforms the certainty-based active learning classifier and the passive and self-learning classifiers. This can be said because improved performance was observed from this classifier on all the six data sets compared to the other two methods. The experimental setup used for all three methods was consistent, all undergoing 11 iterations of learning, using the same feature sets and training and test sets. Therefore, it can be concluded that an uncertainty-based active learning strategy is the best approach for miRNA classification amongst the four tested methods. 

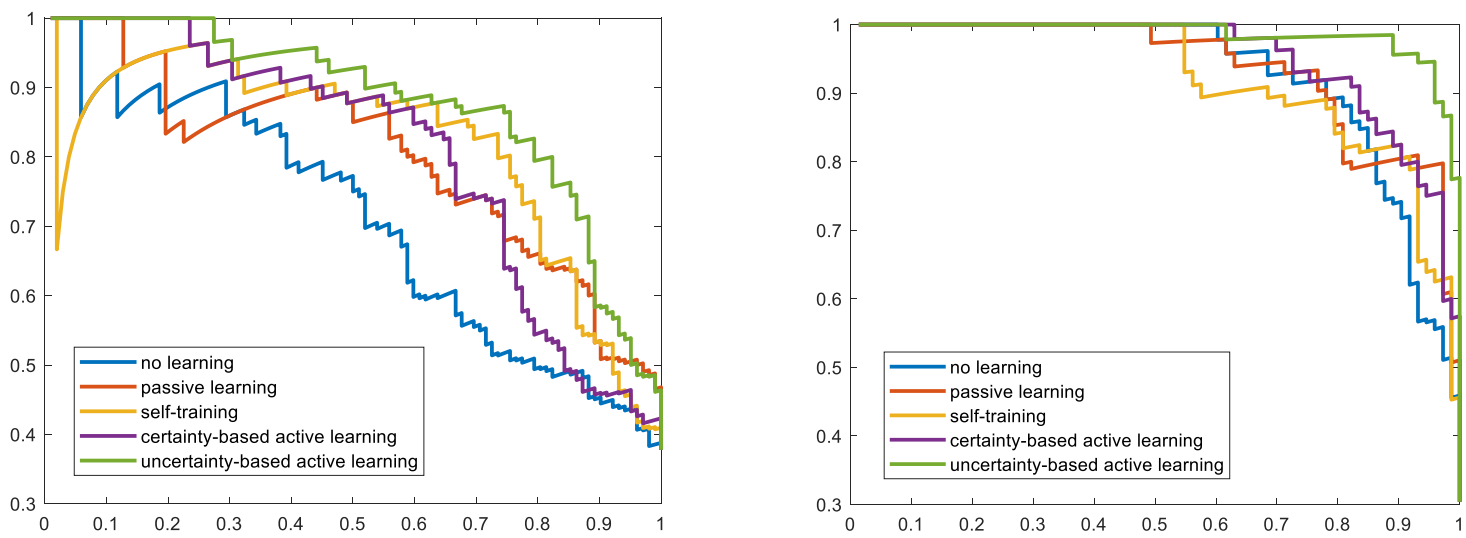

dme

bta
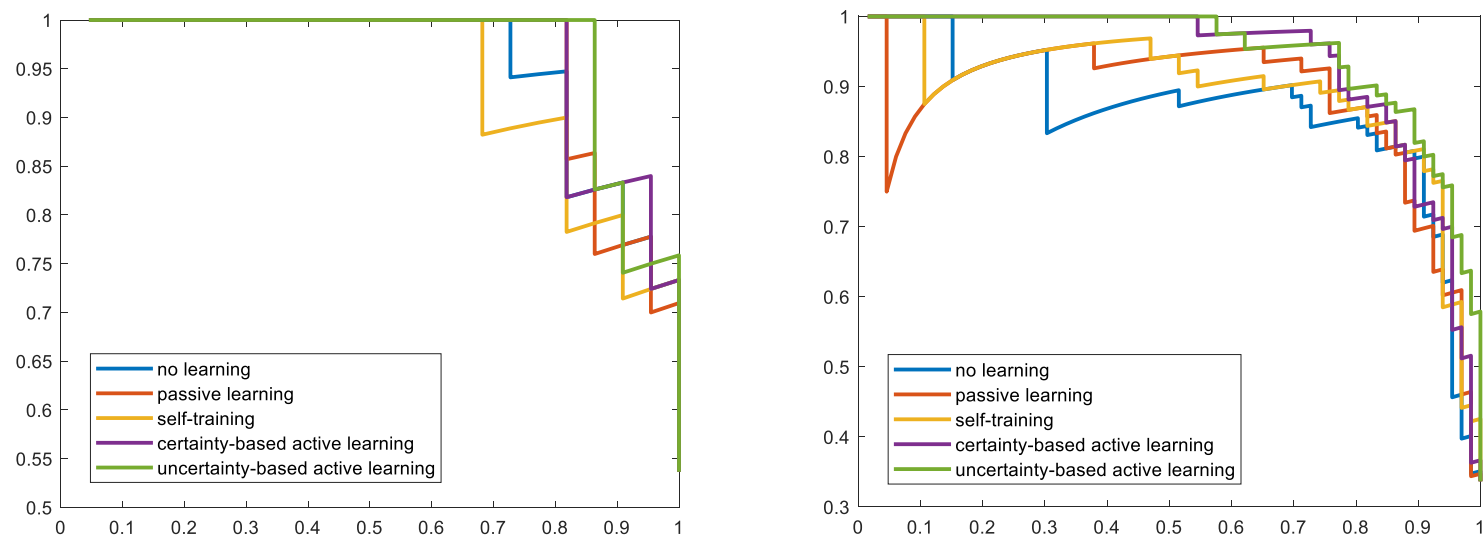

gga

eca
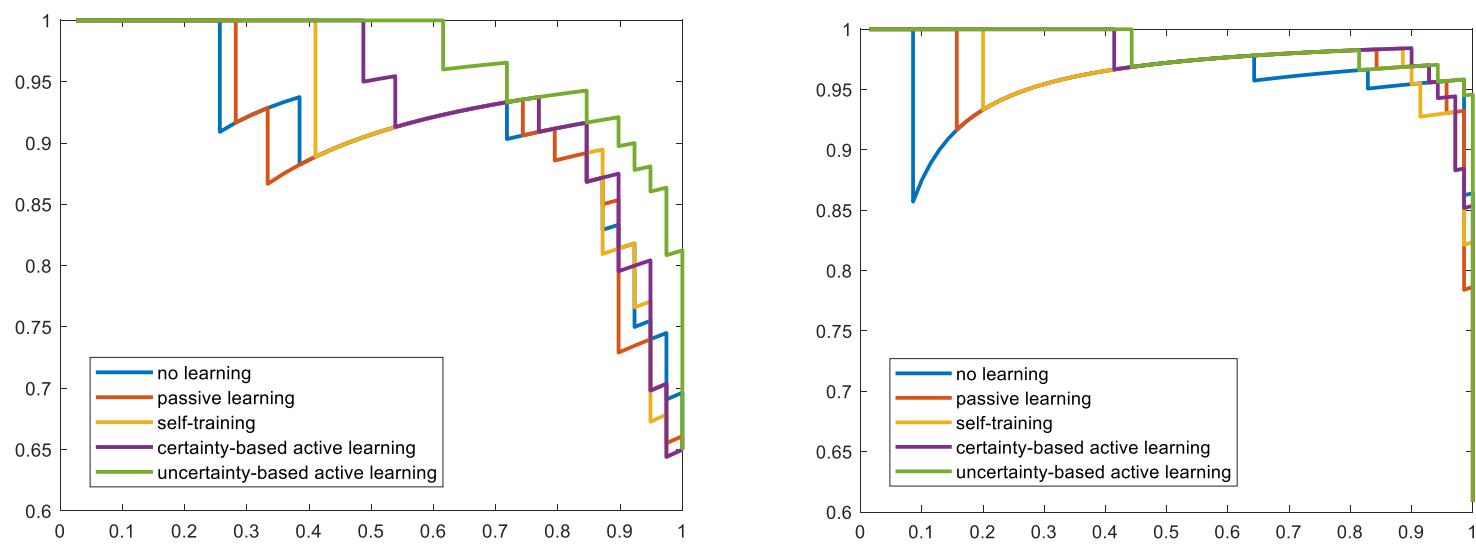

Figure 14. Precision-recall curves for all six species after completing certainty and uncertainty-based active learning, passive learning, self-training and no learning. In all plots, the $y$-axis represents precision while the $x$-axis is recall. 


\subsubsection{Benefits of an Integrated Feature Set}

We have thus far established that active learning using the uncertainty-based query strategy leads to improved performance compared to baseline methods. We here examine the benefit of using an integrated feature set vs. classifiers trained using strictly sequenceor expression-based features. Three classifiers are built using 32 randomly selected training exemplars: only sequence-based features, only expression-based features, an integrated set of both sequence- and expression-based features. Note that this is equivalent to the passive learning strategy described above, after 11 iterations. Note also that the integrated classifier is equivalent to the miPIE method, when constrained to 32 training exemplars. We dedicated the same labeling budget to all of the other methods to perform a fair comparison, and all tests were repeated 100 times. In each repetition, the splitting of data into seed training set and unlabeled set is re-randomized.

In addition, a comparison was made with the popular miRNA prediction tool miRDeep2. We have chosen to compare our method against miRDeep2 since, through independent evaluation on seven data sets, it was determined to be one of the most effective state-ofthe-art methods [95]. It must be noted that miRDeep2 represents a conservative benchmark, since this method is already trained on full, unrestricted training set. This pretrained method is applied once to the test set, hence, no standard deviation can be computed.

Figure 15 demonstrates the average precision-recall curves for all methods over each of the six data sets, and Table 7 compares the average AUPRC for each method on each data set. To again highlight the value of active learning, we reproduce the uncertainty-based active learning results from Table 6 in the final column of Table 7 below. From the results, it can be observed that the integrated classifier outperforms each of the individual views on all 
data sets. When looking at the average over all six species, the integrated classifier demonstrates a $3.3 \%$ and $2.5 \%$ average increase compared to the sequence-based and expression-based classifiers, respectively. This improved performance demonstrates the value of integrating both views for a combined feature set, as suggested by [68]. While making use of this integrated feature set, active learning yields more substantial increases in performance: $6.93 \%$ compared to the sequence-based classifier, $5.93 \%$ compared to the expression-based classifier, $2.99 \%$ compared to the integrated classifier, and $7.91 \%$ compared to miRDeep2. As observed from both the figure and table, active learning outperforms all the other methods on individual data sets as well as on average, which clearly demonstrates that active learning can improve classification performance using the same labeling budget. To test for statistical significance of differences between methods observed in our results, the ANOVA test was first applied to the results in Table 7 . This test indicated that there is a statistically significant difference between the mean results of the groups (at $\alpha=0.05$ ). The Tukey test was then applied between the active learning results and each of the other methods, and all results were found to be statistically significant (at $\alpha$ $=0.01$ ).

\begin{tabular}{|c|c|c|c|c|c|}
\hline $\begin{array}{c}\text { Data } \\
\text { set }\end{array}$ & $\begin{array}{c}\text { Sequence-based } \\
\text { classifier's } \\
\text { average AUPRC }\end{array}$ & $\begin{array}{c}\text { Expression- } \\
\text { based classifier's } \\
\text { average AUPRC }\end{array}$ & $\begin{array}{c}\text { Integrated } \\
\text { classifier's } \\
\text { average AUPRC }\end{array}$ & $\begin{array}{c}\text { miRDeep2 } \\
\text { classifier's } \\
\text { average AUPRC }\end{array}$ & $\begin{array}{c}\text { Active learning } \\
\text { classifier's } \\
\text { average AUPRC }\end{array}$ \\
\hline$h s a$ & $0.763( \pm 0.02)$ & $0.789( \pm 0.01)$ & $0.844( \pm 0.01)$ & 0.736 & $0.875( \pm 0.01)$ \\
\hline$m m u$ & $0.907( \pm 0.01)$ & $0.939( \pm 0.01)$ & $0.966( \pm 0.01)$ & 0.915 & $0.972( \pm 0.00)$ \\
\hline$d m e$ & $0.918( \pm 0.01)$ & $0.893( \pm 0.01)$ & $0.894( \pm 0.01)$ & 0.914 & $0.924( \pm 0.01)$ \\
\hline$b t a$ & $0.890( \pm 0.02)$ & $0.865( \pm 0.02)$ & $0.905( \pm 0.02)$ & 0.869 & $0.935( \pm 0.01)$ \\
\hline$g g a$ & $0.886( \pm 0.02)$ & $0.906( \pm 0.01)$ & $0.919( \pm 0.01)$ & 0.923 & $0.944( \pm 0.01)$ \\
\hline eca & $0.886( \pm 0.01)$ & $0.906( \pm 0.01)$ & $0.919( \pm 0.01)$ & 0.843 & $0.971( \pm 0.00)$ \\
\hline Average & 0.875 & 0.883 & 0.908 & 0.867 & 0.935 \\
\hline
\end{tabular}

Table 7. Comparing average AUPRC for all six data sets over the following methods: active learning with integrated feature set, passive sequence-based only classifier, passive expression-based only classifier, passive integrated classifier, and miRDeep2. Means \pm standard deviations are shown, representing 100 repetitions of each experiment (except for miRDeep2). 

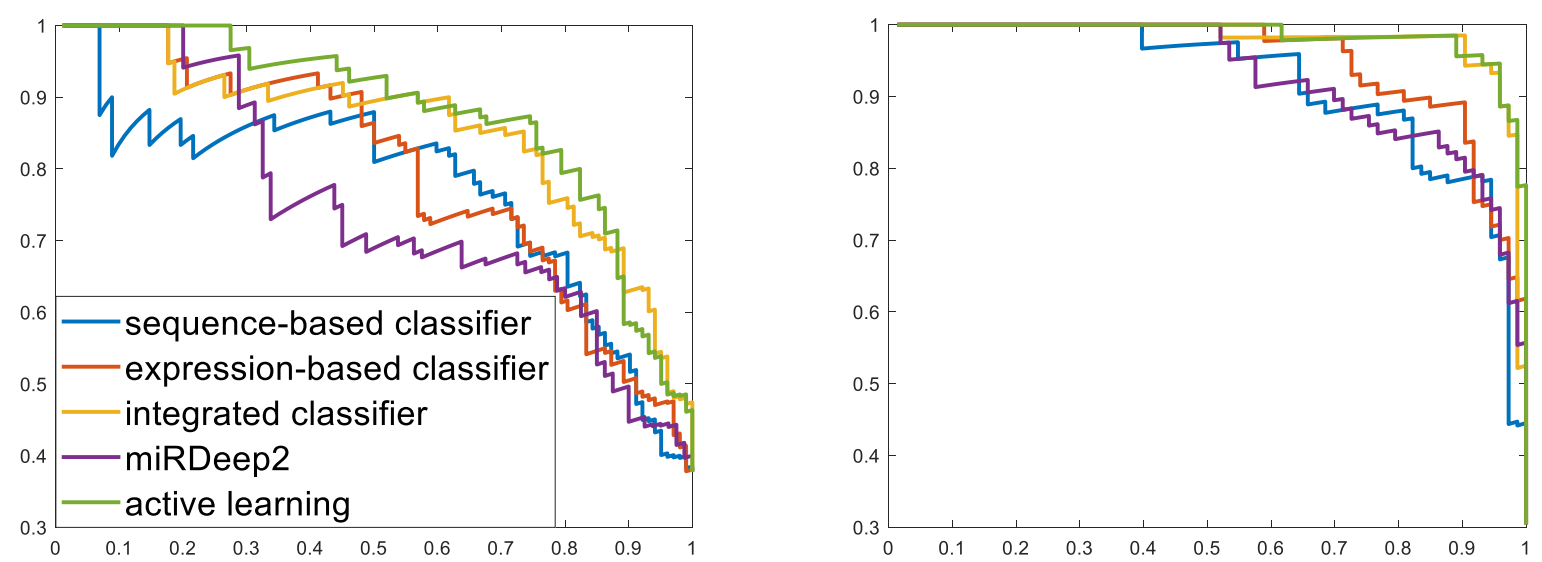

dme

bta
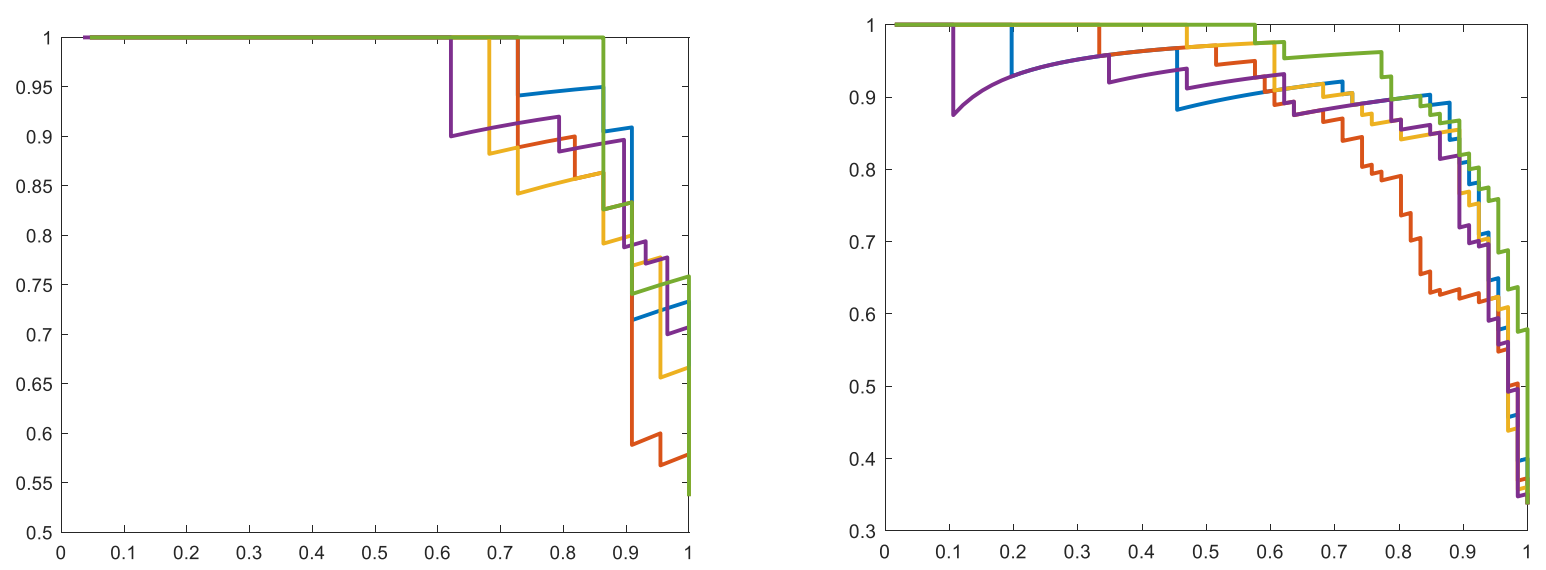

gga

eca
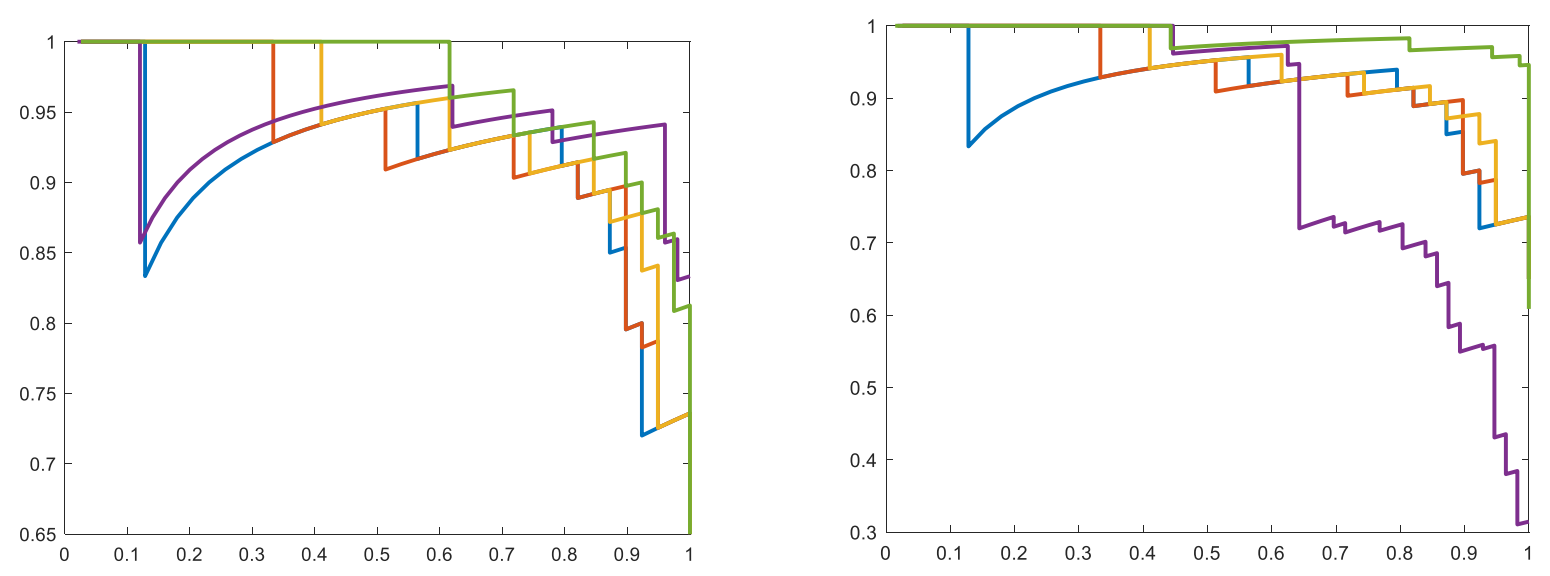

Figure 15. Comparing average precision-recall curves for all six data sets over the following methods; active learning, sequence-based only classifier, expression-based only classifier, integrated classifier, miRDeep2. In all plots, the $y$-axis represents precision while the $x$-axis is recall. 


\subsection{Discussion}

As discussed in the introduction of this chapter, determining which points to query is a significant decision in active learning. In our experiments, we created frameworks to test both certainty- and uncertainty-based query methods to compare their performances. The results presented in Table 6 show that the uncertainty-based query strategy outperforms the certainty-based strategy on all of the six data sets and has an improved average performance of $3.49 \%$ over all data sets. When looking at active learning results, it can be observed that in most data sets the certainty-based strategy shows a performance increase during the first iterations before reaching a plateau, where from that point on further iterations do not increase performance. This demonstrates that the certainty-based classifier reaches maximum effectiveness at an earlier stage compared to an uncertainty-based active learning strategy. In addition, in a species such as fruit-fly (please see Appendix A), where the average AUPRC is high to begin with, we see the certainty-based classifier increases the performance by only $0.66 \%$, whereas the uncertainty-based classifier more than doubles this increase.

The certainty-based classifier increasing performance by such a small margin when the classifier is already at a high performance, and the classifier reaching maximum effectiveness at an early stage, are both understandable consequences of the certaintybased query strategy used. When the classifier confidently predicts an instance to belong to one of the classes, it means it has sufficient information regarding that instance, extracted from samples already in the training set. Therefore, by adding this strongly predicted instance to the training set, we are just re-confirming the information the classifier had already obtained from similar instances. By considering this explanation, the reason the classifier reaches maximum effectiveness at an early stage becomes evident, as the newly added instances do not further strengthen the view. The similar explanation applies to the 
fruit-fly experiment, where by adding the top predicted points, the classifier is simply adding instances with similar characteristics to the training set. This may increase a weak classifier's performance, but if the performance is already high enough, the classifier will not have much to learn from the added instances. Both these problems can be overcome by using an uncertainty-based query approach. By using an uncertainty-based approach, we are constantly adding instances containing new information to the classifier. These instances will be the ones where the classifier had difficulty predicting, meaning it did not have sufficient information regarding their features. By adding these instances to the classifier, we help it learn their characteristics and be able to predict future similar instances with better knowledge. In this way, the uncertainty-based approach can continue to improve performance after more iterations of learning and without plateauing at an early stage.

In the self-training approach implemented in this study, we directly add the top predicted positive and negative instances to the training set in each iteration, without checking the true label. This method follows the same approach as the certainty-based, which is the addition of confident points. We previously discussed that this strategy can result in less improvement during iterations, and a faster performance plateau. In addition, the fact that this method does not check for the true label of the instances differentiates it from the previous approaches. As expected, this method proved to be weaker than both active learning strategies on average and on each of the data sets, as displayed in Table 6 . The results also show a high amount of fluctuation during iterations, demonstrating performance increase in some and decrease in others. In addition, in the mouse and fruit-fly experiments, the self-training approach performed worse than the initial classifier by $0.50 \%$ and $1.68 \%$, respectively. In other words, the addition of 22 instances to the training set of the classifier decreased its performance, which is highly unexpected. These results can be linked to the un-annotated addition of instances to the training set, which in some cases lead to a mislabeled instance being added to the training set, therefore decreasing the 
classifier's performance. Especially during the early iterations of learning, since the training set consists of such a small number of instances, the occurrence of a confidently mispredicted instance would be highly possible. Ultimately, when there is no annotation process, these predicted instances will be added to the training set as mis-labeled instances and decrease the performance of the classifier during the following iterations with their incorrect information. When we compare the final average AUPRC of this classifier against the certainty-based active learning classifier in Table 6, it can be observed that that latter increases average performance by $2.40 \%$. By considering the fact that both methods used a similar certainty-based approach and only differed in the querying of selected instances before addition to the training set, the value of querying each instance for their true class becomes evident.

By comparing our active learning strategies to a passive learning approach in Table 6, we demonstrate the value of adding highly informative instances. As with active learning, the passive learning classifier was also able to query two selected instances at each iteration for 11 learning iterations. The only difference was that the instances were selected randomly. At the final learning iteration for this approach, we have used the same labelling budget as our active learning approach, but do not get similarly good results. This underlies the importance of selecting informative points for addition to the training set. By random selection of points, despite enlarging the training set size, we are not maximizing performance. The results indicated that active learning outperforms passive learning consistently on five of the six species, where only in the cow species does passive learning perform better on most iterations (excluding the final iteration). It is unclear why passive learning is so effective for this species. Therefore, by performing this comparison we demonstrate that active learning does not simply increase classification performance by increasing training set size, rather it maximizes classification performance for a determined labeling budget. 
With the significance of using the uncertainty querying approach becoming evident, we looked towards using this strategy to demonstrate the advantages of active learning in miRNA classification. To do so, we conducted the comparison experiments demonstrated in Figure 15 and Table 7. These experiments compare active learning with sequence-based, expression-based and integrated classifiers, as well as the well-known miRNA classification tool miRDeep2. All classifiers are trained using the same number of instances as the training set size at the final iteration of the active learning classifier. The results of these comparisons demonstrate that active learning outperforms other methods in all of the data sets. It must be acknowledged that in some curves in Figure 15, the reported improvement in AUC from Table 7 is not obvious from PR curves themselves. This has to do with higher achievable precision at low recall (Curves doing much better at lower recall). For high class imbalance, it is often necessary to operate at very high precision levels, even at the expense of recall, to avoid being inundated with false positive predictions. Additionally, the precision-recall curves for the cow, chicken, and horse species in Figure 15 demonstrate sharp discontinuities at low false positive rates for some of the classifiers (especially for the miRDeep2 classifier). This can be explained by looking at Equation 6, where precision is defined as the ratio of TP to TP plus FP. Discontinuities result from the appearance of individual FP, since the ratio is highly nonlinear, especially with small sample counts.

The fact that our active learning approach greatly outperforms classifiers built on each of the views individually, demonstrates the advantage of using an integrated feature set which takes advantage of all lines of evidence. Our active learning approach is also compared against an integrated classifier similar to that presented in [68], where the classifier has the same feature set as our active learning classifier. The difference between the two methods is that no active learning is applied to the other classifier. The fact that active learning 
outperforms this method demonstrates its superior ability in selecting instances to be labelled that maximize improvement in classifier prediction accuracy. In addition, active learning's improvement compared to miRDeep2 also suggests it is a reliable and effective method for miRNA classification and can improve on the performance of the best existing miRNA classification methods.

\subsection{Conclusion}

In this chapter, we propose a novel active learning approach for the classification of miRNA. This approach takes advantage of both sequence and expression-based features to maximize pattern classification ability. Certainty and uncertainty-based active learning query methods are developed and examined to find the best approach to query instances that maximize performance increase, and it is observed that the uncertainty approach is superior by an average of $3.49 \%$ (AUPRC). The uncertainty-based active learning approach is then compared to a variety of classifiers where no active learning is applied, and shows an improved performance compared to all of them. The active learning approach is also compared to the state-of-the-art method miRDeep2 and shows an improved performance of $7.91 \%$. In general, results show that by selecting samples to be labelled that maximize improvement in classifier prediction accuracy, active learning is able to greatly improve classification performance using a very limited number of labeled instances. Therefore, evidence gathered in this chapter suggests that active learning is a suitable approach for miRNA classification. 


\section{Multi-view Co-training for miRNA Prediction}

\subsection{Introduction}

As mentioned in chapter 2, state-of-the-art miRNA prediction techniques detect pre-miRNA sequences forming miRNA-like hairpins and classify them based on the presence or absence of sequence- or expression-based features. The majority of miRNA prediction methods rely on one of the two views (i.e. sequence- and expression-based), with our recent work introducing an integrated feature set leveraging both views $[12,68]$.

Classification methods that rely on expression-based features for miRNA prediction operate on data from NGS experiments. They map the resulting collection of short reads to the genome then look for evidence of biochemical machinery integral to the miRNA biosynthesis pathway. For example, one would expect many more reads to correspond to the mature miRNA region than to the loop region (see Figure 10), since the loop region is rapidly degraded during miRNA biosynthesis. The expression profiling of pre-miRNA sequences carries a large variety of information which can be used for classification. Some studies use features such as secondary structure, total read depth of the pre-miRNA region and expression of Dicer products [63]. Other methods have also used miRNA:miRNA* duplex stability and expression level matching of NGS read data and expected Dicer processing to classify candidate pre-miRNA [60].

Sequence-based classification methods on the other hand focus on features extracted directly from the candidate pre-miRNA region of the genome, with no requirement for NGSderived expression data. Sequence-based features include local structure and sequence information of the stem-loops [55], local contiguous triplet structure composition [56] and dinucleotide features. Some methods have also considered using attributes related to 
frequencies of secondary structure triplets, amino acid string length and the size of internal loops [64] for pre-miRNA classification. Pre-miRNA hairpin features also provide valuable information on region-based structure, precursor structure and stem-loop regions of candidate miRNA genes [57].

Each of these methods create lines of evidence that have proven capable of predicting miRNA on their own. Therefore, two views exist on the problem of classifying pre-miRNA regions, where each view is independent of the other and capable of classification on its own. Being in possession of these two views, we would like to leverage them to create more powerful classifiers without actually collecting any new labelled miRNA data. In such a scenario, we hypothesize that co-training will be an effective approach for miRNA prediction, by using the two different views to iteratively create a larger labeled set. This requires building two distinct classifiers, where each of the view-based classifiers can help strengthen the performance of the other. The greatest advantage of co-training is that it will have no experimental cost, requiring no additional annotations or wet-lab validation experiments. The only expense is additional computational runtime, which pales in comparison with the cost of wet-lab experimentation.

The advantage of requiring only a minimum number of annotations when using multi-view co-training is further highlighted by considering the nature of miRNA. As previously mentioned, miRNA are very short sequences, and also highly degenerate (i.e. they do not possess a strongly conserved sequence motif) [96], which means detecting them amongst all the others sequences is a challenging task. MiRNA validation requires complex and costly wet-lab experiments such as northern blotting [22], RT-PCR [23], and microarrays [24]. In addition, considering the non-linear growth of unlabeled data available due to NGS experiments, we must consider methods that can make more use of this cheap unlabeled data instead of expensive labeled data. The development of an effective miRNA prediction 
method that does not require additional labeling procedures and makes maximum use of unlabelled data is therefore an ideal approach for this problem.

In this chapter, various learning strategies are performed in order to compare and evaluate the potential of applying co-training to miRNA prediction. Similar to the goals of the previous chapter, we aim to develop effective miRNA predictors while simulating a paucity of training data. We intend to develop effective miRNA prediction methods by making use of both expression- and sequence-based views for classification through a multi-view cotraining approach. In comparison with the active learning approaches discussed in the previous chapter, the fact that all results will come at no labeling costs is a major advantage of this proposed method. Our goal is to approach state-of-the-art prediction performance while constraining the classifiers to use using only a fraction of the available training data; achieving this goal can be viewed as a success for multi-view co-training.

\subsection{Methods}

In order to implement a successful co-training approach, two robust classifiers were created, each approaching the problem from an independent view. As previously mentioned, the views are based on two different miRNA descriptive information sets, one using sequence-based features to describe an miRNA and the other using expression-based features to do so. Following a similar methodological approach as used in the previous chapter, we first determine the size of the initial seed training set to be used by each view. Next, feature selection must be applied to identify the appropriate features to be selected for the training of each view. The choice of classifier is also important, as there is not a single algorithm which is always guaranteed to perform better than others. After the framework for the co-training algorithm is established, the number of iterations to perform 
and the number of instances to add at each iteration must also be considered. The general framework of our proposed classifier is presented in Figure 16.

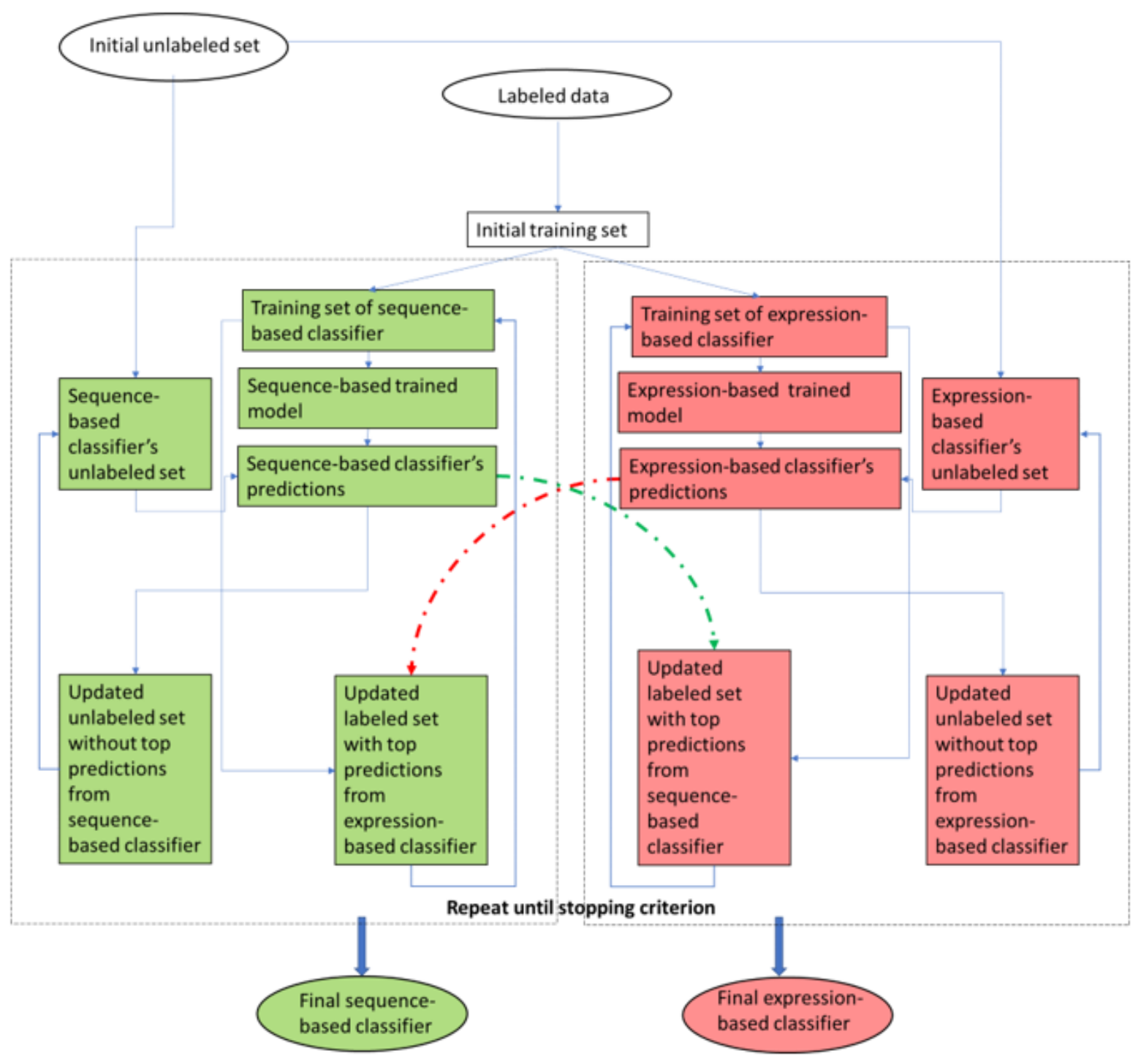

Figure 16. Co-training classifier framework repeated until stopping criterion

As can be observed from Figure 16, we used a slightly different approach compared to the standard co-training approach depicted in Figure 9. In most co-training models, a pool is created containing all the labeled data. At each iteration, the training set for each classifier 
is formed from a random subset of the total labeled pool. Also, at the end of each iteration, each classifier contributes newly labelled samples to that common pool. Our approach differs in that each classifier has its own independent labeled training data and at the completion of each iteration, each classifier contributes newly labelled data (i.e. its high confidence predictions) to the other classifier's training pool, not its own training set or to a shared pool. This method was proposed and implemented by Brefeld and Scheffer [97] and [70]. This variation of multi-view co-training ensures that the views do not converge and that they are learning from different instances, since the majority of the training sets are inclusive. Therefore, this method will further strengthen each view by directly incorporating evidence provided by another view into its training data.

Our proposed co-training algorithm is applied to 6 different species, as specified in Table 2 in Section 3.2. Co-training models have been built for each species using an identical approach to ensure the consistency of our results. Positive and negative labeled instances were collected as described in section 3.2. After removing redundant sequences and obtaining the final labeled set, an initial split of the data is applied. For each species, the data is split into $20 \%$ hold-out data, which is used for testing the performance of the classifier, and $80 \%$ which is used for training the classifier. Of the training data, only a small portion is used to create the labelled seed training set. As with active learning discussed previously, determining the size of the seed training set is an important factor, because the initial number of instances must be small enough to allow room for improvement [77].

\subsubsection{Size of Initial Seed Training Set}

We therefore determine the ideal size of the initial training set experimentally by testing on the human species data set. Random forest classifiers were used with all parameters set to the default of the SKLearn random forest library [93] and the number of trees set to 500. Random seed training sets were selected from the $80 \%$ split; the same samples were 
selected for the labelled seed training set for each view. To examine the effect of seed training set size, the number of labeled instances are incrementally increased, starting from 5 positive and 5 negative instances and adding 5 to each class until reaching 50 labeled instances in each class. This procedure is repeated 100 times using different seed samples. The results for these experiments can be viewed in Table 8 . As it can be seen, the performance of the classifier plateaus at a very small training set size, which is around 20 samples in total (10 positive and 10 negative samples). We therefore decided to use 5 positive and 5 negative labeled instances for our initial training set to allow room for improvement and to simulate the case where very few training data are available, as would be the case for a newly sequenced species.

\begin{tabular}{|c|c|c|}
\hline $\begin{array}{c}\text { \# of } \\
\text { instances in } \\
\text { training set }\end{array}$ & $\begin{array}{c}\text { Expression-based classifier's } \\
\text { average area under } \\
\text { precision-recall curve }\end{array}$ & $\begin{array}{c}\text { Sequence-based classifier's } \\
\text { average area under precision- } \\
\text { recall curve }\end{array}$ \\
\hline $\mathbf{1 0}$ & $0.5805( \pm 0.025)$ & $0.3578( \pm 0.032)$ \\
\hline $\mathbf{2 0}$ & $0.7092( \pm 0.013)$ & $0.5644( \pm 0.020)$ \\
\hline $\mathbf{3 0}$ & $0.7880( \pm 0.010)$ & $0.7643( \pm 0.013)$ \\
\hline $\mathbf{4 0}$ & $0.7895( \pm 0.007)$ & $0.7665( \pm 0.009)$ \\
\hline $\mathbf{5 0}$ & $0.7890( \pm 0.007)$ & $0.7670( \pm 0.007)$ \\
\hline $\mathbf{6 0}$ & $0.7906( \pm 0.005)$ & $0.7807( \pm 0.005)$ \\
\hline $\mathbf{7 0}$ & $0.7921( \pm 0.005)$ & $0.7801( \pm 0.004)$ \\
\hline $\mathbf{8 0}$ & $0.7956( \pm 0.005)$ & $0.7826( \pm 0.004)$ \\
\hline $\mathbf{9 0}$ & $0.7977( \pm 0.004)$ & $0.7817( \pm 0.004)$ \\
\hline $\mathbf{1 0 0}$ & $0.8119( \pm 0.004)$ & $0.7879( \pm 0.004)$ \\
\hline
\end{tabular}

Table 8. Area under precision-recall curve for sequence- and expression-based classifiers using different training set numbers

\subsubsection{Feature Selection}

Feature selection is then applied to identify the appropriate features to be selected for the training of each view. This is done to reduce the number of features by omitting the redundant or less informative ones. As mentioned in Section 3.3, a total of 215 sequencedbased and 8 expression-based features are initially derived from the data sets. In order to 
obtain a single feature set with a good generalization across all species, the entire $80 \%$ split data from all six species is used. The correlation-based feature subset selection method in the Weka package [94] is applied to all the data from the six species, once for expressionbased features and once for sequence-based features. This correlation-based feature subset selection algorithm selects the most informative features based on the lack of intercorrelation between the features and the ability of a feature to predict the class. Using this method to obtain the optimal feature set for each view resulted in 32 features for the sequence-based classifier and 8 features for the expression-based classifiers. These features are used for the training of all co-training experiments in this thesis.

\subsubsection{Classifier Model Selection}

Classifier selection is also done experimentally. An ideal classifier should result in a balance between sensitivity and specificity. As mentioned in Section 2.2, most previous works in the field of miRNA prediction have used random forest or SVM as their classifier. Zou et al. found random forest to have the best performance after testing different models [65]. Other studies have also suggested that random forest yields the best balance between sensitivity and specificity and outperforms other classifier types for miRNA prediction [10, 64], while yet other studies have suggested the use of SVM for this classification problem $[55,58]$. We decided to test both recommended methods to see which one yields the best result. Similar to what we did with the selection of the training set size, we tested SVM and random forest classifiers on the human data set.

Two co-training frameworks were developed, one using SVM classifiers and one using decision tree classifiers. Each used 10 labeled samples as the initial training set (5 positive and 5 negative) with the optimal feature sets obtained previously for sequence and expression-based views. The SVM classifier is built using a linear kernel with all other parameters set to the default of the SKLearn SVM library [93]. The random forest classifier 
was also built using the default parameters of the SKLearn random forest library [93], with the number of trees set to 500 . The $80 \%$ split of the data was used for training and $20 \%$ was used for performance evaluation. The same initial training set is used for both SVM and random forest classifiers. At each iteration, the classifiers were trained based on the training data, and are then applied to the remaining $80 \%$ of the data to choose the most confident predictions for addition to the other view's labeled training set. The performance of the iteration is then tested on the $20 \%$ hold-out set, where all labels have been held back and are only used for performance estimation. After applying the trained model to the unlabeled set at each iteration, two of the most confident predictions, one for the negative and one for the positive class, are added to the training set of the other classifier. Six iterations were performed in this manner to compare the two classifiers and choose the best performing one to use for all experiments. The results of these experiments can be viewed in Table 9. As can be observed, the random forest classifier performs better than the SVM classifier in both views and consistently across iterations. Based on these preliminary findings, we opted to use random forest as our classifier for all subsequent co-training experiments.

\begin{tabular}{|c|c|c|c|c|}
\hline Iteration & $\begin{array}{c}\text { SVM expression- } \\
\text { based } \\
\text { classifier's } \\
\text { AUPRC }\end{array}$ & $\begin{array}{c}\text { RF } \\
\text { expression- } \\
\text { based } \\
\text { classifier's } \\
\text { AUPRC }\end{array}$ & $\begin{array}{c}\text { SVM } \\
\text { sequence- } \\
\text { based } \\
\text { classifier's } \\
\text { AUPRC }\end{array}$ & $\begin{array}{c}\text { RF sequence- } \\
\text { based } \\
\text { classifier's } \\
\text { AUPRC }\end{array}$ \\
\hline $\mathbf{1}$ & $0.5731( \pm 0.03)$ & $0.5965( \pm 0.03)$ & $0.3386( \pm 0.03)$ & $0.3436( \pm 0.03)$ \\
\hline $\mathbf{2}$ & $0.5927( \pm 0.02)$ & $0.6813( \pm 0.02)$ & $0.4386( \pm 0.03)$ & $0.4477( \pm 0.02)$ \\
\hline $\mathbf{3}$ & $0.6456( \pm 0.02)$ & $0.7052( \pm 0.02)$ & $0.5190( \pm 0.03)$ & $0.5681( \pm 0.02)$ \\
\hline $\mathbf{4}$ & $0.6558( \pm 0.02)$ & $0.7209( \pm 0.02)$ & $0.6291( \pm 0.02)$ & $0.6784( \pm 0.02)$ \\
\hline $\mathbf{5}$ & $0.6773( \pm 0.01)$ & $0.7523( \pm 0.01)$ & $0.6569( \pm 0.02)$ & $0.7355( \pm 0.01)$ \\
\hline $\mathbf{6}$ & $0.6862( \pm 0.01)$ & $0.7483( \pm 0.01)$ & $0.6909( \pm 0.02)$ & $0.7345( \pm 0.01)$ \\
\hline
\end{tabular}

Table 9. Performance of sequence and expression-based views for RF and SVM over 6 iterations of co-training on the human dataset. Means \pm standard deviations are shown, representing 100 repetitions of each experiment. 


\subsubsection{Co-Training Stopping Criterion}

Our next task was to determine the optimal number of iterations to perform for each experiment and the number of instances to add at each iteration. Adding too many points at each iteration may mean adding samples with a lower confidence, which can result in misclassified instances being added to the training set and a decrease in performance. Too many iterations will greatly lengthen the time and computational cost of the algorithm. To avoid the addition of low confidence samples to the training set, we decided to add two samples: the sample predicted to be positive and negative with the highest confidence level. Therefore, at every iteration, two instances are added to the training set of each view of the model. By doing so we hope to maintain a relatively high confidence percentage for all added points throughout the iterations.

In order to determine the number of learning iterations to perform, as with active learning, we here decided to follow the general semi-supervised learning stopping criterion approach suggested by Lewis and Gale [7]. In this strategy, labeling will stop once it is determined that the classifier has reached its maximum performance effectiveness. The effectiveness of the classifier is evaluated by testing its performance at each iteration on a labeled data set where the labels are preserved and only used for performance evaluation. Understandably, the data set used for performance evaluation must be independent of the training set and the unlabeled prediction set. We use the $20 \%$ hold-out data for performance estimation and construct the learning curves of each classifier to see when the maximum performance is reached. Once we reach maximum effectiveness, meaning the learning curves plateau and we observe that new iterations do not increase performance, we will stop the learning and annotation process. By applying this procedure to our data sets, it was found that in most cases maximum performance effectiveness is reached between the $10^{\text {th }}$ and $11^{\text {th }}$ iterations. Figure 17 demonstrates this fact for the human data set as an example, where it is 
observed that performance remains relatively constant towards the final iterations. Therefore, we set a static threshold of 11 learning iterations for all experiments.
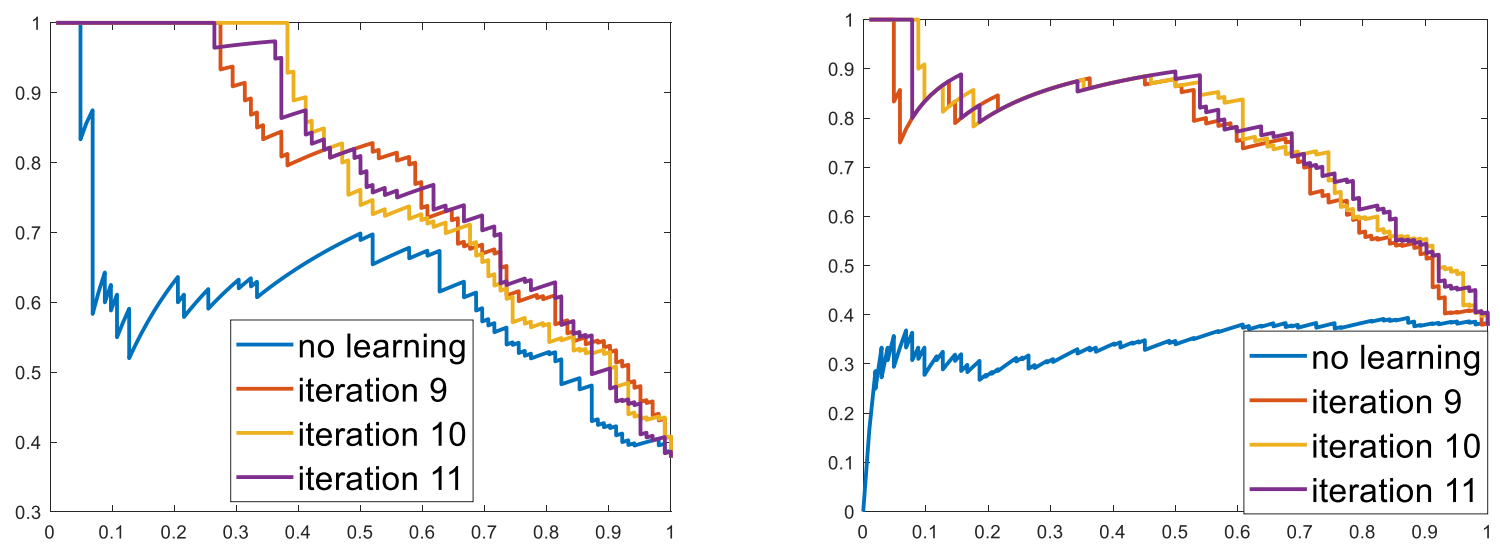

Figure 17. Average precision-recall curve for human data set at specific iterations for expression (left figure) and sequence-based (right figure) classifiers. The y-axis represents precision while the $x$-axis is recall.

\subsubsection{Summary of Co-Training Approach}

As a summary, our main classifier for each view is a random forest classifier with 500 trees and the default parameters of the SKLearn random forest library [93], and a starting training set of 10 labeled samples. The initial model for each view will be built on this training set using the view's related feature set. After the model is built, it is applied to the $80 \%$ unlabeled set, where the single most confident positive and negative predictions will be selected by each view. These 2 instances will be removed from the unlabeled set of their own view and added to the training set of the opposite view. The classifier for each view is re-trained using the new training set, and this procedure is continued for several iterations. After each iteration, the learning curves of each view is plotted, where AUPR is used as the summary performance metric. The trends of these learning curves are studied, and the learning process will stop once it is observed that maximum effectiveness is reached and performing extra iterations does not result in increase in performance. 


\subsubsection{Benchmark Algorithms}

In the next section, we will observe the results of our proposed method of co-training and will compare these results with three benchmark algorithms: a passive approach where points are added randomly, the no learning case, and self-training. These algorithms are described below.

The passive co-training strategy applied is built using the same setup used for the cotraining classifier, with the same random forest classifier, feature sets and training and test sets. In this passive strategy, in contrast to the actual co-training strategy where only the most confidently predicted instances are added to the training set, here instances are selected and added to the training set randomly. In this approach, at each iteration the two random forest classifiers built using the two view-based feature sets and the training data are applied to the $80 \%$ unlabeled set. For each view, two randomly selected instances will be removed from the unlabeled set and added to the training set of the other view. Rather than using the actual class, as done in active learning, here the predicted class labels are used. The selected instances were not selected completely at random; rather, they were selected randomly from a group of predictions expected to be either in the positive or negative class. To explain further, when the model is applied to the unlabeled set, each of the unlabeled instances receives a prediction score. This score indicates the probability of that instance being in the positive or negative class, with a range of 0 to $100 \%$ probability for each class. In the passive approach, we randomly select one instance that is more than $50 \%$ predicted to be a positive, and one that is more than $50 \%$ predicted to be a negative. In this way, we are hoping to add one positive and one negative at each iteration to keep the training set of the passive approach in balance with that of co-training. This process is repeated for the same number of iterations as the active learning strategy to compare the performances on the final training set using the same number of training instances. By 
comparing our co-training classifier's performance to that of this passive classifier, we can determine the significance of adding the most confidently predicted points in performance.

Self-training is another benchmark algorithm that we use to compare against our cotraining approach. In self-training, each view is strengthened by its own predictions, meaning that each view will be used for classification independently [98-100]. In this approach, we create two classifiers, one for each of the sequence- and expression-based views. Each classifier is built using the same random forests, view-specific feature sets, and the same training, testing and unlabeled data sets previously described. Each classifier is built using the training set and one of the views, and at each iteration, the trained model is applied to the unlabeled set. The single most confident positive and negative predictions are then removed from the unlabeled set and added to the training set of the view itself. This process is continued for the same number of iterations as co-training to compare the results. By making this comparison, the value of using different views to strengthen each other can be observed. It is hypothesized that co-training will outperform self-training since using one's own predictions to reinforce one's beliefs is expected to lead to overspecialization and reinforcement of a classifier's errors. This is avoided in co-training since each classifier augments the training set of the other view, rather than its own.

Lastly, the final iterations of co-training, passive learning and self-training are compared against a classifier without any learning being applied (using the initial seed of 10 samples), referred to as the "no learning" classifier. The final performance of all methods are plotted on a precision-recall curve, and the performance of the expression and sequence-based views of co-training are reported at each iteration. The classifier's performance at each iteration is evaluated by calculating the area under the precision-recall and ROC curves. Prevalence-corrected precision is calculated instead of precision in all experiments to 
compensate the fact that the experiment sets may not accurately reflect the true class imbalance for each species.

\subsection{Results}

Our proposed co-training classifier is applied to the six data sets described in section 3.2. The results of each of the final expression- and sequence-based classifiers after applying cotraining to the data sets are obtained and reported. In addition to applying co-training to these data sets, three benchmark strategies are also applied to better comprehend the performance of our approach.

For all the experiments, a $20-80 \%$ data split is first applied, where the $20 \%$ split of the data

is held back for performance evaluation. From the remaining $80 \%$ of the data, 5 positive and 5 negative instances are used to construct the initial training set of the classifiers, and the rest are treated as unlabeled data which are used for prediction and addition to the training set. After each iteration, the two selected instances are removed from the unlabeled set and added to the training set. This distribution of data is randomly repeated 100 times, resulting in different seed training sets, test sets and unlabeled sets. Therefore, each learning strategy is evaluated 100 times using different initial seed training sets, and all plots show the mean of 100 repetitions.

\subsubsection{Establishing the Effectiveness of Co-Training for miRNA Prediction}

We have included a table reporting performance at each iteration for the human species to demonstrate the performance of our co-training method. For validation purposes, we also applied the same method to the other five species and have included the results tables in Appendix $A$ of this thesis. The results show the performance of the sequence- and expression-based classifiers developed using co-training using the area under precisionrecall curve metric at each iteration. A total of 11 iterations are performed, resulting in a 
total of 22 instances being added to the initial training set. Therefore, the results on the last iteration show the performance of a classifier trained using only 10 labeled and 22 pseudolabeled instances. Table 10 shows the results obtained at each iteration by applying cotraining to the sequence and expression-based views on the human data set.

\begin{tabular}{|c|c|c|c|c|}
\hline $\begin{array}{c}\text { Co- } \\
\text { training } \\
\text { iteration }\end{array}$ & $\begin{array}{c}\text { expression-based } \\
\text { average AUPRC }\end{array}$ & $\begin{array}{c}\text { sequence-based } \\
\text { average AUPRC }\end{array}$ & $\begin{array}{c}\text { expression- } \\
\text { based average } \\
\text { AUROC }\end{array}$ & $\begin{array}{c}\text { sequence- } \\
\text { based average } \\
\text { AUROC }\end{array}$ \\
\hline- & $0.596( \pm 0.03)$ & $0.344( \pm 0.03)$ & $0.735( \pm 0.02)$ & $0.465( \pm 0.03)$ \\
\hline $\mathbf{1}$ & $0.681( \pm 0.02)$ & $0.448( \pm 0.02)$ & $0.748( \pm 0.02)$ & $0.581( \pm 0.03)$ \\
\hline $\mathbf{2}$ & $0.705( \pm 0.02)$ & $0.568( \pm 0.02)$ & $0.793( \pm 0.02)$ & $0.711( \pm 0.03)$ \\
\hline $\mathbf{3}$ & $0.721( \pm 0.02)$ & $0.678( \pm 0.02)$ & $0.775( \pm 0.02)$ & $0.789( \pm 0.02)$ \\
\hline $\mathbf{4}$ & $0.752( \pm 0.01)$ & $0.735( \pm 0.01)$ & $0.813( \pm 0.01)$ & $0.826( \pm 0.01)$ \\
\hline $\mathbf{5}$ & $0.748( \pm 0.01)$ & $0.734( \pm 0.01)$ & $0.830( \pm 0.01)$ & $0.838( \pm 0.01)$ \\
\hline $\mathbf{6}$ & $0.781( \pm 0.01)$ & $0.739( \pm 0.01)$ & $0.833( \pm 0.01)$ & $0.826( \pm 0.01)$ \\
\hline $\mathbf{7}$ & $0.771( \pm 0.01)$ & $0.747( \pm 0.01)$ & $0.830( \pm 0.01)$ & $0.817( \pm 0.01)$ \\
\hline $\mathbf{8}$ & $0.791( \pm 0.01)$ & $0.744( \pm 0.01)$ & $0.833( \pm 0.01)$ & $0.819( \pm 0.01)$ \\
\hline $\mathbf{9}$ & $0.772( \pm 0.01)$ & $0.738( \pm 0.01)$ & $0.828( \pm 0.01)$ & $0.832( \pm 0.01)$ \\
\hline $\mathbf{1 0}$ & $0.773( \pm 0.01)$ & $0.761( \pm 0.01)$ & $0.824( \pm 0.01)$ & $0.840( \pm 0.01)$ \\
\hline $\mathbf{1 1}$ & $0.779( \pm 0.01)$ & $0.761( \pm 0.01)$ & $0.840( \pm 0.01)$ & $0.841( \pm 0.01)$ \\
\hline
\end{tabular}

Table 10. Co-training performance results for human data set. Means \pm standard deviations are shown, representing 100 repetitions of each experiment.

Figure 18 is constructed using Table 10 for the human data set and the corresponding tables provided in Appendix A for the other five species. Figure 18 demonstrates the mean performance of the sequence and expression-based classifiers at each iteration of cotraining for all data sets for 100 experiments with randomly selected seeds. In the human data set, the model is tested on 102 positive and 168 negative instances at each iteration. After 11 iterations of learning, the expression-based classifier's average AUPRC increases by $30.6 \%$ and it's AUROC increases by $14.3 \%$. After the same number of iterations, the sequence-based classifier more than doubles in average AUPRC, undergoing a $121 \%$ increase, and is almost doubled in average AUROC where it is increased by $81 \%$. The sequence and expression-based classifiers trained using co-training on the mouse data set are tested on 73 positive and 169 negative instances at each iteration. After 11 learning 
iterations, the expression-based classifier experiences a $33.7 \%$ increased average AUPRC and $7.60 \%$ increased average AUROC. The sequence-based classifier's average AUPRC is increased by $11.0 \%$ and AUROC is increased by $2.78 \%$.

The fruit-fly test set used for performance evaluation at each iteration consists of 22 positive and 19 negative instances. The expression-based classifier's average AUPRC increases by $11.3 \%$, where it's AUROC increases by $16.5 \%$ after 11 iterations. The sequence-based classifier experiences a $6.60 \%$ improvement in AUPRC and $5.30 \%$ in average AUROC. The results of the cow data set, tested on 66 positive and 130 negative instances, demonstrate a $12.5 \%$ improvement in the expression-based classifier's average AUPRC and a $4.90 \%$ improvement in its average AUROC after 11 iterations. The sequencebased classifier uses the same number of iterations to more than double its average AUPRC, resulting in an increase of $126 \%$, and experiencing a $59.8 \%$ average AUROC increase. The chicken test set consists of 39 positive and 21 negative instances. The application of 11 iterations of co-training resulted in the expression-based classifier's average AUPRC and AUROC increasing by $4.12 \%$ and $4.44 \%$, respectively. Co-training also increases the sequence-based classifier's AUPRC by $3.80 \%$ and its AUROC by $6.26 \%$, once applied for 11 iterations. When applied to the horse data set, with a test set consisting of 73 positive and 46 negative instances, the expression-based classifier's average AUPRC and AUROC is enhanced by $4.05 \%$ and $2.65 \%$ after 11 iterations, respectively. The sequence-based classifier undergoes an increase in average AUPRC by $9.77 \%$ and $12.9 \%$ in average AUROC. 

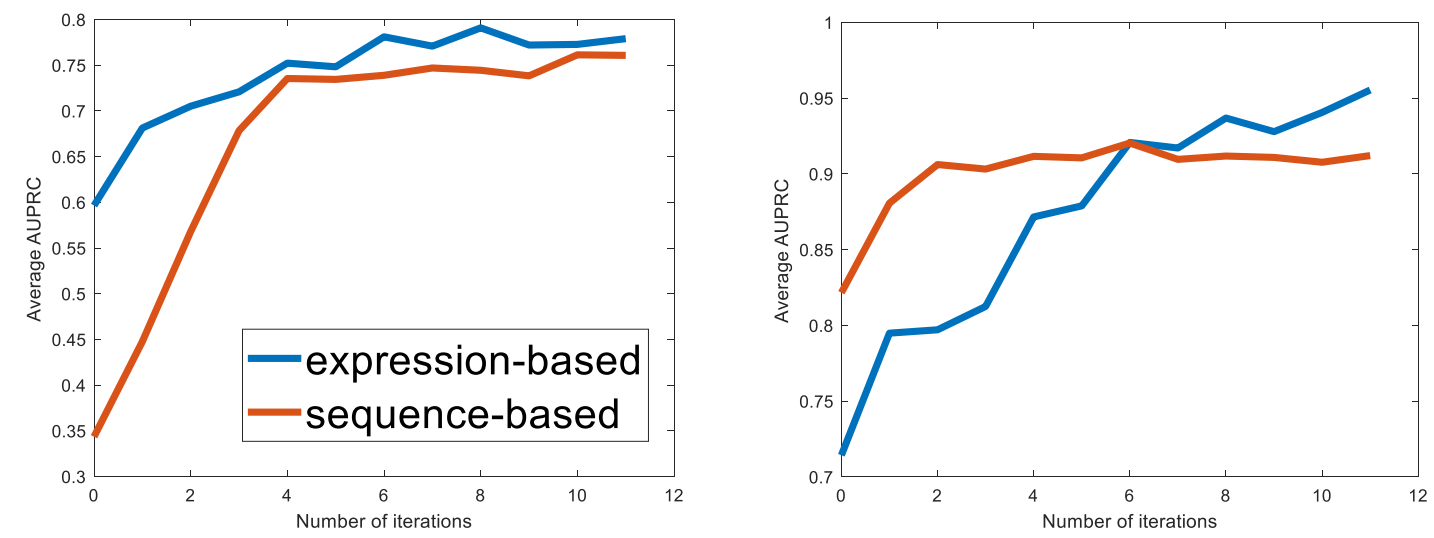

dme
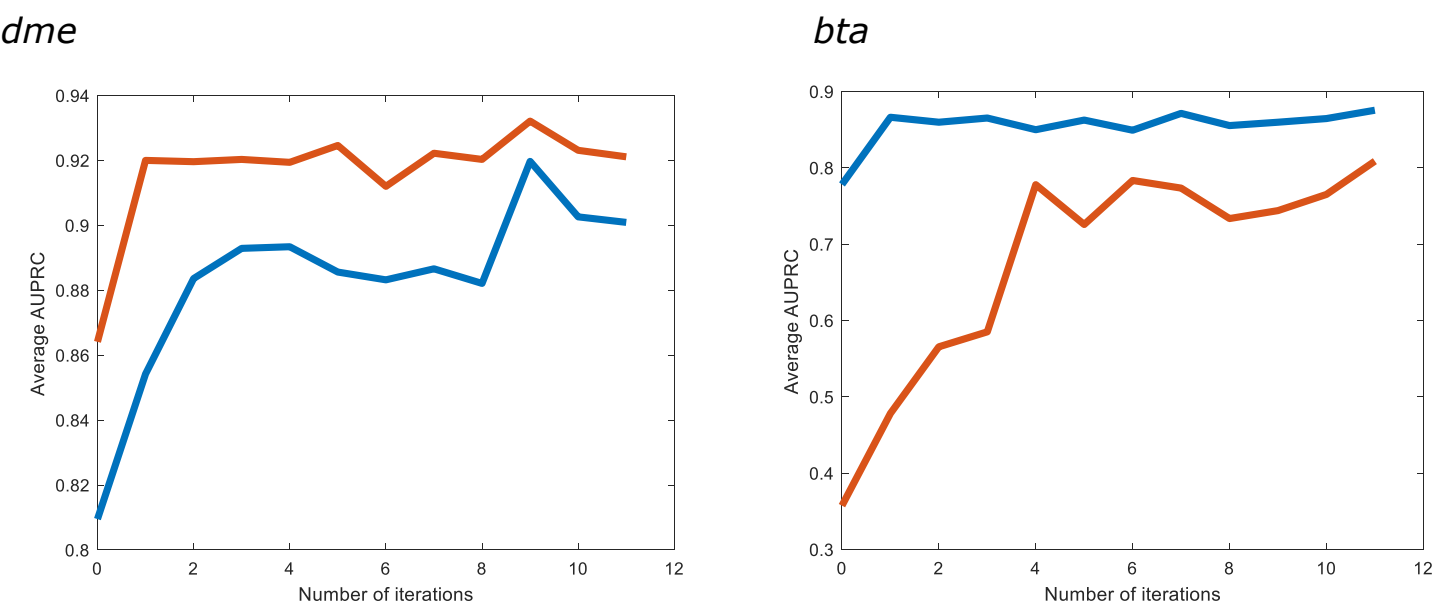

gga

$$
\text { eca }
$$
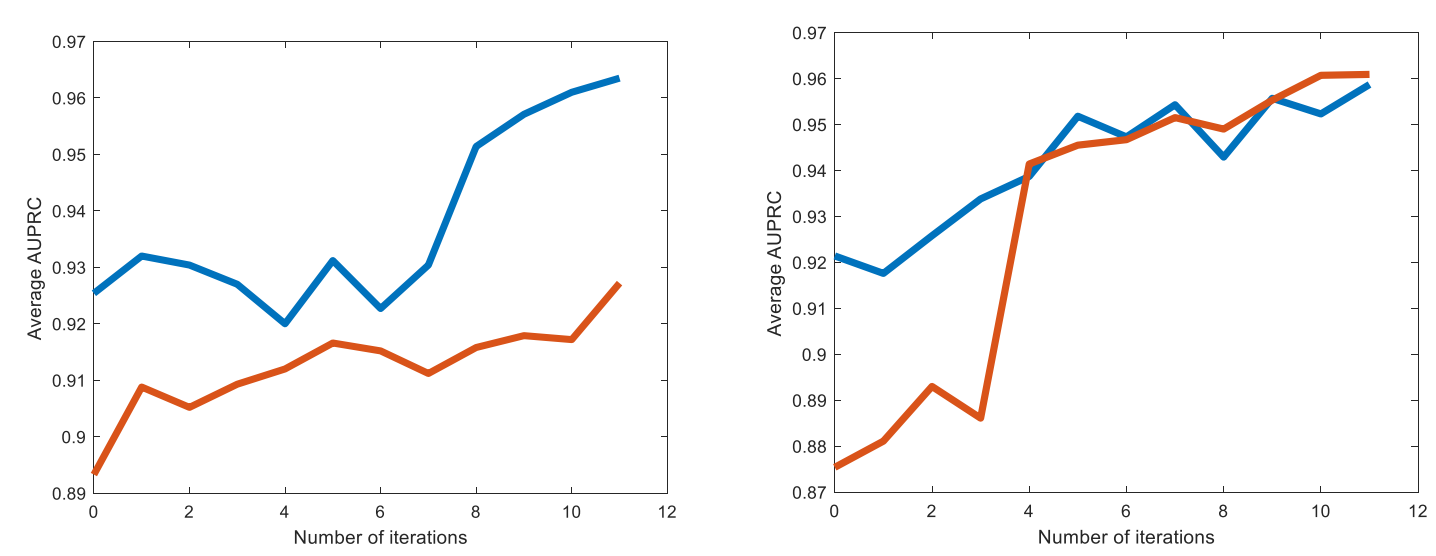

Figure 18. Performance of the sequence and expression-based classifiers at each iteration of co-training for all data sets 


\subsubsection{Comparing Co-Training with Benchmark Methods}

Table 11 compares the final result of classification using co-training, single view (selftraining), passive learning, and no learning for both views as a mean of 100 random-seed repetitions. When looking at the final expression-based classifiers resulting from each of the methods, it can be observed that, in all six species, co-training outperforms the other methods. The average performance increase across all species obtained using co-training is $15.81 \%$, compared to $11.90 \%$ for self-training, and $4.84 \%$ for passive learning. Selftraining was the second-best method, showing consistent improvement in performance compared to the no learning classifier. Apart from being the weakest method amongst the three, passive learning also performed worse than the original no learning classifier on the mouse and chicken data sets. By comparing the sequence-based classifiers yielded by each method, once again it is observed that the co-training classifier offers the best results. Cotraining results in an average performance increase of $46.47 \%$ across all species, where self-training and passive learning show $39.53 \%$ and $29.43 \%$ improvements, respectively. In the sequence-based view, all three methods consistently show improved performances compared to the no learning classifier, however the performance increases vary greatly. The maximum performance increase using co-training is $126.3 \%$ (bta), compared to $109.66 \%$ for self-training (hsa) and $83.1 \%$ for passive learning (hsa). By viewing the performance of each method both on the species-specific data sets and on average in Table 11, it becomes apparent that using the co-training approach results in a better final classifier. The standard deviation for these experiments is reported in Table A12 in Appendix A. 


\begin{tabular}{|c|c|c|c|c|c|c|c|c|}
\hline \multirow{2}{*}{$\begin{array}{l}\text { Data } \\
\text { set }\end{array}$} & \multicolumn{4}{|c|}{ Expression-based classifier's average AUPRC } & \multicolumn{4}{|c|}{ Sequence-based classifier's average AUPRC } \\
\hline & $\begin{array}{l}\text { No } \\
\text { learning }\end{array}$ & $\begin{array}{l}\text { Passive } \\
\text { learning }\end{array}$ & Self-training & Co-training & $\begin{array}{l}\text { No } \\
\text { learning }\end{array}$ & $\begin{array}{l}\text { Passive } \\
\text { learning }\end{array}$ & Self-training & Co-training \\
\hline hsa & 0.597 & $0.672(+12.7 \%)$ & $0.770(+29.0 \%)$ & $0.779(+30.6 \%)$ & 0.344 & $0.616(+79.3 \%)$ & $0.720(+109.66 \%)$ & $0.761(+121.4 \%)$ \\
\hline$m m u$ & 0.714 & $0.708(-0.83 \%)$ & $0.883(+23.7 \%)$ & $0.955(+33.7 \%)$ & 0.822 & $0.887(+7.91 \%)$ & $0.895(+8.87 \%)$ & $0.912(+11.0 \%)$ \\
\hline$d m e$ & 0.810 & $0.883(+9.10 \%)$ & $0.886(+9.50 \%)$ & $0.901(+11.3 \%)$ & 0.864 & $0.909(+5.16 \%)$ & $0.912(+5.51 \%)$ & $0.921(+6.6 \%)$ \\
\hline bta & 0.778 & $0.827(+6.23 \%)$ & $0.815(+4.70 \%)$ & $0.865(+11.1 \%)$ & 0.357 & $0.654(+83.1 \%)$ & $0.732(+104.7 \%)$ & $0.809(+126.3 \%)$ \\
\hline$g g a$ & 0.925 & $0.923(-0.22 \%)$ & $0.946(+2.26 \%)$ & $0.964(+4.12 \%)$ & 0.893 & $0.894(+0.08 \%)$ & $0.911(+2.03 \%)$ & $0.927(+3.79 \%)$ \\
\hline$e c a$ & 0.921 & $0.941(+2.11 \%)$ & $0.942(+2.25 \%)$ & $0.958(+4.02 \%)$ & 0.875 & $0.884(+0.99 \%)$ & $0.932(+6.43 \%)$ & $0.961(+9.74 \%)$ \\
\hline $\begin{array}{l}\text { Avg } \\
\text { incr. }\end{array}$ & - & $+4.84 \%$ & $+11.90 \%$ & $+15.81 \%$ & - & $+29.43 \%$ & $+39.53 \%$ & $+46.47 \%$ \\
\hline
\end{tabular}

Table 11. Final classification results using co-training, single-view (self-training), passive learning and no learning for expression and sequence-based views

Figure 19 compares the final learning curves (mean of 100 random-seed experiments) for classification using co-training, single-view (self-training), passive learning, and no learning for both views on the human, mouse and fruit-fly data sets. By constructing these learning curves and comparing our multi-view co-training approach against a single-view and passive approach, we can better comprehend the value of using both views and adding the most confidently predicted instances to the training set. For the human data set, in the expression-based view, the learning curve for co-training shows an increase of $15.9 \%$ over the passive and $1.22 \%$ over the single-view classifier. In the sequence-based view, cotraining increases performance by $23.5 \%$ and $5.61 \%$ compared to passive learning and selflearning, respectively. Co-training shows an increase of $34.9 \%$ and $8.15 \%$ over passive learning and self-training for the expression-based view applied to the mouse data set. The sequence-based view increases performance over these classifiers by $2.82 \%$ and $1.90 \%$, respectively. The expression-based classifier trained using co-training and applied to the fruit-fly data set outperforms passive learning and self-training by $2.03 \%$ and $1.70 \%$, while the sequence-based classifier outperforms the named methods by $1.32 \%$ and $0.99 \%$, respectively. 
Figure 19 is comparable to Figure 20 but illustrates results for the cow, chicken, and horse data sets. In the cow data set, the co-training expression-based classifier outperforms passive learning and self-training by $4.59 \%$ and $6.13 \%$, while the sequence-based classifier outperforms the named methods by $23.7 \%$ and $10.5 \%$, respectively. When applied to the chicken data set, the co-training expression-based classifier bests the performance of passive learning and self-training by $4.44 \%$ and $1.90 \%$, while the sequence-based classifier outperforms them by $3.70 \%$ and $1.76 \%$, respectively. In the horse data set, the co-training expression-based classifier beats passive learning and self-training by $1.81 \%$ and $1.70 \%$, while the sequence-based classifier does so by $8.71 \%$ and $3.11 \%$, respectively.

By referring to the results in Table 11 and Figures 19-20 and comparing the average AUPRC and learning curves of the different classifiers, it is observed that the performance increase in expression and sequence-based views using co-training is superior than the other two methods both on average and for each of the individual species. These results were obtained after 11 iterations of learning using the three different strategies, having the same experimental setup and initial training and test sets, and being repeated 100 times. By demonstrating substantially better results than the passive learning and self-training classifiers, it can be concluded that miRNA classification using co-training is the superior method. 
hsa: Expression

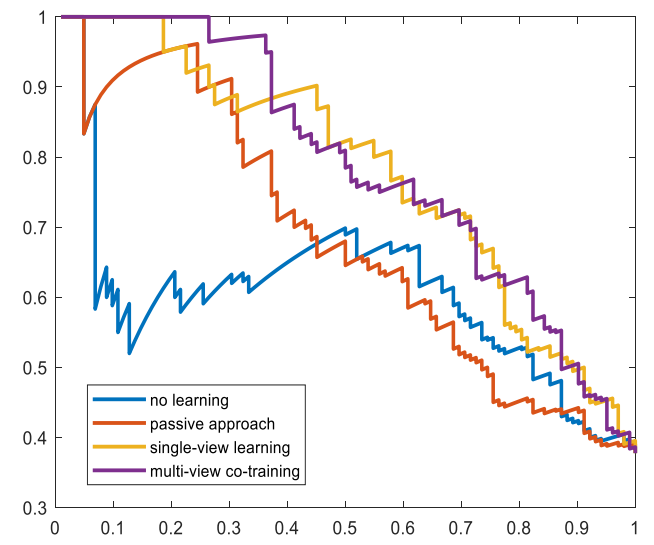

mmu: Expression

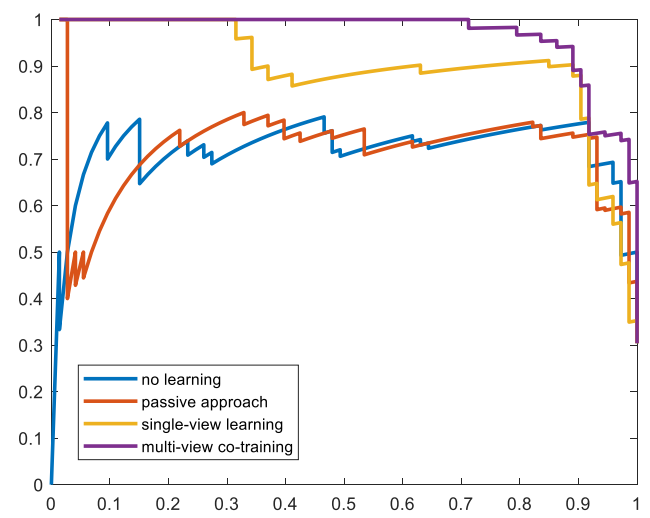

dme: Expression

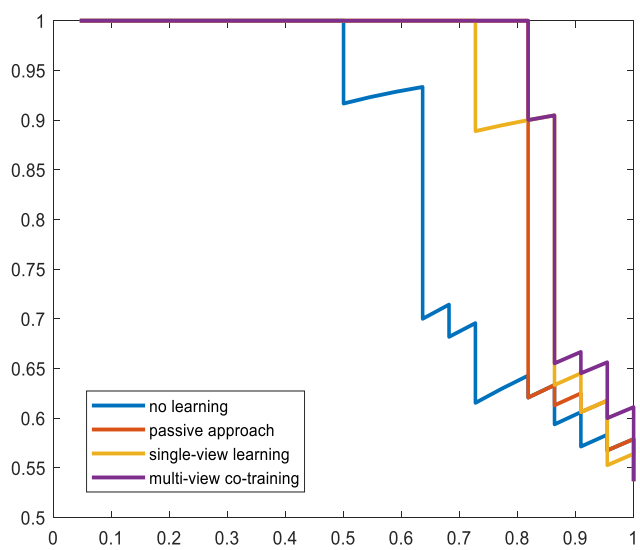

Sequence

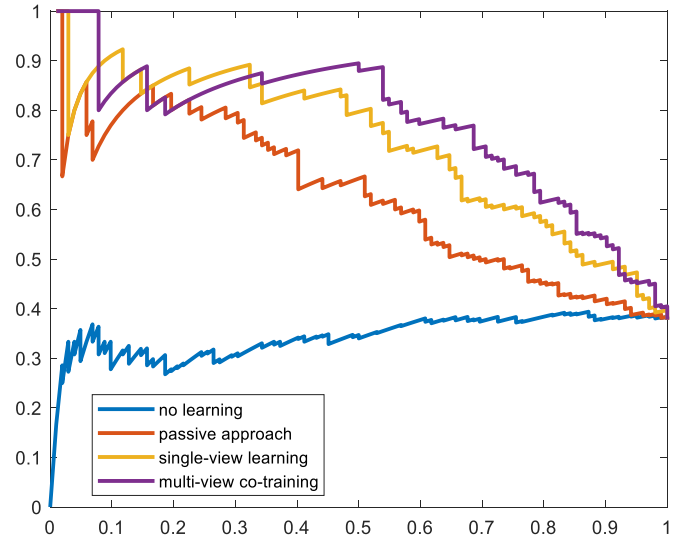

Sequence

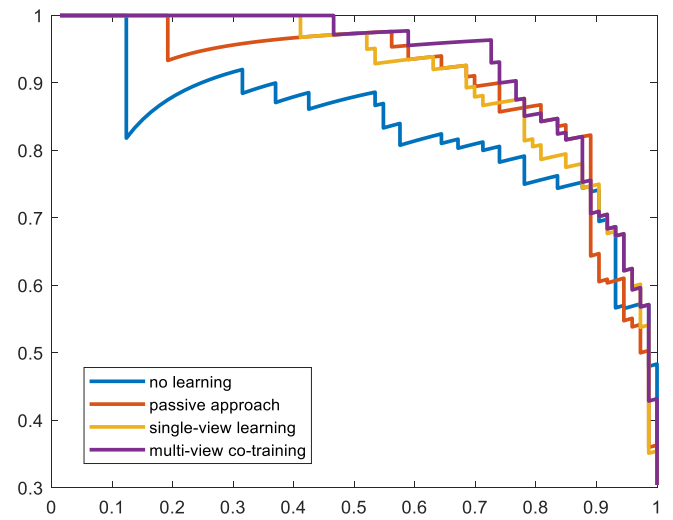

Sequence

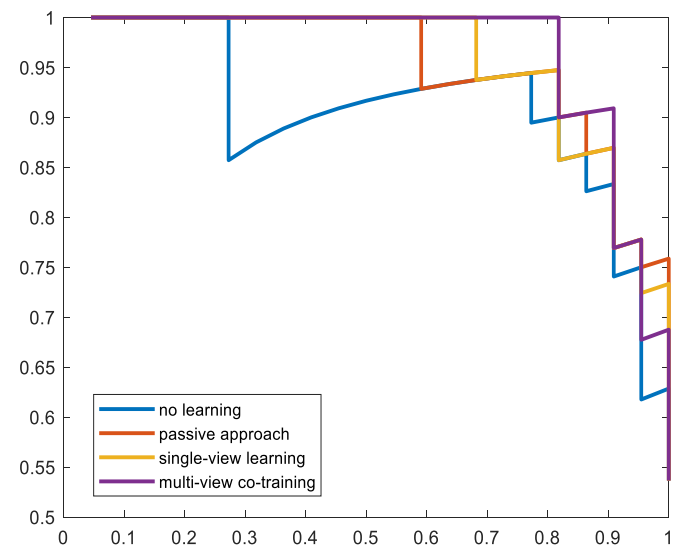

Figure 19. Final learning curves for classification using co-training, single-view (selftraining), passive learning and no learning for expression and sequence-based views on the human, mouse and fruit-fly data sets. In all plots, the y-axis represents precision while the $x$-axis is recall. Optimal performance is in the top-right corner of the plot. 
bta: Expression

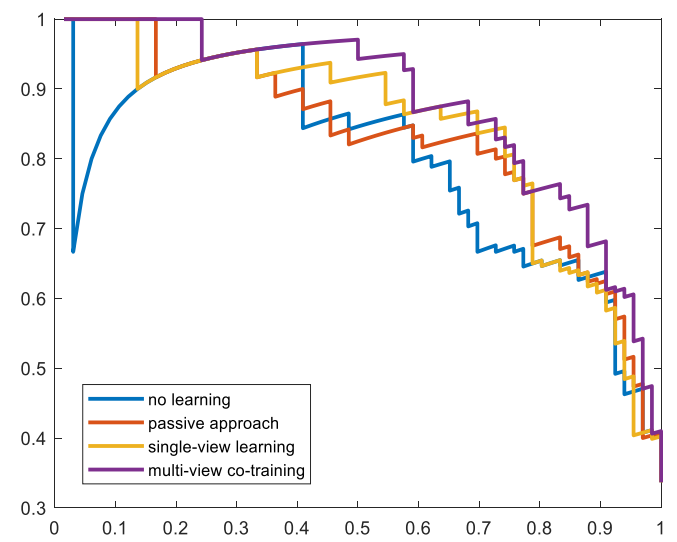

gga: Expression

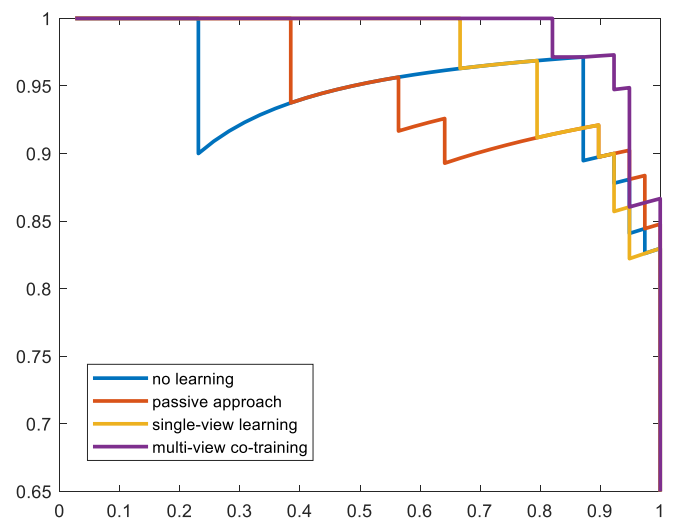

eca: Expression

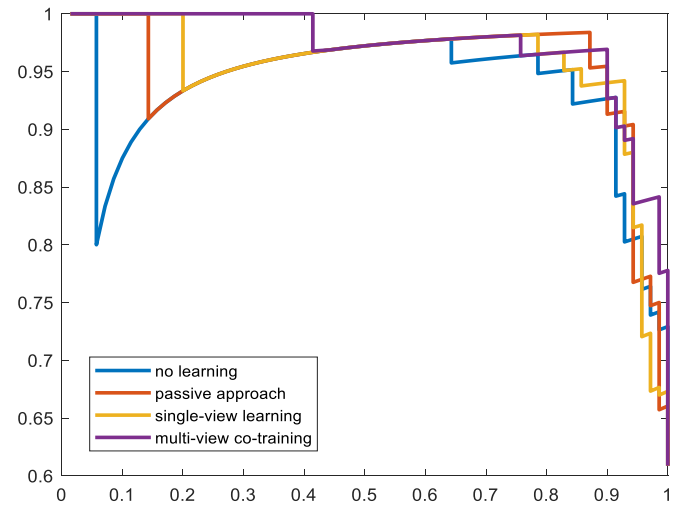

Sequence

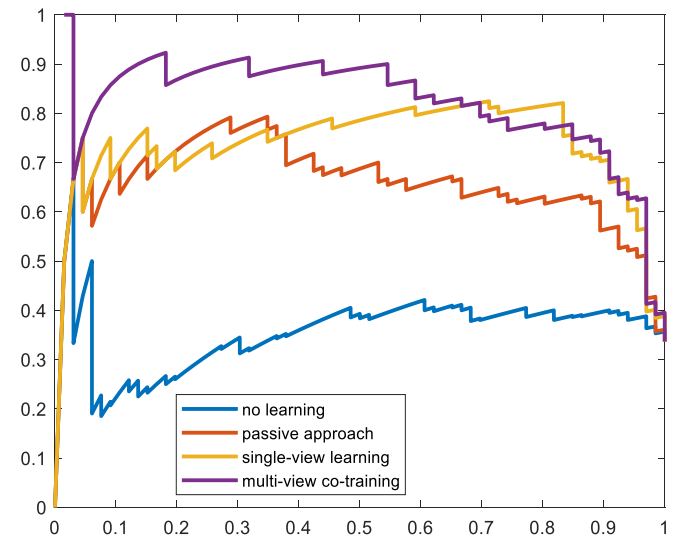

Sequence

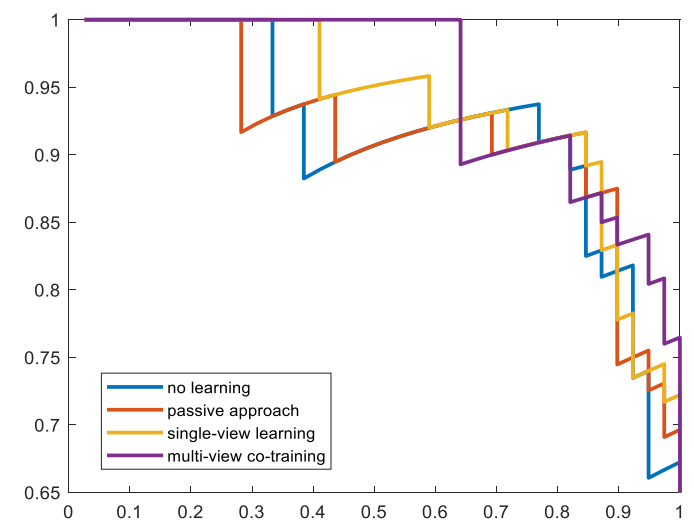

Sequence

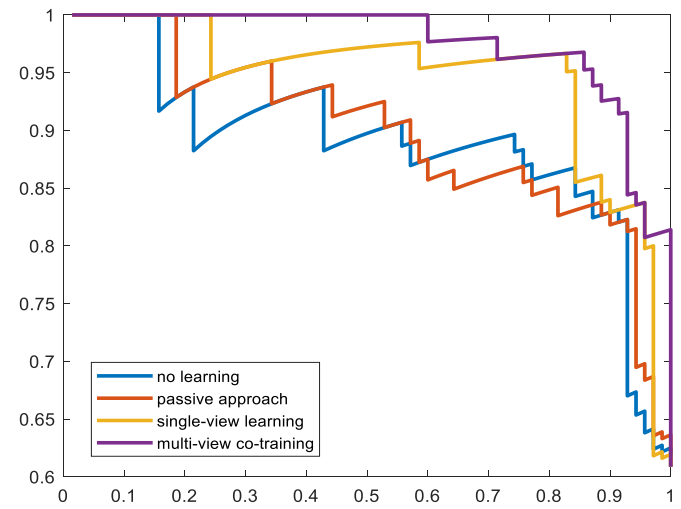

Figure 20. Final learning curves for classification using co-training, single-view (selftraining), passive learning and no learning for expression and sequence-based views on the cow, chicken and horse data sets. In all plots, the $y$-axis represents precision while the $x-$ axis is recall. Optimal performance is in the top-right corner of the plot. 


\subsubsection{Combining Views for Consensus Decision}

So far, we have evaluated the performance of each of the individual views developed using multi-view co-training. Co-training is frequently used as a means to create a larger labeled data set, rather than to perform classification. However, if we were to use co-training itself as a classification tool, it would be better to combine both developed views at the end of their learning to produce a more robust classifier. Several ways can be though of to combine the multiple views in co-training to arrive at a consensus decision. For the "classic" co-training approach [44], there is a single pool of quasi-labelled training data. Therefore, a final integrated classifier can be trained from that common pool, leveraging features from both views. In the cases where there are three or more views available for the problem, voting amongst the views for the class of the instance would be another reasonable approach. In our work, we create a combined co-training classifier considering the prediction confidence of both views for each instance. We do so using the probability score produced by our algorithm for each instance (refer to Section 3.4). When we obtain each views' probability score for an instance, we compare the two scores produced by each view, and give the preference to the view with the higher confidence in prediction. For example, if an instance is predicted by the sequence-based view to have a $70 \%$ probability of being a miRNA, while the expression-based classifier predicts it to have a $60 \%$ probability of not being a miRNA, the combined co-training classifier will predict the instance to be a miRNA.

We apply our proposed combined co-training algorithm to the data we had previously gathered for the individual views. The performance of the expression and sequence-based classifiers and the combined co-training approaches are compared in Figure 21 in terms of the average area under precision recall curves. These results demonstrate the mean of 100 experiments with random seeds, as previously mentioned. As expected, the combined co- 
training shows enhanced performance compared to each of the individual views on all data sets.

hsa

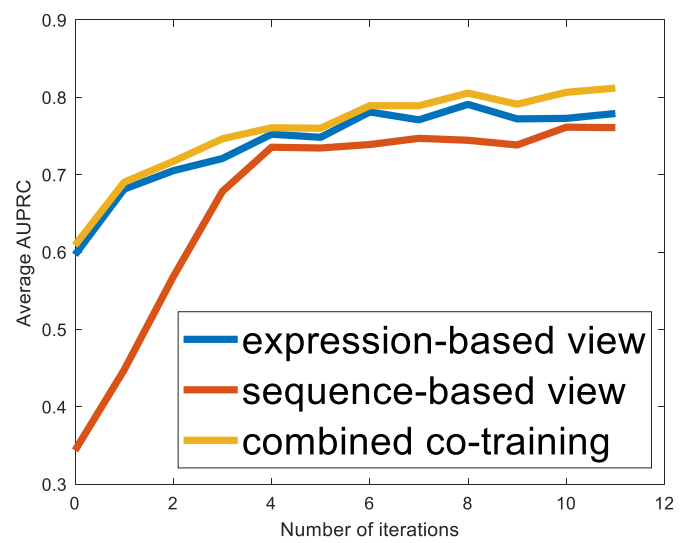

dme

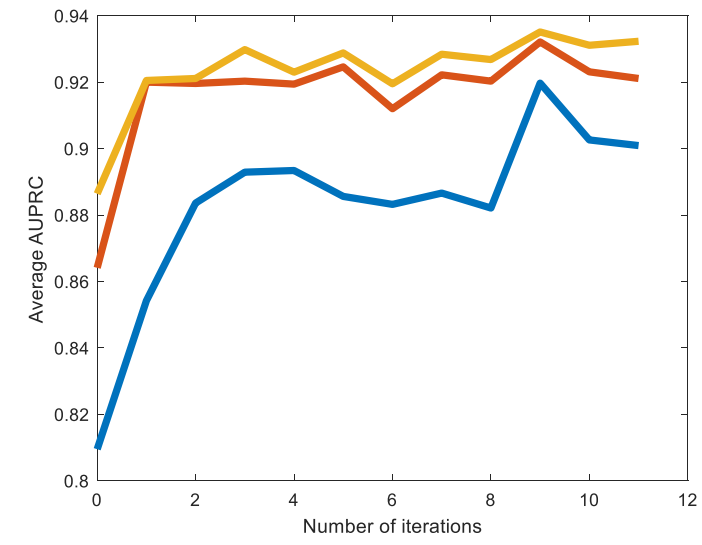

gga

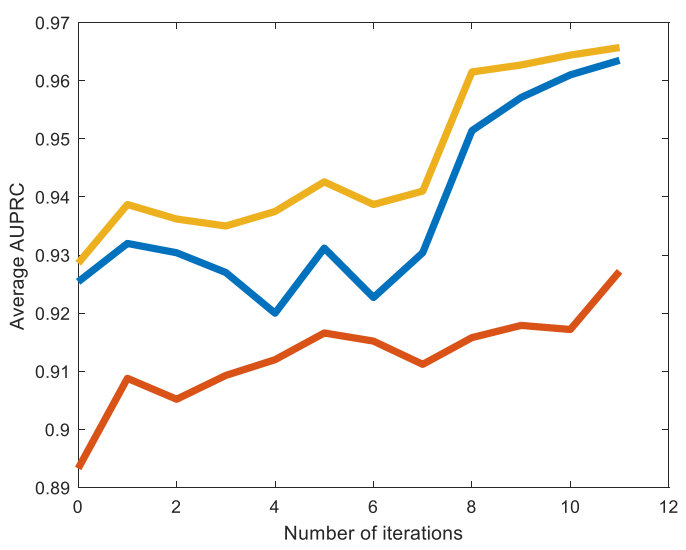

$m m u$

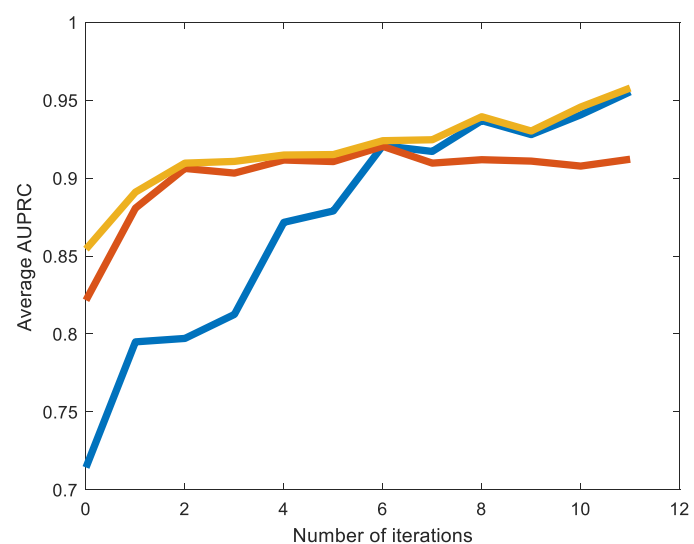

bta

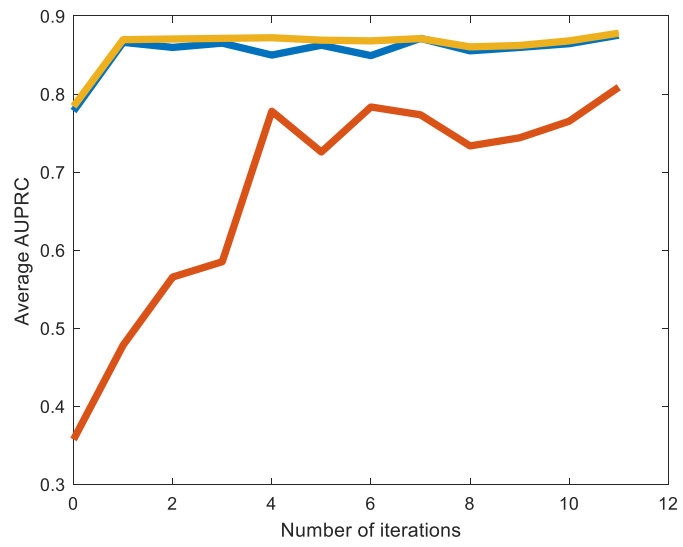

eca

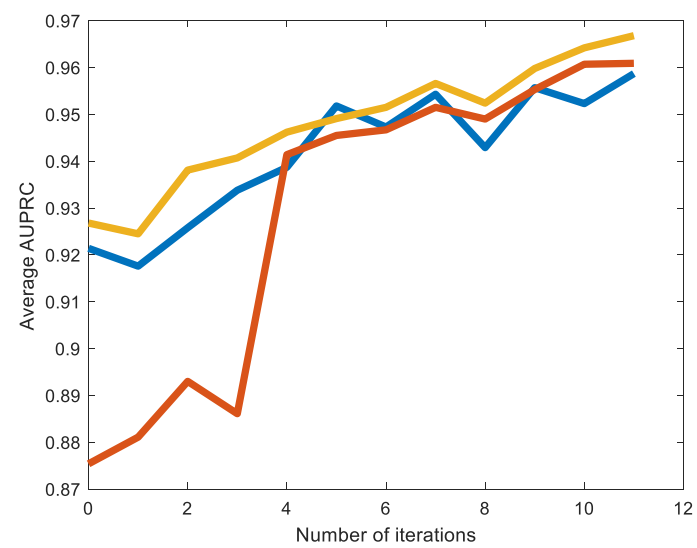

Figure 21. Performance of the sequence and expression-based classifiers and the combined co-training classifier at each iteration for all data sets 


\subsection{Discussion}

During our experiments, we compared co-training to self-training. In the self-training approach, although the most confidently predicted points are added to the training set at each iteration, we only make use of one view of the problem. By making this comparison to our multi-view co-training, we are observing the effectiveness of strengthening one view using the other. It can be observed that when only using one view to strengthen itself, the performance increase seems to plateau at a much earlier stage compared to co-training. Both expression and sequence-based classifiers trained using self-learning demonstrate increase in performance for a few iterations, but fail to continue this performance increase. This is due to the fact that the amount of evidence that one view can provide is limited, therefore limiting its ability in further strengthening the classifier. By comparing the results of self-training to our co-training approach in Table 11, the significance of using multiple views for training can be observed. From the results in Table 11, it can be observed that cotraining results in an expression-based classifier with an average AUPRC that is $3.91 \%$ higher than that of the self-training classifier over all the data sets. The resulting sequencebased classifier of co-training also demonstrates an increased average AUPRC of $6.94 \%$ over all data sets compared to the self-training classifier. These numbers alone suggest that there is value in taking advantage of the existence of multiple views for solving a problem.

In addition, from Table 11 it can be observed that in some cases, the self-training classifiers were unable to achieve substantial increases in performance. For example, the self-training expression-based classifiers for chicken and horse, there is a relatively small performance increase of $2.26 \%$ and $2.25 \%$, respectively. When looking at the average AUPRC for these experiments (Appendix A), it becomes apparent that they are already performing very well ( $0.946 \%$ and $0.942 \%$ respectively), leaving little room for improvement. The fact that the single-view classifiers could only increase performance by such a small margin, once again 
underlies the significance of using multiple views. This is because in these cases, the classifiers have already used most of the information available from that view. Adding any high confidence instances predicted by such a classifier to the training set will not further strengthen the view. At this point the classifier is simply adding instances with similar characteristics, therefore providing the classifier with redundant data. In contrast, using a different view for augmenting the training set could be highly valuable, as each added instance is both confidently predicted to be labeled correctly, and could provide new lines of evidence to the classifier. This results in the classifier continuously learning from the new evidence provided to it by the other view, and not only being provided with redundant information. As seen in Table 11, taking advantage of both views on the same problem results in almost twice the performance gain, going from $2.26 \%$ to $4.12 \%$ on the chicken experiment and from $2.25 \%$ to $4.02 \%$ on the horse experiment. These results highlight the significance of using multiple views (where applicable) for solving a pattern classification problem.

In the passive co-training approach implemented in this study, despite making use of both views of the problem similar to the co-training approach, we do not use the most confidently predicted instances. Instead, we choose expected positive and negative instances randomly and without knowing the model's confidence in their labelling. The results of this approach show a high degree of fluctuation during iterations, demonstrating performance increase in some and decrease in the others. This is due to the random selection of instances to add to the training set, which in some cases leads to a mislabeled instance being added to the training set, therefore decreasing the classifier's performance. When comparing the results of this passive approach to our co-training approach in Table 11 , it can be observed that strictly adding high confidence predictions has a significant effect on the classifier's performance. By doing so, co-training demonstrates a $10.97 \%$ higher average AUPRC over all the data sets for the expression-based classifier, and a 
$17.24 \%$ higher average AUPRC for the sequence-based classifier. In addition, from Table 11 it can be observed that for the expression-based classifier in the chicken and mouse data sets, passive learning actually decreased the performance of the original models by $0.22 \%$ and $0.83 \%$, respectively. This means that for these experiments, the addition of 22 instances resulted in weaker classifiers rather than strengthening them. This is due to the fact that a number of mislabeled points have been added to the original training sets for these species, causing the models to become weaker, since they are trained using incorrectly labelled data. These results underlie the importance of strictly adding high confidence instances to the training set, and that using multiple views alone is not sufficient for building a robust classifier.

The advantage of using multi-view co-training can be clearly seen in cases where one view initially performs much worse than the other. In Figure 18, it can be observed that in all of the data sets, one view clearly outperforms the other before the start of co-training. After 11 iterations, it is observed that the weaker view greatly improves in performance, and approaches the performance of the stronger view, even surpassing it in two data sets (mouse and horse). In other words, by using multiple views, one view can compensate the weak performance of another view. This demonstrates the advantage of co-training in using distinct views of a problem to strengthen each other, rather than having a weak view in classification. It must also be mentioned that during the different iterations of co-training, no misclassified instances were added to the training set of neither view. This means that in all cases, the top prediction of both views was a correct prediction, demonstrating the accuracy of the classifiers developed using co-training. The fact that all instances that are newly added to the training set are correctly predicted is evidence that this approach is successful. 
To further demonstrate the effectiveness of co-training, we combined the final view-based classifiers trained using co-training to create a combined co-training classifier. We compare the average area under precision recall curves in this combined classifier against each of the individual view-based classifiers in Figure 21 . The mispredictions that take place in individual views and lead to lower performance are commonly on the weaker predictions, which are the predictions around the decision boundary, with the probability of them belonging to each class being close to $50 \%$. When both views are combined, some of the instances that fall into the weak prediction zone for one of the views, are amongst the strong predictions in the other view. In the combined co-training classifier, we look at the prediction of both views for each instance. When we obtain each views' prediction score for that instance, we give the preference to the view with the higher confidence in prediction. By incorporating both views, we can take advantage of a greater number of strong predictions, therefore minimizing the number of weak predictions in our classifier and increasing performance.

The results from the combined co-training approach can also be compared to that of active learning in the previous chapter. It must be noted that in active learning, we are adding 22 annotated instances to the training set, whereas in co-training no annotations are performed. With this in mind, combined co-training outperforms active learning in 2 of the 6 data sets (fruit-fly and chicken) and yields comparable results on the other data sets. The fact that co-training demonstrates such robust performance without requiring any newly labeled samples is of great value. This is a significant factor in miRNA classification, as labeling is a time and cost expensive procedure, and being able to obtain such good results without requiring labeling is a great accomplishment. These results indicate the effectiveness of using multi-view co-training for miRNA classification. 


\subsection{Conclusion}

In this chapter, we design and develop a multi-view co-training approach for the classification of miRNA. By using a multi-view approach, we take advantage of both sequence and expression-based features to maximize miRNA classification ability. Using 11 iterations of co-training, the expression-based view of miRNA classification experiences a $15.81 \%$ increase on average over all data sets, compared to $11.90 \%$ for self-training and $4.84 \%$ for passive learning. The sequence-based classifier also experiences an increase of $46.47 \%$ across all species on average, where self-training and passive learning show $39.53 \%$ and $29.43 \%$ improvements, respectively. Finally, the developed sequence and expression-based classifiers are integrated into a final confidence-based combined cotraining classifier which shows improved performance compared to each of the individual views. Considering the fact that we are using an absolute minimum number of labeled instances for classification, these results demonstrate that multi-view co-training is a very effective approach for miRNA classification. This approach is expected to be particularly useful in cases where labelled training is scarce, such as for species that have been sequenced but not yet thoroughly analyzed or annotated through costly web-lab experiments. 


\section{Thesis Summary and Future Recommendations}

\subsection{Conclusions}

In this thesis, we explore two techniques of semi-supervised learning for the prediction of miRNA, active learning and multi-view co-training.

We here propose a novel active learning approach for the classification of miRNA, which takes advantage of both sequence- and expression-based features to maximize pattern classification ability. Our uncertainty-based active learning approach is compared to a variety of classifiers where no active learning is applied, and shows a consistent improvement in classification performance. Additionally, our active learning strategy created a classifier with improved performance compared to the state-of-the-art method miRDeep2. We demonstrate that our active learning approach is able to greatly improve classification performance using a small number of labeled instances, therefore suggesting that active learning is a suitable approach for miRNA classification.

We also design and develop a multi-view co-training approach for the classification of miRNA, with the goal of maximizing classification ability using both sequence- and expression-based features. Our approach is compared to self-training and passive learning over six data sets belonging to six distinct species, and demonstrates increased performance compared to all of them.

\subsection{Summary of contributions}

In this thesis, we perform a comprehensive comparison between active learning (both uncertainty query, certainty query), self-labelling, and passive learning strategies. We 
demonstrate that uncertainty-based query active learning can effectively reduce the number of labeling events required to obtain a high miRNA classification performance. As far as we have reviewed, this represents the first use of active learning applied to the problem of miRNA classification. When compared to the state-of-the-art miRDeep2 miRNA classification tool, our active learning approach proves superior over six distinct data sets. From our results, we can conclude that active learning is an efficient and effective approach for miRNA prediction in the face of limited training data.

Additionally, we have developed and applied a novel multi-view co-training approach for the classification of miRNA for the first time. In this approach, we take advantage of the existence of multiple views for miRNA classification, namely the sequence- and expressionbased views. Using this approach, we are able to make use of the multiple views to significantly reduce the number of labeled samples required for classification. We demonstrate that this co-training approach outperforms self-training and single view approaches. Therefore, by leveraging the two distinct views, we create more powerful classifiers without collecting any new labelled data. The strong performance of this method suggests that multi-view co-training is a suitable approach for miRNA prediction, especially in species that have not yet been well-annotated.

Lastly, this thesis has further explored the concept of an integrated feature set for miRNA prediction (i.e. the combination of expression- and sequence-based features in a single classifier). This idea was initially explored in the PhD thesis of Dr. Robert Peace [12]. Here, we have validated the method over additional species and have more thoroughly compared it to single-view classifiers with equivalent structure. These new contributions are reported in a manuscript currently under review by Scientific Reports, of which I am a co-author [68]. 


\subsection{Recommendations for future work}

A factor that can greatly contribute to any miRNA prediction approach, including our active leaning and co-training approaches, is developing stronger training data sets. This can be done by integrating data from multiple NGS experiments in order to create the positive and negative data sets. In our current work, we have considered only one data set for the development of the positive and negative data sets for each species. By combining multiple data sets, we can further increase prediction strength.

In addition, all classification results included in this paper are obtained using hold-out data sets, where labels were previously known and held back for experimentation. It would be ideal to be able to test our methods on truly unlabelled data sets. This would require wetlab experiments to determine the true class of the predictions. By doing so, we would be able to experimentally validate the novel predictions made by our proposed methods.

Lastly, active learning and multi-view co-training are not mutually exclusive. A combination of these two approaches should be investigated, where co-training is used to boost the performance of the classifier during each iteration of active learning. In this way, the classifier can benefit from self-labeling as much as possible before spending resources on new wet-lab experiments to collect more labelled data. 


\section{Appendix A}

Appendix A includes detailed results of active learning and co-training for different species.

\begin{tabular}{|c|c|c|c|c|}
$\begin{array}{c}\text { Active } \\
\text { learning } \\
\text { iteration }\end{array}$ & $\begin{array}{c}\text { mmu certainty- } \\
\text { based average } \\
\text { AUPRC }\end{array}$ & $\begin{array}{c}\text { mmu } \\
\text { uncertainty- } \\
\text { based average } \\
\text { AUPRC }\end{array}$ & $\begin{array}{c}\text { mmu certainty- } \\
\text { based average } \\
\text { AUROC }\end{array}$ & $\begin{array}{c}\text { mmu } \\
\text { uncertainty- } \\
\text { based average } \\
\text { AUROC }\end{array}$ \\
\hline $\mathbf{-}$ & 0.9134 & 0.9134 & 0.9552 & 0.9552 \\
\hline $\mathbf{1}$ & 0.8984 & 0.9607 & 0.9562 & 0.9847 \\
\hline $\mathbf{2}$ & 0.8922 & 0.9510 & 0.9582 & 0.9831 \\
\hline $\mathbf{3}$ & 0.9124 & 0.9630 & 0.9665 & 0.9878 \\
\hline $\mathbf{4}$ & 0.9355 & 0.9455 & 0.9736 & 0.9747 \\
\hline $\mathbf{5}$ & 0.9399 & 0.9608 & 0.9753 & 0.9788 \\
\hline $\mathbf{6}$ & 0.9148 & 0.9512 & 0.9657 & 0.9808 \\
\hline $\mathbf{7}$ & 0.9289 & 0.9501 & 0.9713 & 0.9777 \\
\hline $\mathbf{8}$ & 0.9184 & 0.9622 & 0.9676 & 0.9861 \\
\hline $\mathbf{9}$ & 0.9088 & 0.9595 & 0.9599 & 0.9831 \\
\hline $\mathbf{1 0}$ & 0.9204 & 0.9622 & 0.9677 & 0.9862 \\
\hline $\mathbf{1 1}$ & 0.9380 & 0.9716 & 0.9747 & 0.9936 \\
\hline
\end{tabular}

Table A1. Certainty and uncertainty-based active learning query strategy results for mouse data set

\begin{tabular}{|c|c|c|c|c|}
\hline $\begin{array}{c}\text { Active } \\
\text { learning } \\
\text { iteration }\end{array}$ & $\begin{array}{c}\text { dme certainty- } \\
\text { based average } \\
\text { AUPRC }\end{array}$ & $\begin{array}{c}\text { dme } \\
\text { uncertainty- } \\
\text { based average } \\
\text { AUPRC }\end{array}$ & $\begin{array}{c}\text { dme certainty- } \\
\text { based average } \\
\text { AUROC }\end{array}$ & $\begin{array}{c}\text { dme } \\
\text { uncertainty- } \\
\text { based average } \\
\text { AUROC }\end{array}$ \\
\hline $\mathbf{1}$ & 0.9110 & 0.9110 & 0.9426 & 0.9426 \\
\hline $\mathbf{1}$ & 0.9005 & 0.8874 & 0.9282 & 0.9115 \\
\hline $\mathbf{3}$ & 0.9039 & 0.8993 & 0.9306 & 0.9282 \\
\hline $\mathbf{4}$ & 0.8981 & 0.9059 & 0.9234 & 0.9330 \\
\hline $\mathbf{5}$ & 0.8966 & 0.9023 & 0.9258 & 0.9354 \\
\hline $\mathbf{6}$ & 0.8957 & 0.9054 & 0.9234 & 0.9330 \\
\hline $\mathbf{7}$ & 0.8992 & 0.9017 & 0.9234 & 0.9282 \\
\hline $\mathbf{8}$ & 0.9019 & 0.9119 & 0.9234 & 0.9426 \\
\hline $\mathbf{9}$ & 0.9037 & 0.9090 & 0.9330 & 0.9354 \\
\hline $\mathbf{1 0}$ & 0.8945 & 0.9190 & 0.9163 & 0.9522 \\
\hline $\mathbf{1 1}$ & 0.9004 & 0.9176 & 0.9234 & 0.9474 \\
\hline
\end{tabular}

Table A2. Certainty and uncertainty-based active learning query strategy results for fruit-fly data set 


\begin{tabular}{|c|c|c|c|c|}
\hline $\begin{array}{c}\text { Active } \\
\text { learning } \\
\text { iteration }\end{array}$ & $\begin{array}{c}\text { bta certainty- } \\
\text { based average } \\
\text { AUPRC }\end{array}$ & $\begin{array}{c}\text { bta } \\
\text { uncertainty- } \\
\text { based average } \\
\text { AUPRC }\end{array}$ & $\begin{array}{c}\text { bta certainty- } \\
\text { based average } \\
\text { AUROC }\end{array}$ & $\begin{array}{c}\text { bta } \\
\text { uncertainty- } \\
\text { based average } \\
\text { AUROC }\end{array}$ \\
\hline $\mathbf{-}$ & 0.8504 & 0.8504 & 0.9234 & 0.9234 \\
\hline $\mathbf{1}$ & 0.8142 & 0.8950 & 0.9209 & 0.9399 \\
\hline $\mathbf{2}$ & 0.8162 & 0.9158 & 0.9262 & 0.9480 \\
\hline $\mathbf{3}$ & 0.8476 & 0.9070 & 0.9404 & 0.9465 \\
\hline $\mathbf{4}$ & 0.8609 & 0.8507 & 0.9424 & 0.9076 \\
\hline $\mathbf{5}$ & 0.8790 & 0.8851 & 0.9491 & 0.9283 \\
\hline $\mathbf{6}$ & 0.8978 & 0.8723 & 0.9477 & 0.9213 \\
\hline $\mathbf{7}$ & 0.8769 & 0.8738 & 0.9463 & 0.9240 \\
\hline $\mathbf{8}$ & 0.8840 & 0.8744 & 0.9442 & 0.9338 \\
\hline $\mathbf{9}$ & 0.8889 & 0.9039 & 0.9345 & 0.9477 \\
\hline $\mathbf{1 0}$ & 0.9019 & 0.8992 & 0.9448 & 0.9428 \\
\hline $\mathbf{1 1}$ & 0.9206 & 0.9346 & 0.9516 & 0.9699 \\
\hline
\end{tabular}

Table A3. Certainty and uncertainty-based active learning query strategy results for cow data set

\begin{tabular}{|c|c|c|c|c|}
\hline $\begin{array}{c}\text { Active } \\
\text { learning } \\
\text { iteration }\end{array}$ & $\begin{array}{c}\text { gga certainty- } \\
\text { based average } \\
\text { AUPRC }\end{array}$ & $\begin{array}{c}\text { gga } \\
\text { uncertainty- } \\
\text { based average } \\
\text { AUPRC }\end{array}$ & $\begin{array}{c}\text { gga certainty- } \\
\text { based average } \\
\text { AUROC }\end{array}$ & $\begin{array}{c}\text { gga } \\
\text { uncertainty- } \\
\text { based average } \\
\text { AUROC }\end{array}$ \\
\hline $\mathbf{-}$ & 0.8909 & 0.8909 & 0.8681 & 0.8681 \\
\hline $\mathbf{1}$ & 0.9041 & 0.9108 & 0.8852 & 0.8938 \\
\hline $\mathbf{2}$ & 0.8950 & 0.9106 & 0.8657 & 0.8950 \\
\hline $\mathbf{3}$ & 0.9092 & 0.9222 & 0.8864 & 0.9072 \\
\hline $\mathbf{4}$ & 0.9061 & 0.9299 & 0.8730 & 0.9170 \\
\hline $\mathbf{5}$ & 0.8961 & 0.9382 & 0.8608 & 0.9292 \\
\hline $\mathbf{6}$ & 0.9002 & 0.9369 & 0.8608 & 0.9292 \\
\hline $\mathbf{7}$ & 0.9099 & 0.9349 & 0.8803 & 0.9280 \\
\hline $\mathbf{8}$ & 0.8987 & 0.9349 & 0.8730 & 0.9316 \\
\hline $\mathbf{9}$ & 0.9024 & 0.9383 & 0.8718 & 0.9402 \\
\hline $\mathbf{1 0}$ & 0.8971 & 0.9326 & 0.8718 & 0.9316 \\
\hline $\mathbf{1 1}$ & 0.9147 & 0.9444 & 0.8852 & 0.9463 \\
\hline
\end{tabular}

Table A4. Certainty and uncertainty-based active learning query strategy results for chicken data set 


\begin{tabular}{|c|c|c|c|c|}
\hline $\begin{array}{c}\text { Active } \\
\text { learning } \\
\text { iteration }\end{array}$ & $\begin{array}{c}\text { eca certainty- } \\
\text { based average } \\
\text { AUPRC }\end{array}$ & $\begin{array}{c}\text { eca } \\
\text { uncertainty- } \\
\text { based average } \\
\text { AUPRC }\end{array}$ & $\begin{array}{c}\text { eca certainty- } \\
\text { based average } \\
\text { AUROC }\end{array}$ & $\begin{array}{c}\text { eca } \\
\text { uncertainty- } \\
\text { based average } \\
\text { AUROC }\end{array}$ \\
\hline $\mathbf{-}$ & 0.9428 & 0.9428 & 0.9654 & 0.9654 \\
\hline $\mathbf{1}$ & 0.9515 & 0.9572 & 0.9641 & 0.9679 \\
\hline $\mathbf{2}$ & 0.9601 & 0.9624 & 0.9759 & 0.9781 \\
\hline $\mathbf{3}$ & 0.9620 & 0.9619 & 0.9762 & 0.9714 \\
\hline $\mathbf{4}$ & 0.9432 & 0.9686 & 0.9629 & 0.9813 \\
\hline $\mathbf{5}$ & 0.9598 & 0.9691 & 0.9730 & 0.9813 \\
\hline $\mathbf{6}$ & 0.9580 & 0.9712 & 0.9759 & 0.9835 \\
\hline $\mathbf{8}$ & 0.9605 & 0.9701 & 0.9749 & 0.9800 \\
\hline $\mathbf{9}$ & 0.9605 & 0.9710 & 0.9717 & 0.9816 \\
\hline $\mathbf{1 0}$ & 0.9580 & 0.9694 & 0.9676 & 0.9816 \\
\hline $\mathbf{1 1}$ & 0.9628 & 0.9696 & 0.9714 & 0.9810 \\
\hline $\mathbf{4}$ & 0.9680 & 0.9706 & 0.9778 & 0.9819 \\
\hline
\end{tabular}

Table A5. Certainty and uncertainty-based active learning query strategy results for horse data set

\begin{tabular}{|c|l|l|l|l|l|}
\hline Data set & $\begin{array}{l}\text { No learning } \\
\text { average } \\
\text { AUPRC }\end{array}$ & $\begin{array}{l}\text { Self-training } \\
\text { classifier's } \\
\text { average } \\
\text { AUPRC }\end{array}$ & $\begin{array}{l}\text { Passive } \\
\text { learning } \\
\text { classifier's } \\
\text { average } \\
\text { AUPRC }\end{array}$ & $\begin{array}{l}\text { Certainty- } \\
\text { based active } \\
\text { learning } \\
\text { classifier's } \\
\text { average } \\
\text { AUPRC }\end{array}$ & $\begin{array}{l}\text { Uncertainty- } \\
\text { based active } \\
\text { learning } \\
\text { classifier's } \\
\text { average } \\
\text { AUPRC }\end{array}$ \\
\hline hsa & $0.6964( \pm 0.02)$ & $0.7877( \pm 0.01)$ & $0.7886( \pm 0.02)$ & $0.7969( \pm 0.01)$ & $0.8752( \pm 0.01)$ \\
\hline $\boldsymbol{m m u}$ & $0.9134( \pm 0.02)$ & $0.9088( \pm 0.01)$ & $0.924( \pm 0.01)$ & $0.9380( \pm 0.00)$ & $0.9716( \pm 0.00)$ \\
\hline $\boldsymbol{d m e}$ & $0.9110( \pm 0.01)$ & $0.8957( \pm 0.01)$ & $0.9138( \pm 0.01)$ & $0.9170( \pm 0.01)$ & $0.9241( \pm 0.01)$ \\
\hline bta & $0.8504( \pm 0.02)$ & $0.8790( \pm 0.01)$ & $0.8665( \pm 0.02)$ & $0.9206( \pm 0.01)$ & $0.9346( \pm 0.01)$ \\
\hline gga & $0.8909( \pm 0.02)$ & $0.9026( \pm 0.01)$ & $0.8856( \pm 0.02)$ & $0.9147( \pm 0.00)$ & $0.9444( \pm 0.01)$ \\
\hline eca & $0.9428( \pm 0.01)$ & $0.9559( \pm 0.01)$ & $0.9538( \pm 0.01)$ & $0.9680( \pm 0.00)$ & $0.9706( \pm 0.00)$ \\
\hline $\begin{array}{c}\text { Avg. } \\
\text { increase }\end{array}$ & - & $+2.83 \%$ & $+2.86 \%$ & $+5.23 \%$ & $+8.72 \%$ \\
\hline
\end{tabular}

Table A6. Final classification results using certainty and uncertainty-based active learning strategies, passive training, self-learning and "no learning" for all six species. Standard deviation is shown in parentheses 


\begin{tabular}{|c|c|c|c|c|}
\hline $\begin{array}{c}\text { Co- } \\
\text { training } \\
\text { iteration }\end{array}$ & $\begin{array}{c}\text { mmu } \\
\text { expression- } \\
\text { based average }\end{array}$ & $\begin{array}{c}\text { mmu sequence- } \\
\text { based average } \\
\text { AUPRC }\end{array}$ & $\begin{array}{c}\text { mmu } \\
\text { expression- } \\
\text { based average } \\
\text { AUROC }\end{array}$ & $\begin{array}{c}\text { mmu sequence- } \\
\text { based average } \\
\text { AUROC }\end{array}$ \\
\hline $\mathbf{-}$ & 0.7143 & 0.8216 & 0.9143 & 0.9318 \\
\hline $\mathbf{1}$ & 0.7949 & 0.8807 & 0.9380 & 0.9437 \\
\hline $\mathbf{2}$ & 0.7971 & 0.9062 & 0.9395 & 0.9573 \\
\hline $\mathbf{3}$ & 0.8125 & 0.9032 & 0.9334 & 0.9613 \\
\hline $\mathbf{4}$ & 0.8716 & 0.9116 & 0.9485 & 0.9642 \\
\hline $\mathbf{6}$ & 0.8789 & 0.9106 & 0.9557 & 0.9623 \\
\hline $\mathbf{7}$ & 0.9208 & 0.9204 & 0.9721 & 0.9662 \\
\hline $\mathbf{8}$ & 0.9171 & 0.9096 & 0.9699 & 0.9588 \\
\hline $\mathbf{9}$ & 0.9369 & 0.9118 & 0.9779 & 0.9646 \\
\hline $\mathbf{1 0}$ & 0.9279 & 0.9109 & 0.9737 & 0.9610 \\
\hline $\mathbf{1 1}$ & 0.9406 & 0.9077 & 0.9761 & 0.9574 \\
\hline
\end{tabular}

Table A7. Co-training performance results for mouse data set

\begin{tabular}{|c|c|c|c|c|}
\hline $\begin{array}{c}\text { Co- } \\
\text { training } \\
\text { iteration }\end{array}$ & $\begin{array}{c}\text { dme expression- } \\
\text { expression-based } \\
\text { average AUPRC }\end{array}$ & $\begin{array}{c}\text { dme sequence- } \\
\text { sequence-based } \\
\text { average AUPRC }\end{array}$ & $\begin{array}{c}\text { dme expression- } \\
\text { based average } \\
\text { AUROC }\end{array}$ & $\begin{array}{c}\text { dme sequence- } \\
\text { based average } \\
\text { AUROC }\end{array}$ \\
\hline $\mathbf{-}$ & 0.8095 & 0.8641 & 0.7823 & 0.9043 \\
\hline $\mathbf{1}$ & 0.8542 & 0.9200 & 0.8565 & 0.9522 \\
\hline $\mathbf{2}$ & 0.8836 & 0.9196 & 0.8828 & 0.9522 \\
\hline $\mathbf{3}$ & 0.8929 & 0.9203 & 0.8995 & 0.9545 \\
\hline $\mathbf{4}$ & 0.8934 & 0.9194 & 0.9139 & 0.9545 \\
\hline $\mathbf{5}$ & 0.8856 & 0.9246 & 0.8780 & 0.9593 \\
\hline $\mathbf{6}$ & 0.8832 & 0.9120 & 0.8756 & 0.9378 \\
\hline $\mathbf{7}$ & 0.8866 & 0.9222 & 0.8876 & 0.9545 \\
\hline $\mathbf{8}$ & 0.8821 & 0.9203 & 0.8732 & 0.9522 \\
\hline $\mathbf{9}$ & 0.9197 & 0.9321 & 0.9498 & 0.9689 \\
\hline $\mathbf{1 0}$ & 0.9026 & 0.9231 & 0.9163 & 0.9569 \\
\hline $\mathbf{1 1}$ & 0.9009 & 0.9211 & 0.9115 & 0.9522 \\
\hline
\end{tabular}

Table A8. Co-training performance results for fruit-fly data set 


\begin{tabular}{|c|c|c|c|c|}
\hline $\begin{array}{c}\text { Co- } \\
\text { training } \\
\text { iteration }\end{array}$ & $\begin{array}{c}\text { bta expression- } \\
\text { based average } \\
\text { AUPRC }\end{array}$ & $\begin{array}{c}\text { bta sequence- } \\
\text { based average } \\
\text { AUPRC }\end{array}$ & $\begin{array}{c}\text { bta expression- } \\
\text { based average } \\
\text { AUROC }\end{array}$ & $\begin{array}{c}\text { bta sequence- } \\
\text { based average } \\
\text { AUROC }\end{array}$ \\
\hline- & 0.7782 & 0.3574 & 0.8814 & 0.5731 \\
\hline $\mathbf{1}$ & 0.8662 & 0.4782 & 0.9084 & 0.6874 \\
\hline $\mathbf{2}$ & 0.8597 & 0.5655 & 0.9210 & 0.8042 \\
\hline $\mathbf{3}$ & 0.8652 & 0.5851 & 0.9260 & 0.8052 \\
\hline $\mathbf{4}$ & 0.8499 & 0.7779 & 0.9145 & 0.8928 \\
\hline $\mathbf{5}$ & 0.8625 & 0.7257 & 0.9239 & 0.8829 \\
\hline $\mathbf{6}$ & 0.8492 & 0.7834 & 0.9230 & 0.8967 \\
\hline $\mathbf{7}$ & 0.8713 & 0.7734 & 0.9258 & 0.8966 \\
\hline $\mathbf{8}$ & 0.8553 & 0.7335 & 0.9223 & 0.8981 \\
\hline $\mathbf{9}$ & 0.8597 & 0.7439 & 0.9255 & 0.8818 \\
\hline $\mathbf{1 0}$ & 0.8645 & 0.7651 & 0.9154 & 0.9021 \\
\hline $\mathbf{1 1}$ & 0.8754 & 0.8087 & 0.9245 & 0.9161 \\
\hline
\end{tabular}

Table A9. Co-training performance results for cow data set

\begin{tabular}{|c|c|c|c|c|}
\hline $\begin{array}{c}\text { Co- } \\
\text { training } \\
\text { iteration }\end{array}$ & $\begin{array}{c}\text { gga expression- } \\
\text { based average } \\
\text { AUPRC }\end{array}$ & $\begin{array}{c}\text { gga sequence- } \\
\text { based average } \\
\text { AUPRC }\end{array}$ & $\begin{array}{c}\text { gga expression- } \\
\text { based average } \\
\text { AUROC }\end{array}$ & $\begin{array}{c}\text { gga sequence- } \\
\text { based average } \\
\text { AUROC }\end{array}$ \\
\hline $\mathbf{-}$ & 0.9254 & 0.8933 & 0.9353 & 0.8584 \\
\hline $\mathbf{1}$ & 0.9320 & 0.9088 & 0.9414 & 0.8889 \\
\hline $\mathbf{2}$ & 0.9304 & 0.9052 & 0.9341 & 0.8767 \\
\hline $\mathbf{3}$ & 0.9270 & 0.9093 & 0.9133 & 0.8913 \\
\hline $\mathbf{4}$ & 0.9200 & 0.9120 & 0.8974 & 0.8938 \\
\hline $\mathbf{5}$ & 0.9312 & 0.9166 & 0.9231 & 0.9011 \\
\hline $\mathbf{6}$ & 0.9227 & 0.9152 & 0.8987 & 0.8901 \\
\hline $\mathbf{7}$ & 0.9304 & 0.9112 & 0.9145 & 0.8840 \\
\hline $\mathbf{8}$ & 0.9514 & 0.9158 & 0.9585 & 0.8938 \\
\hline $\mathbf{9}$ & 0.9571 & 0.9179 & 0.9670 & 0.8950 \\
\hline $\mathbf{1 0}$ & 0.9610 & 0.9172 & 0.9731 & 0.8926 \\
\hline $\mathbf{1 1}$ & 0.9635 & 0.9272 & 0.9768 & 0.9121 \\
\hline
\end{tabular}

Table A10. Co-training performance results for chicken data set 


\begin{tabular}{|c|c|c|c|c|}
\hline $\begin{array}{c}\text { Co- } \\
\text { training } \\
\text { iteration }\end{array}$ & $\begin{array}{c}\text { eca expression- } \\
\text { based average } \\
\text { AUPRC }\end{array}$ & $\begin{array}{c}\text { eca sequence- } \\
\text { based average } \\
\text { AUPRC }\end{array}$ & $\begin{array}{c}\text { eca expression- } \\
\text { based average } \\
\text { AUROC }\end{array}$ & $\begin{array}{c}\text { eca sequence- } \\
\text { based average } \\
\text { AUROC }\end{array}$ \\
\hline- & 0.9214 & 0.8754 & 0.9324 & 0.8508 \\
\hline $\mathbf{1}$ & 0.9176 & 0.8811 & 0.9311 & 0.8571 \\
\hline $\mathbf{2}$ & 0.9258 & 0.8930 & 0.9340 & 0.8740 \\
\hline $\mathbf{3}$ & 0.9338 & 0.8861 & 0.9273 & 0.8552 \\
\hline $\mathbf{4}$ & 0.9387 & 0.9414 & 0.9194 & 0.9283 \\
\hline $\mathbf{5}$ & 0.9518 & 0.9455 & 0.9467 & 0.9378 \\
\hline $\mathbf{6}$ & 0.9473 & 0.9467 & 0.9384 & 0.9419 \\
\hline $\mathbf{7}$ & 0.9543 & 0.9515 & 0.9597 & 0.9441 \\
\hline $\mathbf{8}$ & 0.9429 & 0.9490 & 0.9444 & 0.9451 \\
\hline $\mathbf{9}$ & 0.9557 & 0.9553 & 0.9556 & 0.9590 \\
\hline $\mathbf{1 0}$ & 0.9523 & 0.9607 & 0.9498 & 0.9530 \\
\hline $\mathbf{1 1}$ & 0.9587 & 0.9609 & 0.9571 & 0.9606 \\
\hline
\end{tabular}

Table A11. Co-training performance results for horse data set

\begin{tabular}{|c|c|c|c|c|c|c|c|c|}
\hline \multirow{2}{*}{$\begin{array}{l}\text { Data } \\
\text { set }\end{array}$} & \multicolumn{4}{|c|}{ Expression-based classifier's average AUPRC } & \multicolumn{4}{|c|}{ Sequence-based classifier's average AUPRC } \\
\hline & No learning & $\begin{array}{l}\text { Passive } \\
\text { learning }\end{array}$ & Self-training & Co-training & $\begin{array}{l}\text { No } \\
\text { learning }\end{array}$ & $\begin{array}{l}\text { Passive } \\
\text { learning }\end{array}$ & Self-training & Co-training \\
\hline hsa & $0.597( \pm 0.03)$ & $0.672( \pm 0.02)$ & $0.770( \pm 0.02)$ & $0.779( \pm 0.01)$ & $0.344( \pm 3.2)$ & $0.616( \pm 0.03)$ & $0.720( \pm 0.02)$ & $0.761( \pm 0.01)$ \\
\hline$m m u$ & $0.714( \pm 0.01)$ & $0.708( \pm 0.02)$ & $0.883( \pm 0.01)$ & $0.955( \pm 0.01)$ & $0.822( \pm 1.2)$ & $0.887( \pm 0.02)$ & $0.895( \pm 0.01)$ & $0.912( \pm 0.01)$ \\
\hline dme & $0.810( \pm 0.02)$ & $0.883( \pm 0.01)$ & $0.886( \pm 0.01)$ & $0.901( \pm 0.01)$ & $0.864( \pm 1.8)$ & $0.909( \pm 0.02)$ & $0.912( \pm 0.01)$ & $0.921( \pm 0.01)$ \\
\hline$b t a$ & $0.778( \pm 0.02)$ & $0.827( \pm 0.02)$ & $0.815( \pm 0.02)$ & $0.865( \pm 0.01)$ & $0.357( \pm 2.8)$ & $0.654( \pm 0.02)$ & $0.732( \pm 0.02)$ & $0.809( \pm 0.01)$ \\
\hline$g g a$ & $0.925( \pm 0.01)$ & $0.923( \pm 0.02)$ & $0.946( \pm 0.01)$ & $0.964( \pm 0.01)$ & $0.893( \pm 1.6)$ & $0.894( \pm 0.02)$ & $0.911( \pm 0.02)$ & $0.927( \pm 0.01)$ \\
\hline eca & $0.921( \pm 0.01)$ & $0.941( \pm 0.01)$ & $0.942( \pm 0.01)$ & $0.958( \pm 0.01)$ & $0.875( \pm 1.6)$ & $0.884( \pm 0.02)$ & $0.932( \pm 0.01)$ & $0.961( \pm 0.01)$ \\
\hline $\begin{array}{l}\text { Avg } \\
\text { incr. }\end{array}$ & - & $+4.84 \%$ & $+11.90 \%$ & $+15.81 \%$ & - & $+29.43 \%$ & $+39.53 \%$ & $+46.47 \%$ \\
\hline
\end{tabular}

Table A12. Final classification results using co-training, single-view (self-training), passive learning and no learning for expression and sequence-based views. Standard deviation is shown in parentheses 


\section{References}

[1] Y. W. Iwasaki et al., "Global microRNA elevation by inducible Exportin 5 regulates cell cycle entry," RNA, vol. 19, no. 4, pp. 490-497, 2013.

[2] A. La Torre, S. Georgi, and T. A. Reh, "Conserved microRNA pathway regulates developmental timing of retinal neurogenesis," Proceedings of the National Academy of Sciences, vol. 110, no. 26, pp. E2362-E2370, 2013.

[3] Z. Ren and V. R. Ambros, "Caenorhabditis elegans microRNAs of the let-7 family act in innate immune response circuits and confer robust developmental timing against pathogen stress," Proceedings of the National Academy of Sciences, p. 201422858, 2015.

[4] T. Otto et al., "Cell cycle-targeting microRNAs promote differentiation by enforcing cell-cycle exit," Proceedings of the National Academy of Sciences, vol. 114, no. 40, pp. 10660-10665, 2017.

[5] M. Isik, T. K. Blackwell, and E. Berezikov, "MicroRNA mir-34 provides robustness to environmental stress response via the DAF-16 network in C. elegans," Scientific Reports, vol. 6, p. 36766, 2016.

[6] D. Andolina et al., "Effects of lack of microRNA-34 on the neural circuitry underlying the stress response and anxiety," Neuropharmacology, vol. 107, pp. 305-316, 2016.

[7] S. L. Hollins and M. J. Cairns, "MicroRNA: Small RNA mediators of the brains genomic response to environmental stress," Progress in Neurobiology, vol. 143, pp. 61-81, 2016.

[8] A. Kozomara and S. Griffiths-Jones, "miRBase: annotating high confidence microRNAs using deep sequencing data," Nucleic Acids Research, vol. 42, no. D1, pp. D68-D73, 2013.

[9] R. J. Peace and J. R. Green, "Computational Sequence- and NGS-based MicroRNA Prediction," in Biomedical Signal Processing in Big Data: CRC Press, 2016.

[10] R. J. Peace, K. K. Biggar, K. B. Storey, and J. R. Green, "A framework for improving microRNA prediction in non-human genomes," Nucleic Acids Research, vol. 43, no. 20, pp. e138-e138, 2015.

[11] K. C. Miranda et al., "A pattern-based method for the identification of MicroRNA binding sites and their corresponding heteroduplexes," Cell, vol. 126, no. 6, pp. 1203-1217, 2006.

[12] R. Peace, "MicroRNA Prediction for Unannotated Genome-Wide and Transcriptomic Experiments," Carleton University Ottawa, 2016.

[13] H. S. Kim et al., "MicroRNA-31 functions as a tumor suppressor by regulating cell cycle and epithelial-mesenchymal transition regulatory proteins in liver cancer," Oncotarget, vol. 6, no. 10, p. 8089, 2015.

[14] Q. Luo et al., "Tumor-suppressive microRNA-195-5p regulates cell growth and inhibits cell cycle by targeting cyclin dependent kinase 8 in colon cancer," American Journal of Translational Research, vol. 8, no. 5, p. 2088, 2016.

[15] O. F. Karatas et al., "miR-33a is a tumor suppressor microRNA that is decreased in prostate cancer," Oncotarget, vol. 8, no. 36, p. 60243, 2017.

[16] S. Sugita et al., "Mp65-10 the tumor suppressor microrna-223 targets Wdr62 directly in bladder cancer," The Journal of Urology, vol. 199, no. 4, p. e862, 2018.

[17] S. C. Forster, M. D. Tate, and P. J. Hertzog, "MicroRNA as type I interferon-regulated transcripts and modulators of the innate immune response," Frontiers in Immunology, vol. 6, p. 334, 2015. 
[18] H. Hadj-Moussa and K. B. Storey, "Micromanaging freeze tolerance: the biogenesis and regulation of neuroprotective microRNAs in frozen brains," Cellular and Molecular Life Sciences, pp. 1-13, 2018.

[19] C.-W. Wu, K. K. Biggar, and K. B. Storey, "Dehydration mediated microRNA response in the African clawed frog Xenopus laevis," Gene, vol. 529, no. 2, pp. 269-275, 2013.

[20] M. H. Sohel, "Extracellular/circulating microRNAs: release mechanisms, functions and challenges," Achievements in the Life Sciences, vol. 10, no. 2, pp. 175-186, 2016.

[21] L.-A. MacFarlane and P. R Murphy, "MicroRNA: biogenesis, function and role in cancer," Current Genomics, vol. 11, no. 7, pp. 537-561, 2010.

[22] A. Válóczi, C. Hornyik, N. Varga, J. Burgyán, S. Kauppinen, and Z. Havelda, "Sensitive and specific detection of microRNAs by northern blot analysis using LNAmodified oligonucleotide probes," Nucleic Acids Research, vol. 32, no. 22, pp. e175e175, 2004.

[23] C. Chen et al., "Real-time quantification of microRNAs by stem-loop RT-PCR," Nucleic Acids Research, vol. 33, no. 20, pp. e179-e179, 2005.

[24] W. Li and K. Ruan, "MicroRNA detection by microarray," Analytical and Bioanalytical chemistry, vol. 394, no. 4, pp. 1117-1124, 2009.

[25] F. Li et al., "Carbon nanotube-based label-free electrochemical biosensor for sensitive detection of miRNA-24," Biosensors and Bioelectronics, vol. 54, pp. 158$164,2014$.

[26] D. W. Hwang, I. C. Song, D. S. Lee, and S. Kim, "Smart magnetic fluorescent nanoparticle imaging probes to monitor microRNAs," Small, vol. 6, no. 1, pp. 81-88, 2010.

[27] C. Li, Z. Li, H. Jia, and J. Yan, "One-step ultrasensitive detection of microRNAs with loop-mediated isothermal amplification (LAMP)," Chemical Communications, vol. 47, no. 9, pp. 2595-2597, 2011.

[28] T. Tian, J. Wang, and X. Zhou, "A review: microRNA detection methods," Organic \& Biomolecular Chemistry, vol. 13, no. 8, pp. 2226-2238, 2015.

[29] R. O. Duda, P. E. Hart, and D. G. Stork, Pattern classification. John Wiley \& Sons, 2012.

[30] D. H. Wolpert, "The lack of a priori distinctions between learning algorithms," Neural Computation, vol. 8, no. 7, pp. 1341-1390, 1996.

[31] R. Quinlan, "Programs for machine learning morgan kaufmann publishers inc," San Francisco, USA, 1993.

[32] L. Breiman, "Random forests," Machine learning, vol. 45, no. 1, pp. 5-32, 2001.

[33] V. Vapnik, The Nature of Statistical Learning Theory. Springer science \& business media, 2013.

[34] J. A. Swets, "ROC analysis applied to the evaluation of medical imaging techniques," Investigative Radiolology, v14 i2, pp. 09-121, 1979.

[35] A. J. Joshi, F. Porikli, and N. Papanikolopoulos, "Multi-class active learning for image classification" IEEE Conference on Computer Vision and Pattern Recognition, 2009, pp. 2372-2379: IEEE.

[36] Y. Baram, R. E. Yaniv, and K. Luz, "Online choice of active learning algorithms," Journal of Machine Learning Research, vol. 5, no. Mar, pp. 255-291, 2004.

[37] D. D. Lewis and J. Catlett, "Heterogeneous uncertainty sampling for supervised learning," in Machine Learning Proceedings 1994: Elsevier, 1994, pp. 148-156.

[38] D. D. Lewis and W. A. Gale, "A sequential algorithm for training text classifiers," in Proceedings of the 17th annual international ACM SIGIR conference on Research and Development in Information Retrieval, 1994, pp. 3-12: Springer-Verlag New York, Inc.

[39] T. P. Mohamed, J. G. Carbonell, and M. K. Ganapathiraju, "Active learning for human protein-protein interaction prediction," BMC Bioinformatics, vol. 11, no. 1, p. S57, 2010. 
[40] M. K. Warmuth, G. Rätsch, M. Mathieson, J. Liao, and C. Lemmen, "Active learning in the drug discovery process," in Advances in Neural Information Processing Systems, 2002, pp. 1449-1456.

[41] J. Zhu, H. Wang, T. Yao, and B. K. Tsou, "Active learning with sampling by uncertainty and density for word sense disambiguation and text classification," in Proceedings of the 22nd International Conference on Computational LinguisticsVolume 1, 2008, pp. 1137-1144: Association for Computational Linguistics.

[42] G. Schohn and D. Cohn, "Less is more: Active learning with support vector machines," in ICML, 2000, pp. 839-846: Citeseer.

[43] A. Vlachos, "A stopping criterion for active learning," Computer Speech \& Language, vol. 22, no. 3, pp. 295-312, 2008.

[44] A. Blum and T. Mitchell, "Combining labeled and unlabeled data with co-training," in Proceedings of the eleventh annual conference on Computational Learning Theory, 1998, pp. 92-100: ACM.

[45] E. Berezikov, E. Cuppen, and R. H. Plasterk, "Approaches to microRNA discovery," Nature Genetics, vol. 38, p. S2, 2006.

[46] L. P. Lim et al., "The microRNAs of Caenorhabditis elegans," Genes \& Development, vol. 17, no. 8, pp. 991-1008, 2003.

[47] L. P. Lim, M. E. Glasner, S. Yekta, C. B. Burge, and D. P. Bartel, "Vertebrate microRNA genes," Science, vol. 299, no. 5612, pp. 1540-1540, 2003.

[48] E. C. Lai, P. Tomancak, R. W. Williams, and G. M. Rubin, "Computational identification of Drosophila microRNA genes," Genome Biology, vol. 4, no. 7, p. R42, 2003.

[49] Y. Grad et al., "Computational and experimental identification of C. elegans microRNAs," Molecular Cell, vol. 11, no. 5, pp. 1253-1263, 2003.

[50] T. Dezulian, M. Remmert, J. F. Palatnik, D. Weigel, and D. H. Huson, "Identification of plant microRNA homologs," Bioinformatics, vol. 22, no. 3, pp. 359-360, 2005.

[51] A. Adai et al., "Computational prediction of miRNAs in Arabidopsis thaliana," Genome Research, vol. 15, no. 1, pp. 78-91, 2005.

[52] M. W. Jones-Rhoades and D. P. Bartel, "Computational identification of plant microRNAs and their targets, including a stress-induced miRNA," Molecular Cell, vol. 14, no. 6, pp. 787-799, 2004.

[53] C. P. D. C. Gomes, J.-H. Cho, L. E. Hood, O. L. Franco, R. W. D. Pereira, and K. Wang, "A review of computational tools in microRNA discovery," Frontiers in Genetics, vol. 4, p. 81, 2013.

[54] V. Balatti, Y. Pekarky, and C. M. Croce, "Role of microRNA in chronic lymphocytic leukemia onset and progression," Journal of Hematology \& Oncology, vol. 8, no. 1, p. $12,2015$.

[55] C. Xue, F. Li, T. He, G.-P. Liu, Y. Li, and X. Zhang, "Classification of real and pseudo microRNA precursors using local structure-sequence features and support vector machine," BMC Bioinformatics, vol. 6, no. 1, p. 310, 2005.

[56] P. Jiang, H. Wu, W. Wang, W. Ma, X. Sun, and Z. Lu, "MiPred: classification of real and pseudo microRNA precursors using random forest prediction model with combined features," Nucleic Acids Research, vol. 35, no. suppl_2, pp. W339-W344, 2007.

[57] S. Kadri, V. Hinman, and P. V. Benos, "HHMMiR: efficient de novo prediction of microRNAs using hierarchical hidden Markov models," BMC Bioinformatics, vol. 10, no. 1, p. S35, 2009.

[58] R. Batuwita and V. Palade, "microPred: effective classification of pre-miRNAs for human miRNA gene prediction," Bioinformatics, vol. 25, no. 8, pp. 989-995, 2009.

[59] J. Xiao et al., "Identification of microRNA precursors based on random forest with network-level representation method of stem-loop structure," BMC Bioinformatics, vol. 12 , no. 1 , p. $165,2011$. 
[60] M. R. Friedländer, S. D. Mackowiak, N. Li, W. Chen, and N. Rajewsky, "miRDeep2 accurately identifies known and hundreds of novel microRNA genes in seven animal clades," Nucleic Acids Research, vol. 40, no. 1, pp. 37-52, 2011.

[61] M. R. Friedländer et al., "Discovering microRNAs from deep sequencing data using miRDeep," Nature Biotechnology, vol. 26, no. 4, p. 407, 2008.

[62] B. Langmead, C. Trapnell, M. Pop, and S. L. Salzberg, "Ultrafast and memoryefficient alignment of short DNA sequences to the human genome," Genome biology, vol. 10, no. 3, p. R25, 2009.

[63] M. Hackenberg, M. Sturm, D. Langenberger, J. M. Falcon-Perez, and A. M. Aransay, "miRanalyzer: a microRNA detection and analysis tool for next-generation sequencing experiments," Nucleic Acids Research, vol. 37, no. suppl_2, pp. W68W76, 2009.

[64] A. Gudyś, M. W. Szcześniak, M. Sikora, and I. Makałowska, "HuntMi: an efficient and taxon-specific approach in pre-miRNA identification," BMC Bioinformatics, vol. 14, no. 1, p. 83, 2013.

[65] Q. Zou, Y. Mao, L. Hu, Y. Wu, and Z. Ji, "miRClassify: an advanced web server for miRNA family classification and annotation," Computers in Biology and Medicine, vol. 45, pp. 157-160, 2014.

[66] A. Kozomara and S. Griffiths-Jones, "miRBase: integrating microRNA annotation and deep-sequencing data," Nucleic Acids Research, vol. 39, no. suppl_1, pp. D152D157, 2010.

[67] C. Paicu et al., "miRCat2: accurate prediction of plant and animal microRNAs from next-generation sequencing datasets," Bioinformatics, vol. 33, no. 16, pp. 24462454, 2017.

[68] R.J. Peace, M. Sheikh Hassani, J.R. Green, "miPIE : NGS-based Prediction of miRNA Using Integrated Evidence," Scientific Reports, vol. Under review, 2018.

[69] N. Du et al., "Gene Co-Adaboost: a semi-supervised approach for classifying gene expression data," in Proceedings of the 2nd ACM Conference on Bioinformatics, Computational Biology and Biomedicine, 2011, pp. 531-535: ACM.

[70] A. Sokolov and A. Ben-Hur, "Multi-view prediction of protein function," in Proceedings of the 2nd ACM Conference on Bioinformatics, Computational Biology and Biomedicine, 2011, pp. 135-142: ACM.

[71] J. Kim and H. Shin, "Breast cancer survivability prediction using labeled, unlabeled, and pseudo-labeled patient data," Journal of the American Medical Informatics Association, vol. 20, no. 4, pp. 613-618, 2013.

[72] Y.-Y. Xu, F. Yang, Y. Zhang, and H.-B. Shen, "Bioimaging-based detection of mislocalized proteins in human cancers by semi-supervised learning," Bioinformatics, vol. 31, no. 7, pp. 1111-1119, 2014.

[73] R. Ibrahim, N. A. Yousri, M. A. Ismail, and N. M. El-Makky, "miRNA and gene expression based cancer classification using self-learning and co-training approaches,", 2013 IEEE International Conference on Bioinformatics and Biomedicine (BIBM), 2013, pp. 495-498: IEEE.

[74] M. J. Jones and P. Viola, "Robust real-time object detection," in Workshop on Statistical and Computational Theories of Vision, 2001, vol. 266, p. 56.

[75] Z.-H. Zhou and M. Li, "Tri-training: Exploiting unlabeled data using three classifiers," IEEE Transactions on Knowledge and Data Engineering, vol. 17, no. 11, pp. 15291541, 2005.

[76] Y.-C. Gong and C.-L. Chen, "Semi-supervised method for gene expression data classification with Gaussian fields and harmonic functions," 19th International Conference on Pattern Recognition, Tampa, USA, 2008. pp. 1-4: IEEE.

[77] D. Roqueiro, M. J. Witteveen, V. Anttila, G. M. Terwindt, A. M. van den Maagdenberg, and $\mathrm{K}$. Borgwardt, "In silico phenotyping via co-training for improved phenotype prediction from genotype," Bioinformatics, vol. 31, no. 12, pp. i303-i310, 2015. 
[78] L. Breiman, "Bagging predictors," Machine Learning, vol. 24, no. 2, pp. 123-140, 1996.

[79] G. Forman, "Incremental machine learning to reduce biochemistry lab costs in the search for drug discovery," in Proceedings of the 2nd International Conference on Data Mining in Bioinformatics, 2002, pp. 33-36: Springer-Verlag.

[80] Y. Liu, "Active learning with support vector machine applied to gene expression data for cancer classification," Journal of Chemical Information and Computer Sciences, vol. 44, no. 6, pp. 1936-1941, 2004.

[81] S. Doyle, J. Monaco, M. Feldman, J. Tomaszewski, and A. Madabhushi, "A class balanced active learning scheme that accounts for minority class problems: Applications to histopathology," in OPTIMHisE Workshop (MICCAI), 2009, pp. 19-30.

[82] R. K. Padmanabhan et al., "An active learning approach for rapid characterization of endothelial cells in human tumors," PLoS One, vol. 9, no. 3, p. e90495, 2014.

[83] D. A. Pertusi, M. E. Moura, J. G. Jeffryes, S. Prabhu, B. W. Biggs, and K. E. Tyo, "Predicting novel substrates for enzymes with minimal experimental effort with active learning," Metabolic Engineering, vol. 44, pp. 171-181, 2017.

[84] W. Xiong, L. Xie, S. Zhou, and J. Guan, "Active learning for protein function prediction in protein-protein interaction networks," Neurocomputing, vol. 145, pp. 44-52, 2014.

[85] F. O. Iyuke, J. R. Green, and W. G. Willmore, "Active Learning for the Prediction of Asparagine/Aspartate Hydroxylation Sites on Proteins," IASTED, Pitsburg , 2011.

[86] D. Reker, P. Schneider, G. Schneider, and J. Brown, "Active learning for computational chemogenomics," Future Medicinal Chemistry, vol. 9, no. 4, pp. 381402, 2017.

[87] T. Barrett et al., "NCBI GEO: archive for functional genomics data sets-update," Nucleic Acids Research, vol. 41, no. D1, pp. D991-D995, 2012.

[88] J. Casper et al., "The UCSC Genome Browser database: 2018 update," Nucleic Acids Research, vol. 46, no. D1, pp. D762-D769, 2017.

[89] B. L. Aken et al., "Ensembl 2017," Nucleic Acids Research, vol. 45, no. D1, pp. D635D642, 2016.

[90] I. Kalvari et al., "Rfam 13.0: shifting to a genome-centric resource for non-coding RNA families," Nucleic acids research, vol. 46, no. D1, pp. D335-D342, 2017.

[91] Y. Huang, B. Niu, Y. Gao, L. Fu, and W. Li, "CD-HIT Suite: a web server for clustering and comparing biological sequences," Bioinformatics, vol. 26, no. 5, pp. 680-682, 2010.

[92] S. Lertampaiporn, C. Thammarongtham, C. Nukoolkit, B. Kaewkamnerdpong, and M. Ruengjitchatchawalya, "Heterogeneous ensemble approach with discriminative features and modified-SMOTE bagging for pre-miRNA classification," Nucleic Acids Research, vol. 41, no. 1, pp. e21-e21, 2012.

[93] F. Pedregosa et al., "Scikit-learn: Machine learning in Python," Journal of Machine Learning Research, vol. 12, no. Oct, pp. 2825-2830, 2011.

[94] M. Hall, E. Frank, G. Holmes, B. Pfahringer, P. Reutemann, and I. H. Witten, "The WEKA data mining software: an update," ACM SIGKDD Explorations Newsletter, vol. 11, no. 1, pp. 10-18, 2009.

[95] V. Williamson, A. Kim, B. Xie, G. O. McMichael, Y. Gao, and V. Vladimirov, "Detecting miRNAs in deep-sequencing data: a software performance comparison and evaluation," Briefings in Bioinformatics, vol. 14, no. 1, pp. 36-45, 2012.

[96] D. Leshkowitz, S. Horn-Saban, Y. Parmet, and E. Feldmesser, "Differences in microRNA detection levels are technology and sequence dependent," RNA, vol. 19, no. 4, pp. 527-538, 2013.

[97] U. Brefeld and T. Scheffer, "Semi-supervised learning for structured output variables," in Proceedings of the 23rd International Conference on Machine learning, 2006, pp. 145-152: ACM. 
[98] S. Clark, J. R. Curran, and M. Osborne, "Bootstrapping POS taggers using unlabelled data," in Proceedings of the seventh conference on Natural Language Learning at HLT-NAACL 2003-Volume 4, 2003, pp. 49-55: Association for Computational Linguistics.

[99] K. Nigam and R. Ghani, "Analyzing the effectiveness and applicability of co-training," in Proceedings of the ninth international conference on Information and Knowledge Management, 2000, pp. 86-93: ACM.

[100] R. Mihalcea, "Co-training and self-training for word sense disambiguation," in Proceedings of the Eighth Conference on Computational Natural Language Learning (CONLL-2004) at HLT-NAACL 2004, 2004. 